\title{
TOWARDS DIFFERENTIAL CALCULUS IN STRATIFIED GROUPS
}

\author{
VALENTINO MAGNANI \\ (Received 26 June 2010; accepted 16 October 2011; first published online 17 June 2013) \\ Communicated by M. Cowling
}

\begin{abstract}
We study graded group-valued continuously differentiable mappings defined on stratified groups, where differentiability is understood with respect to the group structure. We characterize these mappings by a system of nonlinear first-order PDEs, establishing a quantitative estimate for their difference quotient. This provides us with a mean value estimate that allows us to prove both the inverse mapping theorem and the implicit function theorem. The latter theorem also relies on the fact that the differential admits a proper factorization of the domain into a suitable inner semidirect product. When this splitting property of the differential holds in the target group, then the inverse mapping theorem leads us to the rank theorem. Both implicit function theorem and rank theorem naturally introduce the classes of image sets and level sets. For commutative groups, these two classes of sets coincide and correspond to the usual submanifolds. In noncommutative groups, we have two distinct classes of intrinsic submanifolds. They constitute the so-called intrinsic graphs, that are defined with respect to the algebraic splitting and everywhere possess a unique metric tangent cone equipped with a natural group structure.
\end{abstract}

2010 Mathematics subject classification: primary 22E30; secondary 26B10, $26 \mathrm{~B} 12$.

Keywords and phrases: stratified groups, implicit function theorem, mean value inequality, rank theorem, intrinsic submanifolds.

\section{Introduction}

The relationship between the geometry of stratified groups and several branches of Mathematics, such as PDEs, Differential Geometry, Complex Analysis, Control Theory and Geometric Measure Theory, has known an increasing interest in the last decades.

The initial purpose of this work was the study of a general framework to properly state and prove an implicit function theorem for mappings between stratified groups. Here the first aspect to clarify is the notion of differentiability, that can be naturally

(c) 2013 Australian Mathematical Publishing Association Inc. 1446-7887/2013 \$16.00 
stated taking into account the homogeneous structure of the group [24]. We will always refer to this notion of differentiability, if not otherwise stated.

An implicit function theorem for differentiable mappings requires a thorough study of these mappings. Although the notion of differentiability defined by group dilations and group translations reminds of the classical one, see Definition 2.6, this notion is indeed highly nonlinear. In fact, our first purpose is that of studying this nonlinearity, searching for its analytic characterization in terms of PDEs.

This study in particular leads us to both the implicit function theorem and the rank theorem below stated. From the development of these tools, we naturally meet the notion of regular set modeled on a couple of groups. A discussion on this project can be found in the first version of this work [20].

The notions we use in the following will be precisely introduced in Section 2. Let $\mathbb{G}$ and $\mathbb{M}$ denote a stratified group and a graded group, respectively. The corresponding Lie algebras $\mathcal{G}$ of $\mathbb{G}$ and $\mathcal{M}$ of $\mathbb{M}$ are decomposed into the direct sums of layers $V_{i}$ and $W_{i}$, respectively. The mappings $\pi_{j}$ indicate the canonical projection onto the $j$ th layer of a graded algebra. The symbol $D$ denotes the differential. In the next theorem, we will consider the differential of the mappings $F_{j}$, that take values in the subspaces $W_{j}$. In fact, these linear spaces can be also regarded as commutative stratified groups.

THeorem 1.1 (Characterization of differentiable mappings). Let $\Omega \subset \mathbb{G}$ be an open set and let $f: \Omega \longrightarrow \mathbb{M}$. Then the following statements are equivalent:

- $\quad f$ is continuously differentiable;

- if $f(x)=\exp \circ F(x)=\exp \sum_{j=1}^{v} F_{j}(x)$, then $F_{j}: \Omega \longrightarrow W_{j}$, equal to $\pi_{j} \circ F$, are continuously differentiable and the formulas

$$
\begin{gathered}
D f(x)(\exp Y)=\exp \left(Y F_{1}(x)\right) \\
D F_{j}(x)(h)=\sum_{n=2}^{v} \frac{(-1)^{n}}{n !} \pi_{j}\left([F(x), D F(x)(h)]_{n-1}\right)
\end{gathered}
$$

hold for every $Y \in V_{1}$, every $j=2, \ldots, v$ and every $h \in \mathbb{G}$;

- $\quad f$ is continuously h-differentiable contact.

The integer $v$ denotes the step of $\mathbb{M}$ and $D f$ denotes the differential of $f$.

Let us point out that $h$-differentiability corresponds to the classical differentiability restricted to horizontal directions. This notion coincides with differentiability if and only if the target is commutative and it becomes a weaker notion as soon as the target is noncommutative. Theorem 1.1 shows that horizontal differentiability joined with the contact property characterizes differentiability. Thus, by Theorem 1.1 we can study differentiable mappings through the following system of first-order 
nonlinear PDEs

$$
\left\{\begin{array}{l}
X_{1} F_{j}=\sum_{n=2}^{v} \frac{(-1)^{n}}{n !} \pi_{j}\left(\left[F, X_{1} F\right]_{n-1}\right) \\
X_{2} F_{j}=\sum_{n=2}^{v} \frac{(-1)^{n}}{n !} \pi_{j}\left(\left[F, X_{2} F\right]_{n-1}\right) \\
\vdots \quad \vdots \quad \vdots \quad \vdots \quad \vdots \quad \vdots \quad \vdots \\
X_{m} F_{j}=\sum_{n=2}^{v} \frac{(-1)^{n}}{n !} \pi_{j}\left(\left[F, X_{m} F\right]_{n-1}\right),
\end{array}\right.
$$

where $j=2, \ldots, v$ and $\left(X_{1}, \ldots, X_{m}\right)$ is a basis of the first layer $V_{1}$ of $\mathcal{G}$. In fact, all the previous systems of first-order PDEs (1.3) are equivalent to the equations of (1.2) and exactly characterize the contact property of $f$. The nonlinearity of these equations comes from both the iterated commutators $[X, Y]_{n-1}=$ $[X,[X, \ldots,[X, Y]] \ldots]$ introduced in Definition 3.3 and from the polynomial form of $X_{l} F_{j}$ with respect to $F_{1}, \ldots, F_{j-1}, X_{l} F_{1}, \ldots, X_{l} F_{j-1}$. This can be easily seen by the recursion structure of (1.3). The equations of (1.3), namely the 'contact equations', have been used in [21] to characterize Lipschitz mappings and study Lipschitz extensions problems. This recursion structure has been also used to establish an 'algebraic regularity theorem' in the study of regularity of 1-quasiconformal mappings, see [2].

Let us point out that in our study we only require the codomain of the mapping to be graded and not necessarily stratified. Graded groups in general need not be connected by rectifiable curves, according to Examples 2.1 and 2.2. In particular, our Corollary 4.7 extends the almost everywhere differentiability of Lipschitz mappings, proved in [24], to the case where the target need not be connected by rectifiable curves.

If one assumes a priori the $C^{1}$ smoothness of $f$, then the characterization of contact mappings by differentiability is proved in [29]. The intriguing feature of Theorem 1.1, along with its technical difficulty, stems from the fact that we do not assume any 'extrinsic' regularity of $f$. Here it is worth emphasizing the existence of continuously differentiable functions that are not $C^{1}$ in the Euclidean sense and in this sense they are not differentiable on sets of positive measure [18].

The crucial point in proving Theorem 1.1 is the estimate of the following difference quotient of a horizontal curve $\Gamma=\exp \circ \gamma$. We define $\gamma_{i}=\pi_{i} \circ \gamma$ and observe that the exponential of the element

$$
\delta_{1 / h}\left(-h \dot{\gamma}_{1}(t) \odot(-\gamma(t) \odot \gamma(t+h))\right)
$$

is equal to $\delta_{1 / h}\left(\left(\exp \left(\dot{\gamma}_{1}(t) h\right)\right)^{-1} \Gamma(t)^{-1} \Gamma(t+h)\right)$, where $\delta_{h}$ here denotes the dilation in the target group and $\odot$ is the group operation on the Lie algebra, which is provided by the Baker-Campbell-Hausdorff formula. Taking into account this fact, we can state the following key estimate of Theorem 4.6:

$$
\left\|\delta_{1 / h}\left(-h \dot{\gamma}_{1}(t) \odot(-\gamma(t)) \odot \gamma(t+h)\right)\right\| \leq \Upsilon\left(L_{t}\right) \mathcal{A}_{t}^{h}\left(\dot{\gamma}_{1}-\dot{\gamma}_{1}(t)\right),
$$


where $\mathcal{A}_{t}^{h}\left(\dot{\gamma}_{1}-\dot{\gamma}_{1}(t)\right)$ denotes the 'sup-average' of $s \rightarrow \dot{\gamma}_{1}(s)-\dot{\gamma}_{1}(t)$. This leads us to the uniform convergence of the difference quotient along horizontal directions, eventually proving the differentiability. We also apply (1.4) to the restriction of the difference quotient of $f$ along a fixed family of piecewise horizontal lines that span an open set. These curves are collected by the mappings $P^{s}$, introduced in Definition 4.8, and have an associated geometric constant $c(\mathbb{G}, d)$, given in Definition 4.10. In this case, we arrive at the proof of (1.6), where $\omega_{\bar{\Omega}_{2}, d_{H} F_{1}}$ denotes the modulus of continuity of the horizontal differential $d_{H} F_{1}$ and $F_{1}$ is the vector representing the horizontal components of $f$. The Lie algebras will be always assumed to be equipped with a norm, denoted by $\|\cdot\|$. This norm in $\mathcal{G}$ naturally yields a left invariant subFinsler norm on $\mathbb{G}$ and then allows for defining an operator norm $\left\|d_{H} F_{1}(x)\right\|$ for $d_{H} F_{1}(x): H_{x} \mathbb{G} \longrightarrow W_{1}$. With this notation, we have the following theorem.

THeOrem 1.2 (Mean value estimate). Let $\Omega \subset \mathbb{G}$ be open and consider a continuously differentiable mapping $f: \Omega \longrightarrow \mathbb{M}$. Let $\Omega_{1}, \Omega_{2} \subset \mathbb{G}$ be open subsets such that

$$
\left\{x \in \mathbb{G} \mid d\left(x, \Omega_{1}\right) \leq c(\mathbb{G}, d) N \operatorname{diam}\left(\Omega_{1}\right)\right\} \subset \Omega_{2},
$$

where $\Omega_{2}$ is compactly contained in $\Omega$. Then there exist a constant $C$, only depending on $\mathbb{G}, \max _{x \in \bar{\Omega}_{2}}\left\|d_{H} F_{1}(x)\right\|$ and on $\omega_{\bar{\Omega}_{2}, d_{H} F_{1}}\left(\operatorname{diam}\left(\Omega_{2}\right)\right)$, defined in (4.11), such that

$$
\frac{\rho\left(f(x)^{-1} f(y), D f(x)\left(x^{-1} y\right)\right)}{d(x, y)} \leq C\left[\omega_{\bar{\Omega}_{2}, d_{H} F_{1}}(N c(\mathbb{G}, d) d(x, y))\right]^{1 / \iota^{2}}
$$

for every $x, y \in \bar{\Omega}_{1}$, with $x \neq y$. The integer ı denotes the step of $\mathbb{G}$.

Let us point out that the condition (1.5) has only a technical nature. In fact, it is used only to make sure that our fixed family of piecewise horizontal lines, connecting all points of $\Omega_{1}$ and along which we apply (1.4), are all contained in $\Omega_{2}$.

There are a number of technical difficulties that appear in the proof of the estimate (1.6). First, differentiable mappings in general need not be $C^{1}$ smooth in the classical sense. Second, working by single components does not suffice, since this would lead us to estimates of the Euclidean norm of the difference quotient. Here the problem stems from the fact that the Euclidean norm is not equivalent to the homogeneous norm and the latter is required in the notion of differentiability. We wish to stress that better families of horizontal curves may lead to better estimates. In fact, the exponent $1 / \iota^{2}$ in (1.6) is not sharp and certainly can be improved in many single cases. On the other hand, our interest in this estimate is that of establishing a uniform convergence of the difference quotient of continuously differentiable mappings. This key fact leads us to an important theorem of Calculus.

Theorem 1.3 (Inverse mapping theorem). Let $\Omega \subset \mathbb{G}$ be open, let $f: \Omega \longrightarrow \mathbb{G}$ be continuously differentiable and let $D f(\bar{x}): \mathbb{G} \longrightarrow \mathbb{G}$ be invertible, with $\bar{x} \in \Omega$. Then there exists a neighborhood $U$ of $\bar{x}$ such that the restriction $f_{\mid U}$ has an inverse mapping $g$ that is also differentiable and for every $y \in f(U)$ we have $D g(y)=D f(g(y))^{-1}$. 
Another important result of Calculus is the classical implicit function theorem. We observe that the implicit mapping is classically defined by decomposing the space into a product of two linear subspaces, naturally given by the tangent space to the level set and one of its complementary subspaces. In stratified groups, subspaces are replaced by homogeneous subgroups that are Lie subgroups closed under dilations. However, extending the previous scheme to this setting does not work, since the first subgroup is automatically given by the kernel of the differential of the defining mapping, but one could not always find any 'complementary subgroup'.

In view of this difficulty, we replace complementary subspaces by complementary subgroups and introduce special classes of $h$-epimorphisms and $h$-monomorphisms, see Section 7 for more details. If $N$ and $H$ are complementary subgroups of $\mathbb{G}$ and $D_{p, s} \subset \mathbb{G}$ is a closed ball of center $p$ and radius $s>0$, we use the convenient notation $D_{p, s}^{N}=D_{p, s} \cap N$ and $D_{p, s}^{H}=D_{p, s} \cap H$. We are now in the position to state one of the central results of this paper.

Theorem 1.4 (Implicit function theorem). Let $\Omega \subset \mathbb{G}$ be an open set and let $f: \Omega \longrightarrow$ $\mathbb{M}$ be continuously differentiable, where $\bar{x} \in \Omega$ and the differential $D f(\bar{x}): \mathbb{G} \longrightarrow \mathbb{M}$ is an h-epimorphism. Let $N$ be the kernel of $D f(\bar{x})$ and let $H$ be a complementary subgroup. Then there exist $r, s>0$, with $D_{\bar{n}, r}^{N} D_{\bar{h}, s}^{H} \subset \Omega$, along with a unique mapping $\varphi: D_{\bar{n}, r}^{N} \longrightarrow D_{\bar{h}, s}^{H}$, such that

$$
f^{-1}(f(\bar{x})) \cap D_{\bar{n}, r}^{N} D_{\bar{h}, s}^{H}=\left\{n \varphi(n) \mid n \in D_{\bar{n}, r}^{N}\right\} .
$$

Furthermore, there exists a constant $\kappa>0$ such that the Lipschitz-type estimate

$$
d\left(\varphi(n), \varphi\left(n^{\prime}\right)\right) \leq \kappa d\left(\varphi\left(n^{\prime}\right)^{-1} n^{-1} n^{\prime} \varphi\left(n^{\prime}\right)\right)
$$

continuous with respect to the metrics $d$ in $D_{\bar{h}, r}^{H}$ and $\|\cdot\|$ in $D_{\bar{n}, s}^{N}$.

It is worth mentioning that the standard application of the contraction mapping principle to prove the classical implicit function theorem does not work for groups. In fact, to use this argument, where we assume $\bar{x}=e$ and $f(e)=e$, one should consider the mapping $F(h)=\left(L^{-1} \circ f(n h)\right)^{-1} h$, where $L$ is the restriction of the differential $D f(e)$ to the complementary subgroup $H$. In the classical case, one can show that $F$ is a contraction for every $n$ sufficiently close to $e$, and hence obtaining the fixed point $h(n)$. In stratified groups this argument fails, since the pointwise product of differentiable mappings need not be differentiable, for noncommutative targets.

To overcome this point, we show that the mapping $F_{n}(h)=f(n h)$ is uniformly bi-Lipschitz with respect to $n$ and has constant nonvanishing topological degree as $n$ varies in a compact neighborhood of the unit element of $N$. This gives existence and uniqueness of the implicit mapping. Here the crucial point resides in the proof of the bi-Lipschitz continuity of $F_{n}$, that relies on both the mean value estimate of Theorem 1.2 and on the proper inner semidirect factorization of $\mathbb{G}$.

To prove the rank theorem, the algebraic splitting is required to hold in the target. Then we refer to our special class of injective $h$-homomorphisms, that we call 
$h$-monomorphisms, see Section 7. These mappings yield the natural splitting of the target and this property is precisely a characterization of these $h$-homomorphisms, according to Proposition 7.11.

Theorem 1.5 (Rank theorem). Let $f: \Omega \longrightarrow \mathbb{M}$ be a continuously differentiable mapping, where $\Omega$ is an open subset of $\mathbb{G}$. Let $\bar{x} \in \Omega$ and let $D f(\bar{x}): \mathbb{G} \longrightarrow \mathbb{M}$ be the differential. Let us assume that $D f(\bar{x})$ is an h-monomorphism with image $H$ and let $N$ be a normal complementary subgroup. Let $p: \mathbb{M} \longrightarrow H$ be the associated canonical projection. Then there exist neighborhoods $V \subset \Omega$ of $\bar{x}$ and $W \subset H$ of $p(f(\bar{x}))$ along with mappings $\varphi: W \longrightarrow N, J: H \longrightarrow \mathbb{G}$ and $\Psi: f(V) \longrightarrow \mathbb{M}$ such that we have

$$
f(V)=\{h \varphi(h) \mid h \in W\} \quad \text { and } \quad \Psi \circ f \circ J_{\mid V^{\prime}}=I_{\mid V^{\prime}},
$$

where $I: H \hookrightarrow \mathbb{M}$ is the restriction of the identity mapping $I d_{\mathbb{M}}$ to $H \subset \mathbb{M}$, the open subset $V^{\prime} \subset H$ is equal to $J^{-1}(V)$ and $J$ is equal to $(p \circ D f(\bar{x}))^{-1}$. Furthermore, setting $F=\exp ^{-1} \circ \varphi$, there exists $C>0$ such that for every $h, h^{\prime} \in W$,

$$
\left\|F(h)-F\left(h^{\prime}\right)\right\| \leq C d\left(h, h^{\prime}\right) .
$$

The proof of this result strongly differs from that of the implicit function theorem. Here the key observation is that the projection $p: \mathbb{M} \longrightarrow H$ is an $h$-homomorphism, and hence the composition $p \circ f: \Omega \longrightarrow H$ has invertible differential at $\bar{x}$. This allows us to apply the inverse mapping theorem stated in Theorem 1.3.

Implicit function theorem and rank theorem are the standard tools to define regular sets of the Euclidean space and, more generally, differentiable manifolds. Analogously, in an obvious way Theorem 1.4 and Theorem 1.5 define subsets that are expected to possess some intrinsic regularity. These are the $(\mathbb{G}, \mathbb{M})$-regular sets, which we distinguish into those contained in $\mathbb{G}$, which are suitable level sets, and those contained in $\mathbb{M}$, which are suitable image sets, see Section 10 for more details. By estimate (9.8), which immediately follows from $(1.8)$, ( $\mathbb{G}, \mathbb{M})$-regular sets of $\mathbb{G}$ can be locally parametrized by $1 / \iota$-Hölder mappings. In the case of $\left(\mathbb{H}^{1}, \mathbb{R}\right)$-regular sets of $\mathbb{H}^{1}$, where $\iota=2$, Kirchheim and Serra Cassano have proved that the Hölder exponent $1 / 2$ cannot be improved. In the same work [13], the authors also provide an interesting example of $\left(\mathbb{H}^{1}, \mathbb{R}\right)$-regular set of $\mathbb{H}^{1}$ of Euclidean Hausdorff dimension $5 / 2$. A simple consequence of both the rank theorem and the implicit function theorem is the following corollary.

\section{Corollary 1.6. Every $(\mathbb{G}, \mathbb{M})$-regular set is locally an intrinsic graph.}

In general stratified groups, $(\mathbb{G}, \mathbb{M})$-regular sets of $\mathbb{G}$ have been considered in [19], without assuming that the differential of the defining mapping is an $h$-epimorphism. New examples of $(\mathbb{G}, \mathbb{M})$-regular sets can be found in Theorem 12.6, where all possible regular sets of the six-dimensional complexified Heisenberg group $\mathbb{H}_{2}^{1}$ are provided. To establish this classification, we find all possible factorizations of $\mathbb{H}_{2}^{1}$ into an inner semidirect product of two complementary subgroups, then we apply both Theorem 1.4 and Theorem 12.1. The latter theorem shows that $\left(\mathbb{R}^{k}, \mathbb{M}\right)$-submanifolds of $\mathbb{M}$ coincide 
with $k$-dimensional $C^{1}$ horizontal submanifolds of $\mathbb{M}$, namely, those submanifolds that are tangent to the horizontal distribution of $\mathbb{M}$. Another consequence of Theorem 12.1, joined with Theorem 1.5, is that horizontal submanifolds are intrinsic graphs.

From the metric viewpoint, estimate (1.10) shows a stronger regularity of image sets with respect to level sets, where the 'nonlinear estimate' (1.8) holds. However, both level sets and image sets everywhere possess metric tangent cones equipped with a group structure.

THEOREM 1.7 (Intrinsic blow-up). Under the hypotheses of Theorem 1.4, we consider the set $S=f^{-1}(f(\bar{x}))$. Then for every $R>0$,

$$
D_{R} \cap \delta_{1 / \lambda}\left((\bar{x})^{-1} S\right) \longrightarrow D_{R} \cap N \quad \text { as } \lambda \rightarrow 0^{+}
$$

with respect to the Hausdorff convergence of sets, and hence $\operatorname{Tan}(S, \bar{x})=N$.

Under the hypotheses of Theorem 1.5, setting $S=f(V)$, for every $R>0$,

$$
D_{R} \cap \delta_{1 / \lambda}\left((\bar{x})^{-1} S\right) \longrightarrow D_{R} \cap H \quad \text { as } \lambda \rightarrow 0^{+}
$$

with respect to the Hausdorff convergence of sets, and hence $\operatorname{Tan}(S, \bar{x})=H$.

As a consequence of this theorem, we notice another strong difference between level sets and image sets in general. In fact, all homogeneous tangent cones to a ( $\mathbb{G}, \mathbb{M})$ regular set of $\mathbb{M}$ are clearly $h$-isomorphic to $\mathbb{G}$ and, in particular, they have all the same Hausdorff dimension. Furthermore, by the area formula of [15], their Hausdorff dimension coincides with that of $\mathbb{G}$ and there is an integral formula for their Hausdorff measure. On the other side, homogeneous tangent cones to a $(\mathbb{G}, \mathbb{M})$-regular set of $\mathbb{G}$ are not necessarily $h$-isomorphic to each other, see Example 10.7, but they are all normal subgroups of $\mathbb{G}$. In Corollary 10.6 we show that all homogeneous tangent cones $\operatorname{Tan}(S, \bar{x})$ to a $(\mathbb{G}, \mathbb{M})$-regular set of $\mathbb{G}$ have the same Hausdorff dimension and

$$
\operatorname{Tan}(S, \bar{x})=\mathcal{H}-\operatorname{dim}(\mathbb{G})-\mathcal{H}-\operatorname{dim}(\mathbb{M}) .
$$

This suggests that the Hausdorff dimension of a $(\mathbb{G}, \mathbb{M})$-regular set of $\mathbb{G}$ is expected to be that of the homogeneous tangent cones. This is indeed the case for $\left(\mathbb{H}^{n}, \mathbb{R}^{k}\right)$-regular sets of $\mathbb{H}^{n}$, with $1 \leq k \leq n$, for which Franchi, Serapioni and Serra Cassano have also established an area-type formula to compute their spherical Hausdorff measure [7]. Then the natural question is that of studying the validity of an area-type formula for any $(\mathbb{G}, \mathbb{M})$ )-regular set of $\mathbb{G}$.

The previous results suggest that the existence of many $(\mathbb{G}, \mathbb{M})$-regular sets is related to the existence of large families of $h$-homomorphisms from $\mathbb{G}$ to $\mathbb{M}$, along with suitable factorizing properties. In Section 11, we address novel applications of both the rank theorem and the implicit function theorem, considering some special couples of groups where all injective $h$-homomorphisms are $h$-monomorphisms and all surjective $h$-homomorphisms are $h$-epimorphisms. All of these facts suggest how new geometric models could be studied through the development of sufficiently deep algebraic tools. 


\section{Preliminaries and definitions}

All Lie groups we consider in this paper are real, connected, simply connected and finite dimensional. A graded group is a Lie group $\mathbb{G}$, whose Lie algebra $\mathcal{G}$ can be written as the direct sum of subspaces $V_{i}$, called layers, such that $\left[V_{i}, V_{j}\right] \subset V_{i+j}$ and $\mathcal{G}=V_{1} \oplus \cdots \oplus V_{\iota}$. The integer $\iota$ is the step of nilpotence of $\mathbb{G}[4]$. A graded group $\mathbb{G}$ is stratified if its layers satisfy the stronger condition $\left[V_{i}, V_{j}\right]=V_{i+j}$.

The grading of $\mathcal{G}$ allows us to introduce a one-parameter group of Lie algebra automorphisms $\delta_{r}: \mathcal{G} \longrightarrow \mathcal{G}$, defined as $\delta_{r}(X)=r^{i}$ if $X \in V_{i}$, where $r>0$. These mappings are called dilations. Taking into account that the exponential mapping $\exp : \mathcal{G} \longrightarrow \mathbb{G}$ is a diffeomorphism for simply connected nilpotent Lie groups, we can read dilations in the group $\mathbb{G}$ through the mapping exp and maintain the same notation. Recall from [28, Theorem 2.14.3] that the differential of the exponential mapping is given by the following formula

$$
d \exp (X)=\operatorname{Id}-\sum_{n=2}^{\infty} \frac{(-1)^{n}}{n !} \operatorname{ad}(X)^{n-1} .
$$

The group operation can be read in the algebra as follows

$$
X \odot Y=\sum_{j=1}^{\iota} c_{n}(X, Y)
$$

where $c_{1}(X, Y)=X+Y$ and the addends $c_{n}$ are given by induction through the BakerCampbell-Hausdorff formula

$$
\begin{aligned}
& (n+1) c_{n+1}(X, Y)=\frac{1}{2}\left[X-Y, c_{n}(X, Y)\right] \\
& \quad+\sum_{\substack{p \geq 1 \\
2 p \leq n}} K_{2 p} \sum_{\substack{k_{1}, \ldots, k_{2 p}>0 \\
k_{1}+\cdots+k_{2 p}=n}}\left[c_{k_{1}}(X, Y),\left[\ldots,\left[c_{k_{2 p}}(X, Y), X+Y\right]\right] \ldots\right],
\end{aligned}
$$

see [28, Lemma 2.15.3]. Analyzing (2.3), one easily notices that

$$
c_{n}(\lambda X, \lambda Y)=\lambda^{n} c_{n}(X, Y)
$$

for every $X, Y \in \mathcal{G}$ and $\lambda \in \mathbb{R}$. These formulas will be important in the next section.

The metric structure of a graded group is given by a continuous, left invariant distance $d: \mathbb{G} \times \mathbb{G} \longrightarrow \mathbb{R}$ such that $d\left(\delta_{r} x, \delta_{r} y\right)=r d(x, y)$ for every $x, y \in \mathbb{G}$ and $r>0$. Every distance satisfying these properties is a homogeneous distance.

The Carnot-Carathéodory distance is an important example of homogeneous distance that can be defined in stratified groups, since they satisfy the Lie bracket generating condition, see for instance [8].

Notice that graded groups may not satisfy this condition. On the other hand, according to Example 2.2, when a graded group is contained in a stratified group, 
the restriction of the Carnot-Carathéodory distance to the graded subgroup provides an example of homogeneous distance. In general, it is possible to construct homogeneous distances in every graded group $[6,10]$.

EXAMPLE 2.1. Let $\mathbb{R}^{n} \times \mathbb{R}$ be equipped with the sum of vectors as commutative group operation and define the parabolic distance $|(x, t)|=|x|+\sqrt{|t|}$ and dilations $\delta_{r}(x, t)=$ $\left(r x, r^{2} t\right)$. Then $\mathbb{M}$ is a 2-step graded group, that is neither stratified nor connected by rectifiable curves.

Example 2.2. Let $\mathbb{H}^{1}$ denote the Heisenberg group with its Heisenberg algebra $\mathfrak{h}^{1}=\operatorname{span}\{X, Y, Z\}$, where $[X, Y]=Z$. Then the 'vertical' subgroup $\exp (\operatorname{span}\{X, Z\})$ equipped with the restriction of the Carnot-Carathéodory distance is a graded group, but it is not stratified.

We denote by $e$ the unit element and to simplify notation we set $d(x)=d(x, e)$. Notice that left invariance and symmetry of $d$ imply the equality $d\left(x^{-1}\right)=d(x)$. An open ball of center $x$ and radius $r$ with respect to a homogeneous distance will be denoted by $B_{x, r}$. The corresponding closed ball will be denoted by $D_{x, r}$.

\section{1. $h$-homomorphisms and notions of differentiability.}

Definition 2.3 ( $h$-homomorphism). Let $\mathbb{G}$ and $\mathbb{M}$ be graded groups with dilations $\delta_{r}^{\mathbb{G}}$ and $\delta_{r}^{\mathbb{M}}$, respectively. We say that a group homomorphism $L: \mathbb{G} \longrightarrow \mathbb{M}$ such that $L\left(\delta_{r}^{\mathbb{G}} x\right)=\delta_{r}^{\mathbb{M}} L(x)$ for every $x \in \mathbb{G}$ and $r>0$ is a homogeneous homomorphism, in short $h$-homomorphism. Invertible $h$-homomorphisms will be called $h$-isomorphisms.

REMARK. Analogous terminology will be used for the corresponding Lie algebra homomorphisms of graded algebras that commute with dilations.

Remark. We introduce ' $h$-epimorphisms' and ' $h$-monomorphisms' to indicate surjective and injective $h$-homomorphisms, respectively. We notice that a surjective linear mapping of vector spaces is characterized by the existence of a right inverse that is also linear. Analogously, injective linear mappings are characterized by the existence of a linear left inverse mapping. The analogous characterization for either surjective or injective algebra homomorphisms does not work, as we will see in the next example.

Example 2.4. Let $l: \mathfrak{h}^{1} \longrightarrow \mathbb{R}^{2}$ be a surjective $h$-homomorphism. Clearly, $l(\mathfrak{v})=\mathbb{R}^{2}$ and $l(\mathfrak{z})=\{0\}$, where $\mathfrak{h}^{1}=\mathfrak{v} \oplus \mathfrak{z}$. We show that there is no right inverse that is also an $h$-homomorphism. Assume by contradiction that there exists an $h$-homomorphism $\tau: \mathbb{R}^{2} \longrightarrow \mathfrak{h}^{1}$ that is a right inverse. Then the property $l \circ \tau=\operatorname{Id}_{\mathbb{R}^{2}}$ and the fact that $\tau$ is an $h$-homomorphism imply that $\tau\left(\mathbb{R}^{2}\right)$ is a two-dimensional homogeneous subalgebra of $\mathfrak{h}^{1}$. Clearly, $\tau\left(\mathbb{R}^{2}\right)$ cannot intersect $\operatorname{ker} l=\mathfrak{\jmath}$, but this conflicts with Example 7.7, where we show that any two-dimensional homogeneous subalgebra of $\mathfrak{h}^{1}$ contains $\mathfrak{z}$.

As a consequence of the previous example, in the category of graded algebras and $h$-homomorphisms, requiring the existence of a right inverse homomorphism is a stronger condition than surjectivity. This motivates the following definition. 
Definition 2.5. We say that an $h$-homomorphism is an $h$-epimorphism if it has a right inverse that is also an $h$-homomorphism. We say that an $h$-homomorphism is an $h$ monomorphism if it has a left inverse that is also an $h$-homomorphism.

In Section 7.2, we will characterize both $h$-epimorphisms and $h$-monomorphisms by their property of factorizing either the domain or the codomain.

Definition 2.6 (Differentiability). Let $\mathbb{G}$ and $\mathbb{M}$ be graded groups with homogeneous distances $d$ and $\rho$, respectively. Let $\Omega$ be an open subset of $\mathbb{G}$ and consider $f: \Omega \longrightarrow \mathbb{M}$. We say that $f$ is differentiable at $x \in \Omega$ if there exists an $h$-homomorphism $L: \mathbb{G} \longrightarrow \mathbb{M}$ such that

$$
\frac{\rho\left(f(x)^{-1} f(x h), L(h)\right)}{d(h)} \longrightarrow 0 \quad \text { as } h \rightarrow e .
$$

The $h$-homomorphism $L$ satisfying this limit is unique and it is called differential of $f$ at $x$. We denote $L$ by $D f(x)$.

Definition 2.7 (Horizontal differentiability). Let $\Omega \subset \mathbb{G}$ be an open set and let $M$ be a smooth manifold. We consider a mapping $f: \Omega \longrightarrow M$ and $x \in \Omega$. We say that $f$ is horizontally differentiable at $x$, in short $h$-differentiable at $x$, if there exists a neighborhood $U$ of the origin in the first layer $V_{1}$ such that the restriction

$$
U \ni X \longrightarrow f(x \exp X) \in M
$$

is differentiable at the origin. We say that this differential $L: H_{x} \mathbb{G} \longrightarrow T_{f(x)} M$ is the $h$-differential of $f$ at $x$ and denote it by $d_{H} f(x)$. We say that $f$ is continuously $h$-differentiable in the case when $x \longrightarrow d_{H} f(x)$ is a continuous mapping. We also use the convenient notation $d_{H} f(x)(X)=X f(x)$.

Proposition 2.8. The differentiability of vector-valued mappings implies their h-differentiability.

Proof. Let $\|\cdot\|$ be any norm on the Lie algebra $\mathcal{G}$ of $\mathbb{G}$, let $\Omega \subset \mathbb{G}$ be an open subset and let $f: \Omega \longrightarrow V$, where $V$ is a linear space. We assume that $f$ is differentiable at $x$ and restrict its differentiability property to horizontal directions. Thus, we observe that $d(\exp X) /\|X\|$ is bounded away from zero and from above with constants independent of $X$ as it varies in $V_{1}$. This immediately leads us to the $h$-differentiability of $f$ at $x$, concluding the proof.

REMARK. From the proof of Proposition 2.8 and following the corresponding notation, it is not difficult to see that whenever $f: \Omega \longrightarrow V$ is differentiable at $x$, then we also have the equalities

$$
d_{H} f(x)(X)=X f(x)=D f(x)(\exp X) \quad \text { for every } X \in V_{1} .
$$

Clearly, $h$-differentiability is in general a weaker notion than that of differentiability. On the other hand, the little regularity of $h$-differentiable mappings suffices to introduce contact mappings. 
Definition 2.9 (Contact mapping). We say that $f: \Omega \longrightarrow \mathbb{M}$ is a contact mapping if it is $h$-differentiable and $d_{H} f(x)\left(H_{x} \mathbb{G}\right) \subset H_{f(x)} \mathbb{M}$ for every $x \in \Omega$.

Remark. Clearly, $\mathbb{R}^{k}$-valued continuously $h$-differentiable mappings are automatically contact. These mappings have been already considered in [5].

Proposition 2.10 (Chain rule). Let $f: \Omega \longrightarrow \mathbb{U}$ be differentiable at $x \in \Omega$ and let $g: \Upsilon \longrightarrow \mathbb{M}$ be differentiable at $f(x) \in \Upsilon$, where $\Omega \subset \mathbb{G}$ and $\Upsilon \subset \mathbb{U}$ are open subsets and $f(\Omega) \subset \Upsilon$. Then $g \circ f: \Omega \longrightarrow \mathbb{M}$ is differentiable at $x$ and $D(g \circ f)(x)=$ $D g(f(x)) \circ D f(x)$.

The proof of this result is elementary, see [16, Proposition 3.2.5].

\section{Technical lemmas}

Throughout this section, we denote by $\mathbb{G}$ a graded group, equipped with a homogeneous distance $d$. Its Lie algebra is denoted by $\mathcal{G}$ and it has $\iota$ layers $V_{i}$, with $i=1, \ldots, \iota$. On $\mathcal{G}$, seen as a finite dimensional real vector space, we fix a norm $\|\cdot\|$. Bilinearity of brackets yields a constant $\beta>0$, such that for every $X, Y \in \mathcal{G}$

$$
\|[X, Y]\| \leq \beta\|X\|\|Y\| .
$$

Lemma 3.1. Let $v>0$ and $n=2, \ldots$, . Then there exists a constant $\alpha_{n}(v)$ only depending on $n$ and $v$ such that

$$
\left\|c_{n}(X, Y)\right\| \leq \alpha_{n}(v)\|[X, Y]\|
$$

whenever $\|X\|,\|Y\| \leq v$.

Proof. Our statement is trivial for $n=2$, as $c_{2}(X, Y)=[X, Y] / 2$. Assume that it is true for every $j=2, \ldots, n$, with $n \geq 2$. It suffices to observe that $\left[c_{k_{2 p}}(X, Y), X+Y\right] \neq 0$ in (2.3) implies $k_{2 p}>1$, then the inductive hypothesis yields

$$
\left\|c_{k_{2 p}}(X, Y)\right\| \leq \alpha_{k_{2 p}}(v)\|[X, Y]\| .
$$

Using this estimate in (2.3) and observing that $\left\|c_{k_{i}}(X, Y)\right\| \leq 2 v$ whenever $k_{i}=1$, the thesis follows.

Lemma 3.2. Let $c_{n}(X, Y)$ be as in (2.2). Then for each $n=2, \ldots, \iota$ there exists a set of real numbers $\left\{e_{n, \alpha} \mid \alpha \in\{1,2\}^{n-1}\right\}$ only depending on $\mathcal{G}$ such that for every $A_{1}, A_{2} \in \mathcal{G}$,

$$
c_{n}\left(A_{1}, A_{2}\right)=\sum_{\alpha \in\{1,2\}^{n-1}} e_{n, \alpha} L_{n}\left(A_{\alpha}, A_{1}+A_{2}\right),
$$

where $A_{\alpha}=\left(A_{\alpha_{1}}, \ldots, A_{\alpha_{n-1}}\right), L_{1}=I d_{\mathcal{G}}$ and for $n \geq 2$ the $n$-linear mapping $L_{n}: \mathcal{G}^{n} \longrightarrow$ $\mathcal{G}$ is defined by

$$
L_{n}\left(X_{1}, X_{2}, \ldots, X_{n}\right)=\left[X_{1},\left[X_{2}, \ldots,\left[X_{n-1}, X_{n}\right]\right] \ldots\right]
$$


Proof. Let $L_{1}: \mathcal{G} \longrightarrow \mathcal{G}$ be the identity mapping and let $k_{1}, \ldots, k_{p}$ be positive integers with $n=k_{1}+k_{2}+\cdots+k_{p}$, where $p \in \mathbb{N}$. Iterating the Jacobi identity of the Lie product, it is not difficult to check that

$$
\begin{aligned}
& {\left[L_{k_{1}}\left(X_{1}^{1}, \ldots, X_{k_{1}}^{1}\right),\left[L_{k_{2}}\left(X_{1}^{2}, \ldots, X_{k_{2}}^{2}\right), \ldots,\right.\right.} \\
& \left.\left.\quad\left[L_{k_{p-1}}\left(X_{1}^{p-1}, \ldots, X_{k_{p-1}}^{p-1}\right), L_{k_{p}}\left(X_{1}^{p}, \ldots, X_{k_{p}}^{p}\right)\right]\right] \ldots\right] \\
& \quad=\sum_{\sigma_{1} \in S_{k_{1}}, \ldots, \sigma_{p-1} \in S_{k_{p-1}}} s_{\sigma_{1}, \ldots, \sigma_{p-1}} L_{n}\left(X_{\sigma_{1}}^{1}, X_{\sigma_{2}}^{2}, \ldots, X_{\sigma_{p-1}}^{p-1}, X^{p}\right),
\end{aligned}
$$

where $S_{j}$ is the set of permutations on $j$ elements, $s_{\sigma_{1}, \ldots, \sigma_{p-1}} \in\{-1,0,1\}$,

$$
X^{p}=\left(X_{1}^{p}, \ldots, X_{k_{p}}^{p}\right) \quad \text { and } \quad X_{\sigma_{j}}^{j}=\left(X_{\sigma_{j}(1)}^{j}, X_{\sigma_{j}(2)}^{j}, \ldots, X_{\sigma_{j}\left(k_{j}\right)}^{j}\right) .
$$

Our statement can be proved by induction. It is clearly true for $n=2$ taking $e_{1}=1 / 2$ and $e_{2}=0$, due to the formula $c_{2}\left(A_{1}, A_{2}\right)=\left[A_{1}, A_{2}\right] / 2$. Let us assume that (3.3) holds for all $c_{j}\left(A_{1}, A_{2}\right)$ with $j \leq n$. By the recurrence equation (2.3),

$$
\begin{aligned}
& (n+1) c_{n+1}\left(A_{1}, A_{2}\right)=\frac{1}{2} \sum_{\alpha \in\{1,2\}^{n-1}} e_{n, \alpha}\left[A_{1}-A_{2}, L_{n}\left(A_{\alpha}, A_{1}+A_{2}\right)\right] \\
& +\sum_{\substack{p \geq 1 \\
2 p \leq n}} K_{2 p} \sum_{\substack{k_{1}, \ldots, k_{2 p}>0 \\
k_{1}+\cdots+k_{2 p}=n}} \sum_{\substack{\alpha_{i} \in\{1,2\}^{k_{i}-1} \\
i=1, \ldots, 2 p}} e_{k_{1}, \alpha_{1}} e_{k_{2}, \alpha_{2}} \cdots e_{k_{2 p}, \alpha_{2 p}} \\
& {\left[L_{k_{1}}\left(A_{\alpha_{1}}, A_{1}+A_{2}\right),\left[\ldots,\left[L_{k_{2 p}}\left(A_{\alpha_{2 p}}, A_{1}+A_{2}\right), A_{1}+A_{2}\right]\right] \ldots\right] \text {. }}
\end{aligned}
$$

Then applying (3.5) we have proved that $c_{n+1}\left(A_{1}, A_{2}\right)$ can be represented as a linear combination of terms $L_{n+1}\left(A_{\alpha}, A_{1}+A_{2}\right)$, where $\alpha \in\{1,2\}^{n}$. This concludes our proof.

Definition 3.3. Let $X, Y \in \mathcal{G}$. The $k$ th bracket is defined by

$$
[X, Y]_{k}=\underbrace{[X,[X, \ldots,[X}_{k \text { times }}, Y]] \ldots] \text { and }[X, Y]_{0}=Y \text {. }
$$

Lemma 3.4. If $c_{n}(X, Y)$ is as defined in (2.2), $2 \leq n \leq \iota$, then we have

$$
c_{n}(X, Y)=\frac{(-1)^{n-1}}{n !}\left[\frac{Y-X}{2}, X+Y\right]_{n-1}+R_{n}(X, Y),
$$

and for every $v>0$ there exists a positive nondecreasing function $C(n, \cdot)$ such that for all $\|X\|,\|Y\| \leq v$,

$$
\left\|R_{n}(X, Y)\right\| \leq C(n, v)\|X+Y\|^{3} .
$$

Proof. Taking into account (2.3), we define $E_{n}(X, Y)$ by setting

$$
c_{n}(X, Y)=-\frac{1}{n}\left[\frac{Y-X}{2}, c_{n-1}(X, Y)\right]+E_{n}(X, Y) .
$$


We have $E_{2}(X, Y)=R_{2}(X, Y)=0$ for all $X, Y \in \mathcal{G}$, and hence for $n=2$ our claim trivially holds. Let us consider $n \geq 3$. By definition of $E_{n}$, applying both (2.3) and (3.3), for every $A_{1}, A_{2} \in \mathcal{G}$,

$$
\begin{aligned}
& E_{n}\left(A_{1}, A_{2}\right)=\sum_{\substack{p \geq 1 \\
2 p \leq n-1}} K_{2 p} \sum_{\substack{k_{1}, \ldots, k_{2 p}>0 \\
k_{1}+\cdots+k_{2 p}=n-1}} \sum_{\substack{\left.\alpha_{i} \in\{1,2\}\right\}_{i}-1 \\
i=1, \ldots, 2 p}} e_{k_{1}, \alpha_{1}} e_{k_{2}, \alpha_{2}} \cdots e_{k_{2 p}, \alpha_{2 p}} \\
& {\left[L_{k_{1}}\left(A_{\alpha_{1}}, A_{1}+A_{2}\right),\left[\ldots,\left[L_{k_{2 p}}\left(A_{\alpha_{2 p}}, A_{1}+A_{2}\right), A_{1}+A_{2}\right]\right] \ldots\right] .}
\end{aligned}
$$

As a consequence, there exist constants $\widetilde{C}_{p}>0$ such that

$$
\left\|E_{n}(X, Y)\right\| \leq \sum_{1 \leq p \leq(n-1) / 2} \widetilde{C}_{p} v^{n-2 p-1}\|X+Y\|^{2 p+1} .
$$

This immediately implies that

$$
\left\|E_{n}(X, Y)\right\| \leq \sum_{1 \leq p \leq(n-1) / 2} 4^{p} \widetilde{C}_{p} v^{n-3}\|X+Y\|^{3} .
$$

Iterating (3.8),

$$
\begin{aligned}
c_{n}(X, Y)= & \frac{(-1)^{2}}{n(n-1)}\left[\frac{Y-X}{2}, c_{n-2}(X, Y)\right]_{2}+E_{n}(X, Y) \\
& \quad-\frac{1}{n}\left[\frac{Y-X}{2}, E_{n-1}(X, Y)\right] \\
= & \frac{(-1)^{n-1}}{n !}\left[\frac{Y-X}{2}, c_{1}(X, Y)\right]_{n-1}+E_{n}(X, Y) \\
& \quad+\sum_{k=1}^{n-2} \frac{(-1)^{k}}{n(n-1) \cdots(n-k+1)}\left[\frac{Y-X}{2}, E_{n-k}(X, Y)\right]_{k} .
\end{aligned}
$$

Therefore, (3.9) concludes our proof.

REMARK. As a consequence of Lemma 3.4, we achieve the estimate

$$
\|(-\xi) \odot \eta\| \leq C(v)\|\xi-\eta\|
$$

for every $\xi, \eta \in \mathcal{G}$ satisfying $\|\xi\|,\|\eta\| \leq v$. To see this, it suffices to apply (3.6) and (3.7) to the Baker-Campbell-Hausdorff formula

$$
(-\xi) \odot \eta=-\xi+\eta+\sum_{n=2}^{\iota} c_{n}(-\xi, \eta) .
$$

A simple extension of $(+)$ in [24, page 13], see also [23], is given in the following lemma. 
Lemma 3.5. Let $\pi^{i}: \mathcal{G} \longrightarrow V_{i} \oplus \cdots \oplus V_{\iota}$ be the canonical projection onto the codomain and let $U$ be a bounded open neighborhood of the unit element e in $\mathbb{G}$. There exists a constant $K_{U}>0$, depending on $U$, such that for every $x \in U$,

$$
\left\|\pi^{i}\left(\exp ^{-1}(x)\right)\right\| \leq K_{U} d(x)^{i} .
$$

Proof. We first define $\mathcal{S}=\{v \in \mathcal{G} \mid d(\exp v)=1\}$. Let us fix $v \in \mathcal{S}$ and $s>0$ satisfying the condition $s \leq M$, with $M=\max _{x \in U} d(x)$. Then

$$
\left\|\pi^{i}\left(\delta_{s} v\right)\right\|=s^{i}\left\|\sum_{j=i}^{\iota} s^{j-i} \pi_{j}(v)\right\| \leq C s^{i},
$$

where $C=\sum_{j=i}^{\iota} M^{j-i} \max _{u \in \mathcal{S}}\left\|\pi_{j}(u)\right\|$. This concludes the proof.

Remark. From [4, Proposition 1.5], it is easy to check that there exists a constant $k(v)$ such that for all $\|\xi\| \leq v$,

$$
d(\exp \xi) \leq \kappa(v)\|\xi\|^{1 / \iota} .
$$

REMARK. As a byproduct of (3.10) and (3.11) we obtain the well-known estimate

$$
d(\exp \xi, \exp \eta) \leq C(v)\|\xi-\eta\|^{1 / \iota}
$$

for every $\xi, \eta \in \mathcal{G}$ satisfying $\|\xi\|,\|\eta\| \leq \nu$.

Remark. Notice that for every homogeneous distance $d$, there exists a constant $C_{d,\|\cdot\|}$ such that

$$
C_{d,\|\cdot\| \|}^{-1} \sum_{j=1}^{\iota}\left\|\pi_{j}(\xi)\right\|^{1 / j} \leq d(\exp \xi) \leq C_{d,\|\cdot\|} \sum_{j=1}^{\iota}\left\|\pi_{j}(\xi)\right\|^{1 / j} .
$$

In particular, for every $\xi, \eta \in \mathcal{G}$,

$$
d(\exp \xi, \exp \eta) \geq C_{d,\|\cdot\|}^{-1}\left\|\pi_{1}(\xi-\eta)\right\| .
$$

Lemma 3.6. Let $x, y \in \mathbb{G}$ and let $v>0$ be such that $d(x), d(y) \leq v$. Then there exists a constant $C(v)$ only depending on $\mathbb{G}$ and $v$ such that

$$
d\left(y^{-1} x y\right) \leq C(v)\left\|\exp ^{-1}(x)\right\|^{1 / \iota} .
$$

Proof. Let us fix $x=\exp \xi$ and $y=\exp h$. By Lemma 3.5 we find a constant $k_{v}>0$ such that $\|\xi\|,\|h\| \leq k_{v}$. The Baker-Campbell-Hausdorff formula yields

$$
(-h) \odot(\xi \odot h)=\xi+\sum_{n=2}^{\iota} c_{n}(\xi, h)+\sum_{n=2}^{\iota} c_{n}(-h, \xi \odot h) .
$$

The same formula along with (3.2) also gives

$$
\|\xi \odot h\| \leq k_{v}\left(2+\sum_{n=2}^{\iota} \alpha_{n}\left(k_{v}\right) \beta k_{v}\right)=A(v) .
$$


The bilinear estimate (3.2) also yields

$$
\left\|c_{n}(-h, \xi \odot h)\right\| \leq \alpha_{n}(A(v))\|[h, \xi \odot h]\| .
$$

Observing that $[h, \xi \odot h]=\left[h, \xi+\sum_{n=2}^{\iota} c_{n}(\xi, h)\right]$, we achieve the estimate

$$
\|[h, \xi \odot h]\| \leq \beta\|h\|\|\xi\|\left(1+\sum_{n=2}^{\iota} \alpha_{n}\left(k_{v}\right)\|h\|\right) .
$$

Joining this estimate with (3.15), we get

$$
\left\|c_{n}(-h, \xi \odot h)\right\| \leq \beta \alpha_{n}(A(v))\left(1+\sum_{n=2}^{\iota} v \alpha_{n}(v)\right)\|h\|\|\xi\| .
$$

Thus, formula (3.14) joined with estimates (3.2) and (3.16) yields $B(v)>0$ such that

$$
\|(-h) \odot \xi \odot h\| \leq\|\xi\|(1+B(v)\|h\|) .
$$

The previous inequality yields $\|(-h) \odot \xi \odot h\| \leq k_{v}\left(1+k_{v} B(v)\right)$, then (3.11) gives a constant $B_{1}(v)>0$ such that $d\left(y^{-1} x y\right) \leq B_{1}(v)\|(-h) \odot \xi \odot h\|^{1 / \iota}$. Thus, by (3.17) we get $B_{2}(v)>0$ such that $d\left(y^{-1} x y\right) \leq B_{2}(v)\|\xi\|^{1 / \iota}$. This finishes the proof.

REMARK. The previous lemma also provides another variant of its estimate. In fact, the condition $d(x) \leq v$ implies that $\left\|\exp ^{-1}(x)\right\|$ is bounded by $d(x)$ up to a factor only depending on $v$, due to Lemma 3.5. As a result, the assumption $d(x), d(y) \leq v$ gives a constant $C(v)$ such that the following estimate holds

$$
d\left(y^{-1} x y\right) \leq C(v) d(x)^{1 / \iota} .
$$

Lemma 3.7. Let $N$ be a positive integer and let $A_{j}, B_{j} \in \mathbb{G}$ with $j=1, \ldots, N$. Let $v>0$ be such that $d\left(B_{j} B_{j+1} \cdots B_{N}\right) \leq v$ and $d\left(A_{j}, B_{j}\right) \leq v$ for every $j=1, \ldots, N$. Then there exists $K_{v}>0$ such that

$$
d\left(A_{1} A_{2} \cdots A_{N}, B_{1} B_{2} \cdots B_{N}\right) \leq K_{v} \sum_{j=1}^{N} d\left(A_{j}, B_{j}\right)^{1 / \iota} .
$$

Proof. We define $\hat{B}_{j}=B_{j} B_{j+1} \cdots B_{N}$ and $\hat{A}_{j}=A_{j} A_{j+1} \cdots A_{N}$, and hence by the left invariance of $d$,

$$
d\left(\hat{A}_{1}, \hat{B}_{1}\right) \leq d\left(A_{N}, B_{N}\right)+\sum_{j=1}^{N-1} d\left(\hat{B}_{j+1} A_{j}^{-1} B_{j} \hat{B}_{j+1}\right) .
$$

Joining our hypothesis with (3.18),

$$
\begin{aligned}
& d\left(\hat{A}_{1}, \hat{B}_{1}\right) \leq d\left(A_{N}, B_{N}\right)+C(v) \sum_{j=1}^{N-1} d\left(A_{j}, B_{j}\right)^{1 / \iota} \leq d\left(A_{N}, B_{N}\right)^{1 / \iota} v^{1-1 / \iota} \\
& +C(v) \sum_{j=1}^{N-1} d\left(A_{j}, B_{j}\right)^{1 / \iota} \leq \max \left\{C(v), v^{1-1 / \iota}\right\} \sum_{j=1}^{N} d\left(A_{j}, B_{j}\right)^{1 / \iota} .
\end{aligned}
$$

This shows the validity of (3.19). 
Lemma 3.8. Let $X, Y, D_{1}, D_{2} \in \mathcal{G}$ and let

$$
v \geq \max \left\{\|X\|,\|Y\|,\left\|D_{1}\right\|,\left\|D_{2}\right\|\right\} .
$$

There exists a constant $\kappa_{n}>0$ such that for every $n=2, \ldots, \iota$,

$$
\left\|c_{n}\left(X+D_{1}, Y+D_{2}\right)-c_{n}(X, Y)\right\| \leq \kappa_{n} v^{n-1} \max \left\{\left\|D_{1}\right\|,\left\|D_{2}\right\|\right\} .
$$

Proof. We set $X=A_{1}^{1}, D_{1}=A_{1}^{2}, Y=A_{2}^{1}$ and $D_{2}=A_{2}^{2}$ and apply (3.3). Taking into account that $L_{n}$ is a multilinear mapping defined in (3.4),

$$
\begin{aligned}
c_{n}\left(A_{1}^{1}+\right. & \left.A_{1}^{2}, A_{2}^{1}+A_{2}^{2}\right) \\
= & \sum_{\alpha \in\{1,2\}^{n-1}} e_{n, \alpha} L_{n}\left(A_{\alpha_{1}}^{1}+A_{\alpha_{1}}^{2}, \ldots, A_{\alpha_{n-1}}^{1}+A_{\alpha_{n-1}}^{2}, A_{1}^{1}+A_{1}^{2}+A_{2}^{1}+A_{2}^{2}\right) \\
= & \sum_{\alpha \in\{1,2\}^{n-1}} e_{n, \alpha} \sum_{\gamma \in\{1,2\}^{n-1}} L_{n}\left(A_{\alpha_{1}}^{\gamma_{1}}, \ldots, A_{\alpha_{n-1}}^{\gamma_{n-1}}, A_{1}^{1}+A_{1}^{2}+A_{2}^{1}+A_{2}^{2}\right) \\
= & \sum_{\alpha \in\{1,2\}^{n-1}} e_{n, \alpha} \sum_{\gamma \in\{1,2\}^{n-1}} L_{n}\left(A_{\alpha_{1}}^{\gamma_{1}}, \ldots, A_{\alpha_{n-1}}^{\gamma_{n-1}}, A_{1}^{1}+A_{2}^{1}\right) \\
& +\sum_{i=1,2} \sum_{\alpha \in\{1,2\}^{n-1}} e_{n, \alpha} \sum_{\gamma \in\{1,2\}^{n-1}} L_{n}\left(A_{\alpha_{1}}^{\gamma_{1}}, \ldots, A_{\alpha_{n-1}}^{\gamma_{n-1}}, A_{i}^{2}\right) \\
= & c_{n}\left(A_{1}^{1}, A_{2}^{1}\right)+\sum_{\alpha \in\{1,2\}^{n-1}} e_{n, \alpha} \sum_{\substack{\gamma \in\{1,2\}^{n-1} \\
\gamma \neq(1,1, \ldots, 1)}} L_{n}\left(A_{\alpha_{1}}^{\gamma_{1}}, \ldots, A_{\alpha_{n-1}}^{\gamma_{n-1}}, A_{1}^{1}+A_{2}^{1}\right) \\
& +\sum_{i=1,2} \sum_{\alpha \in\{1,2\}^{n-1}} e_{n, \alpha} \sum_{\gamma \in\{1,2\}^{n-1}} L_{n}\left(A_{\alpha_{1}}^{\gamma_{1}}, \ldots, A_{\alpha_{n-1}}^{\gamma_{n-1}}, A_{i}^{2}\right),
\end{aligned}
$$

where for the equality second to last line we have again applied formula (3.3). Taking into account the multilinear estimate $\left\|L_{n}\left(X_{1}, X_{2}, \ldots, X_{n}\right)\right\| \leq \beta^{n} \prod_{j=1}^{n}\left\|X_{j}\right\|$, where $\beta$ is given in (3.1), a short calculation shows that

$$
\left\|c_{n}\left(A_{1}^{1}+A_{1}^{2}, A_{2}^{1}+A_{2}^{2}\right)-c_{n}\left(A_{1}^{1}, A_{2}^{1}\right)\right\|
$$

is less than or equal to $2^{n+1} \beta^{n} v^{n-1} \max \left\{\left\|A_{1}^{2}\right\|,\left\|A_{2}^{2}\right\|\right\} \sum_{\alpha \in\{1,2\}^{n-1}}\left|e_{n, \alpha}\right|$. This leads us to the conclusion.

\section{Differentiability of curves in graded groups}

In this section we study differentiability properties of curves $\Gamma:[a, b] \longrightarrow \mathbb{M}$, where $\mathbb{M}$ is a graded group. We denote by $\gamma$ the corresponding curve $\exp ^{-1} \circ \Gamma$ with values in the Lie algebra $\mathcal{M}$ of $\mathbb{M}$. The components of $\gamma$ taking values in the layers $W_{i}$ are denoted by $\gamma_{i}$, and hence $\gamma=\sum_{i=1}^{v} \gamma_{i}$. We have $\gamma_{i}=\pi_{i} \circ \gamma$, where $\pi_{i}: \mathcal{M} \longrightarrow W_{i}$ is the canonical projection on layers of degree $i$ and $\mathcal{M}=W_{1} \oplus \cdots \oplus W_{v}$. We denote by $\|\cdot\|$ a fixed norm on $\mathcal{M}$. The horizontal subspace of $\mathbb{M}$ at $x \in \mathbb{M}$ is the linear space

$$
H_{x} \mathbb{M}=\left\{U(x) \in T_{x} \mathbb{M} \mid U \in W_{1}\right\},
$$

where $U(x)$ denotes the value of the vector field $U$ at $x$. 
Definition 4.1. We say that a curve $\Gamma:[a, b] \longrightarrow \mathbb{M}$ is horizontal if $\gamma:[a, b] \longrightarrow \mathcal{M}$ is absolutely continuous and for a.e. differentiability point $t \in[a, b]$ the inclusion $\dot{\gamma}(t) \in H_{\gamma(t)} \mathbb{M}$ holds.

Identifying any $T_{x} \mathbb{M}$ with $\mathcal{M}$ and applying formula (2.1) for the differential of the exponential mapping,

$$
\dot{\Gamma}(t)=\dot{\gamma}(t)-\sum_{n=2}^{v} \frac{(-1)^{n}}{n !} \operatorname{ad}(\gamma(t))^{n-1}(\dot{\gamma}(t))=\dot{\gamma}(t)-\sum_{n=2}^{v} \frac{(-1)^{n}}{n !}[\gamma(t), \dot{\gamma}(t)]_{n-1} .
$$

Then $\dot{\Gamma}(t) \in W_{1}$ if and only if $\pi_{i}\left(\dot{\gamma}(t)-\sum_{n=2}^{v}\left((-1)^{n} / n !\right)[\gamma(t), \dot{\gamma}(t)]_{n-1}\right)=0$ for all $i \geq 2$. This immediately proves the following proposition.

Proposition 4.2. Let $\gamma:[a, b] \longrightarrow \mathcal{M}$ be absolutely continuous. Then $\Gamma$ is a horizontal curve if and only if for each $i=2, \ldots, v$ and a.e. $t$,

$$
\dot{\gamma}_{i}(t)=\sum_{n=2}^{v} \frac{(-1)^{n}}{n !} \pi_{i}\left([\gamma(t), \dot{\gamma}(t)]_{n-1}\right)
$$

Definition 4.3. Let $\gamma:[a, b] \longrightarrow \mathcal{M}$ be a locally summable curve and let $\lambda \neq 0$. The sup-average of $\gamma$ at $t$ is defined as

$$
\mathcal{A}_{t}^{\lambda}(\gamma)= \begin{cases}\sup _{0 \leq \tau \leq \lambda} f_{t}^{t+\tau}\|\gamma(l)\| d l & \text { if } \lambda>0, \\ \sup _{\lambda \leq \tau \leq 0} f_{t+\tau}^{t}\|\gamma(l)\| d l & \text { if } \lambda<0 .\end{cases}
$$

Notice that for some $t$ the sup-average $\mathcal{A}_{t}^{\lambda}(\gamma)$ takes values in $[0,+\infty]$, as $\gamma$ is not necessarily bounded in a neighborhood of $t$.

Lemma 4.4. For every $i=2, \ldots, v$, there exists a strictly increasing function $\Upsilon_{i}$ : $[0,+\infty) \longrightarrow[0,+\infty)$, which only depends on $\mathcal{M}$, is infinitesimal at zero and satisfies the following property. For any horizontal curve $\Gamma:[-\alpha, \alpha] \longrightarrow \mathbb{M}$ such that $\Gamma(0)=e$,

$$
\left|\int_{0}^{\lambda}\left\|\dot{\gamma}_{i}(t)\right\| d t\right| \leq \Upsilon_{i}(L) \mathcal{A}_{0}^{\lambda}\left(\dot{\gamma}_{1}-X\right)|\lambda|^{i},
$$

where $L=\max \left\{\mathcal{A}_{0}^{-\alpha}\left(\dot{\gamma}_{1}\right), \mathcal{A}_{0}^{\alpha}\left(\dot{\gamma}_{1}\right)\right\}, X \in \mathcal{M},\|X\| \leq L$ and the function $\mathcal{A}_{0}^{\lambda}(\cdot)$ is as in Definition 4.3.

Proof. First of all, we assume that $\mathcal{A}_{0}^{\lambda}\left(\dot{\gamma}_{1}\right)<+\infty$, otherwise the proof becomes trivial. Due to Proposition 4.2, the differential equations (4.1) hold. Applying (4.1) for $i=2$,

$$
\dot{\gamma}_{2}(t)=\frac{t}{2}\left[\frac{\gamma_{1}(t)}{t}, \dot{\gamma}_{1}(t)\right]
$$


hence we can write $\dot{\gamma}_{2}(t)$ as

$$
\dot{\gamma}_{2}(t)=\frac{t}{2}\left(\left[f_{0}^{t} \dot{\gamma}_{1}(\tau) d \tau, X\right]+\left[f_{0}^{t} \dot{\gamma}_{1}(\tau) d \tau, \dot{\gamma}_{1}(t)-X\right]\right) .
$$

For every $0<s<\alpha$, it follows that $\int_{0}^{s}\left\|\dot{\gamma}_{2}(t)\right\| d t$ is less than or equal to

$$
\frac{s}{2} \int_{0}^{s}\left\|\left[f_{0}^{t} \dot{\gamma}_{1}(\tau) d \tau, X\right]\right\| d t+\frac{s}{2} \int_{0}^{s}\left\|\left[f_{0}^{t} \dot{\gamma}_{1}(\tau) d \tau, \dot{\gamma}_{1}(t)-X\right]\right\| d t .
$$

As a consequence,

$$
\int_{0}^{s}\left\|\dot{\gamma}_{2}(t)\right\| d t \leq \frac{\beta L s}{2}\left(\int_{0}^{s} \mathcal{A}_{0}^{t}\left(\dot{\gamma}_{1}-X\right) d t+s \mathcal{A}_{0}^{s}\left(\dot{\gamma}_{1}-X\right)\right) .
$$

This estimate shows that

$$
\int_{0}^{s}\left\|\dot{\gamma}_{2}(t)\right\| d t \leq \beta L s^{2} \mathcal{A}_{0}^{s}\left(\dot{\gamma}_{1}-X\right)
$$

From the grading property of the Lie algebra, (4.1) can be stated as follows:

$$
\dot{\gamma}_{j}(t)=\sum_{n=2}^{v} \frac{(-1)^{n}}{n !} \sum_{\substack{1 \leq l_{1}, \ldots, l_{n} \leq v \\ l_{1}+\cdots+l_{n}=j}}\left[\gamma_{l_{1}}(t),\left[\ldots,\left[\gamma_{l_{n-1}}(t), \dot{\gamma}_{l_{n}}(t)\right]\right] \ldots\right]
$$

for every $j=2, \ldots, v$. We will proceed by induction, assuming that for every $j=2, \ldots, i$ and every $v \geq i$, there exists a positive constant $\kappa_{j}(\beta)$ such that

$$
\int_{0}^{s}\left\|\dot{\gamma}_{j}(t)\right\| d t \leq \kappa_{j}(\beta) F_{j}(L) \mathcal{A}_{0}^{s}\left(\dot{\gamma}_{1}-X\right) s^{j},
$$

where $F_{j}:[0,+\infty) \longrightarrow[0,+\infty)$ is a strictly increasing function, that is infinitesimal at zero. We have already proved this statement for $i=2$. Using (4.3) for $j=i+1$ and applying the inductive hypothesis (4.4),

$$
\begin{aligned}
\int_{0}^{s}\left\|\dot{\gamma}_{i+1}(t)\right\| d t \leq \sum_{n=2}^{v} \frac{\beta^{n}}{n !} \sum_{\substack{1 \leq l_{1}, \ldots, l_{n} \leq v \\
l_{1}+\cdots+l_{n}=i+1}} \int_{0}^{s}\left\|\gamma_{l_{1}}(t)\right\| \cdots\left\|\gamma_{l_{n-1}}(t)\right\|\left\|\dot{\gamma}_{l_{n}}(t)\right\| d t \\
\quad \leq \sum_{n=2}^{v} \frac{\beta^{n}}{n !} \sum_{\substack{1 \leq l_{1}, \ldots, l_{n} \leq v \\
l_{1}+\cdots+l_{n}=i+1}} \int_{0}^{s}\left\|\dot{\gamma}_{l_{1}}(t)\right\| d t \int_{0}^{s}\left\|\dot{\gamma}_{l_{2}}(t)\right\| d t \cdots \int_{0}^{s}\left\|\dot{\gamma}_{l_{n}}(t)\right\| d t \\
\quad \leq \sum_{n=2}^{v} \frac{\beta^{n} s^{i+1}}{n !} \sum_{\substack{1 \leq l_{1}, \ldots, l_{n} \leq v \\
l_{1}+\cdots+l_{n}=i+1}} \kappa_{l_{1}} \cdots \kappa_{l_{n}} F_{l_{1}}(L) \cdots F_{l_{n}}(L) \mathcal{A}_{0}^{s}\left(\dot{\gamma}_{1}-X\right)^{n} \\
\quad \leq \sum_{n=2}^{v} \frac{\beta^{n} s^{i+1}}{n !} \sum_{\substack{1 \leq l_{1}, \ldots, l_{n} \leq v \\
l_{1}+\cdots+l_{n}=i+1}} \kappa_{l_{1}} \cdots \kappa_{l_{n}} F_{l_{1}}(L) \cdots F_{l_{n}}(L)(2 L)^{n-1} \mathcal{A}_{0}^{s}\left(\dot{\gamma}_{1}-X\right) .
\end{aligned}
$$


This proves the estimates (4.4) for every $j=2, \ldots, v$. To complete the proof, it suffices to apply these estimates to the Lipschitz curve $\tilde{\Gamma}(t)=\Gamma(-t)$ and replace $X$ by $-X$.

Corollary 4.5. There exists $\Upsilon:[0,+\infty) \longrightarrow[0,+\infty)$ that is strictly increasing, infinitesimal at zero and only depending on $\mathcal{M}$ such that the following property holds. Let $\Gamma:[a, b] \longrightarrow \mathbb{M}$ be a horizontal curve and assume that $t \in[a, b]$ is an approximate continuity point $t$ of $\dot{\gamma}_{1}$. Then

$$
\left\|\pi_{i}\left(\exp ^{-1}\left(\delta_{1 / h}\left(\Gamma(t)^{-1} \Gamma(t+h)\right)\right)\right)\right\| \leq \Upsilon\left(L_{t}\right) \mathcal{A}_{t}^{h}\left(\dot{\gamma}_{1}-\dot{\gamma}_{1}(t)\right)
$$

for every $i=2, \ldots, v$, where $L_{t}=\max \left\{\mathcal{A}_{t}^{b-t}\left(\dot{\gamma}_{1}\right), \mathcal{A}_{t}^{a-t}\left(\dot{\gamma}_{1}\right)\right\}$.

Proof. Clearly, $\gamma_{1}$ is absolutely continuous. Let $t$ be an approximate continuity point of $\dot{\gamma}_{1}$ and then also a differentiability point. It suffices to apply Lemma 4.4 to the curve $h \longrightarrow \Gamma(t)^{-1} \Gamma(t+h)$ with $X=\dot{\gamma}_{1}(t)$. In fact, we observe that

$$
\pi_{1}\left(\exp ^{-1}\left(\Gamma(t)^{-1} \Gamma(t+h)\right)\right)=\gamma_{1}(t+h)-\gamma_{1}(t)
$$

and that $\mathcal{A}_{0}^{\lambda}\left(\dot{\gamma}_{1}(t+\cdot)-\dot{\gamma}_{1}(t)\right)=\mathcal{A}_{t}^{\lambda}\left(\dot{\gamma}_{1}(\cdot)-\dot{\gamma}_{1}(t)\right)$. This implies that

$$
\left\|\pi_{i}\left(\exp ^{-1}\left(\Gamma(t)^{-1} \Gamma(t+h)\right)\right)\right\| \leq \Upsilon_{i}\left(L_{t}\right)|h|^{i} \mathcal{A}_{t}^{h}\left(\dot{\gamma}_{1}-\dot{\gamma}_{1}(t)\right),
$$

where the $\Upsilon_{i}$ are given in Lemma 4.4 and $L_{t}=\max \left\{\mathcal{A}_{t}^{b-t}\left(\dot{\gamma}_{1}\right), \mathcal{A}_{t}^{a-t}\left(\dot{\gamma}_{1}\right)\right\}$. Thus, setting $\Upsilon=\max _{i=2, \ldots, v} \Upsilon_{i}$ and using the definition of dilations, our claim follows.

TheOREM 4.6. There exists a nondecreasing function $\Upsilon:[0,+\infty) \longrightarrow[0,+\infty)$ only depending on $\mathcal{M}$ with the following property. If $\Gamma:[a, b] \longrightarrow \mathbb{M}$ is a horizontal curve, then for all approximate continuity points $t$ of $\dot{\gamma}_{1}$ the estimate

$$
\left\|\delta_{1 / h}\left(-h \dot{\gamma}_{1}(t) \odot(-\gamma(t)) \odot \gamma(t+h)\right)\right\| \leq \Upsilon\left(L_{t}\right) \mathcal{A}_{t}^{h}\left(\dot{\gamma}_{1}-\dot{\gamma}_{1}(t)\right)
$$

holds, where $L_{t}=\max \left\{\mathcal{A}_{t}^{b-t}\left(\dot{\gamma}_{1}\right), \mathcal{A}_{t}^{a-t}\left(\dot{\gamma}_{1}\right)\right\}$. In particular, $\Gamma$ is a.e. differentiable.

Proof. Let $t$ be an approximate continuity point of $\dot{\gamma}_{1}$ and define

$$
h \longrightarrow \Gamma(t)^{-1} \Gamma(t+h)=\exp \theta(h)=\exp \left(\theta_{1}(h)+\cdots+\theta_{v}(h)\right),
$$

with $\theta_{i}(h) \in V_{i}$. Notice that, in particular, $\theta_{1}(h)=\gamma_{1}(t+h)-\gamma_{1}(t)$. In view of (4.5), there exists a constant $K>0$ depending on $\mathcal{M}$ and a strictly increasing function $\Upsilon:[0,+\infty) \longrightarrow[0,+\infty)$ infinitesimal at zero such that

$$
\left\|\frac{\theta_{i}(h)}{h^{i}}\right\| \leq \Upsilon\left(L_{t}\right) \mathcal{A}_{t}^{h}\left(\dot{\gamma}_{1}-\dot{\gamma}_{1}(t)\right),
$$

for every $i=2, \ldots, v$, where we have set $L_{t}=\max \left\{\mathcal{A}_{t}^{b-t}\left(\dot{\gamma}_{1}\right), \mathcal{A}_{t}^{a-t}\left(\dot{\gamma}_{1}\right)\right\}$. Thus, taking into account that $t$ is a differentiability point of $\gamma_{1}$ and that 0 is an approximate continuity point of $\dot{\theta}_{1}$, we obtain that $\Gamma$ is differentiable at $t$ and that

$$
D \Gamma(t)(\lambda)=\exp \left(\lambda \dot{\gamma}_{1}(t)\right)
$$


Notice also that $\left\|\theta_{1}(h) / h-\dot{\gamma}_{1}(t)\right\| \leq \mathcal{A}_{t}^{h}\left(\dot{\gamma}_{1}-\dot{\gamma}_{1}(t)\right)$, then (4.7) yields

$$
\left\|\delta_{1 / h} \theta(h)-\dot{\gamma}_{1}(t)\right\| \leq\left[1+(v-1) \Upsilon\left(L_{t}\right)\right] \mathcal{A}_{t}^{h}\left(\dot{\gamma}_{1}-\dot{\gamma}_{1}(t)\right) .
$$

Applying (3.6) and (4.8) to

$$
-\dot{\gamma}_{1}(t) \odot \delta_{1 / h} \theta(h)=\delta_{1 / h} \theta(h)-\dot{\gamma}_{1}(t)+\sum_{n=2}^{v} c_{n}\left(-\dot{\gamma}_{1}(t), \delta_{1 / h} \theta(h)\right),
$$

we get the estimate

$$
\begin{aligned}
& \left\|-\dot{\gamma}_{1}(t) \odot \delta_{1 / h} \theta(h)\right\| \leq\left[1+(v-1) \Upsilon\left(L_{t}\right)\right] \mathcal{A}_{t}^{h}\left(\dot{\gamma}_{1}-\dot{\gamma}_{1}(t)\right) \\
& \quad+\sum_{n=2}^{v} \frac{\beta^{n-1}}{n !}\left\|\frac{\delta_{1 / h} \theta(h)+\dot{\gamma}_{1}(t)}{2}\right\|^{n-1}\left[1+(v-1) \Upsilon\left(L_{t}\right)\right] \mathcal{A}_{t}^{h}\left(\dot{\gamma}_{1}-\dot{\gamma}_{1}(t)\right) \\
& \quad+\sum_{n=2}^{v}\left\|R_{n}\left(-\dot{\gamma}_{1}(t), \delta_{1 / h} \theta(h)\right)\right\|
\end{aligned}
$$

Observing that $\left\|\dot{\gamma}_{1}(t)\right\| \leq L_{t}$ and $\mathcal{A}_{t}^{h}\left(\dot{\gamma}_{1}-\dot{\gamma}_{1}(t)\right) \leq 2 L_{t}$, inequality (4.8) implies that

$$
\left\|\delta_{1 / h} \theta(h)\right\| \leq 3 L_{t}\left[1+(v-1) \Upsilon\left(L_{t}\right)\right]=\Upsilon_{1}\left(L_{t}\right) .
$$

Then we apply (3.7) to the third line of (4.9), obtaining

$$
\begin{aligned}
\|-\dot{\gamma}_{1}(t) & \odot \delta_{1 / h} \theta(h) \| \leq\left(1+\sum_{n=2}^{v} \frac{\beta^{n-1}}{n !}\left(\frac{\Upsilon_{1}\left(L_{t}\right)+L_{t}}{2}\right)^{n-1}\right) \\
{[1} & \left.+(v-1) \Upsilon\left(L_{t}\right)\right] \mathcal{A}_{t}^{h}\left(\dot{\gamma}_{1}-\dot{\gamma}_{1}(t)\right) \\
& \left.+\left(\sum_{n=2}^{v} C\left(n, \Upsilon_{1}\left(L_{t}\right)\right)\right)\right)\left[1+(v-1) \Upsilon\left(L_{t}\right)\right]^{3} 4 L_{t}^{2} \mathcal{A}_{t}^{h}\left(\dot{\gamma}_{1}-\dot{\gamma}_{1}(t)\right)
\end{aligned}
$$

This concludes the proof.

Corollary 4.7. Let $\Omega$ be an open set of $\mathbb{G}$ and let $f: \Omega \longrightarrow \mathbb{M}$ be a Lipschitz mapping. Then $f$ is a.e. differentiable.

Proof. By Theorem 4.6, rectifiable curves in graded groups are a.e. differentiable. This extends [24, Proposition 4.1] to graded group-valued curves. Then the same arguments of [24, Proposition 3.2 and Corollaire 3.3] lead us to our claim.

4.1. Characterization of differentiable mappings. Here we denote by $\mathbb{G}$ and $\mathbb{M}$ two arbitrary graded groups, where $\mathbb{G}$ is stratified and $\Omega \subset \mathbb{G}$ is an open set. To obtain estimates on the difference quotient of differentiable mappings, we will use the following family of piecewise horizontal lines. 
Definition 4.8. Let $N$ be a positive integer, $X_{1}, \ldots, X_{m}$ be a basis of the first layer $V_{1}$, $i_{1}, \ldots, i_{N} \in\{1, \ldots, m\}$ be fixed integers, and for every $a=\left(a_{1}, \ldots, a_{N}\right) \in \mathbb{R}^{N}$ define

$$
P^{s}(a)= \begin{cases}e & \text { if } s=0, \\ \delta_{a_{1}} h_{i_{1}} \delta_{a_{2}} h_{i_{2}} \cdots \delta_{a_{s}} h_{i_{s}} & \text { if } s=1, \ldots, N,\end{cases}
$$

where $h_{1}=\exp X_{1}, \ldots, h_{m}=\exp X_{m}$ and we have assumed that $d\left(h_{i}\right)=1$.

Now, [4, Lemma 1.40] yields the following lemma.

Lemma 4.9. For every stratified group $\mathbb{G}$ there exists an integer $N$ and a family of integers $\left\{i_{1}, \ldots, i_{N}\right\} \subset\{1, \ldots, m\}$ depending on $\mathbb{G}$, such that the mapping $P^{N}$ of Definition 4.8 sends a neighborhood of the origin in $\mathbb{R}^{N}$ onto a neighborhood of the identity $e \in \mathbb{G}$.

Definition 4.10. Under the conditions of Lemma 4.9, we define the number

$$
c(\mathbb{G}, d)=\max _{\substack{s=1, \ldots, N \\ a=\left(a_{s}\right) \in\left(P^{N}\right)^{-1}\left(D_{1}\right)}}\left|a_{s}\right|,
$$

that only depends on the algebraic structure of $\mathbb{G}$ and on the homogeneous distance $d$ used to define both $P^{N}$ and the closed unit ball $D_{1}$.

Definition 4.11. Let $f: K \longrightarrow Y$ be a vector-valued continuous function on a compact metric space $(K, \rho)$. Then we define the modulus of continuity of $f$ on $K$ as

$$
\omega_{K, f}(t)=\max _{\substack{x, y \in K \\ \rho(x, y) \leq t}}\|f(x)-f(y)\| .
$$

Theorem 4.12. Let $f: \Omega \longrightarrow \mathbb{M}$ be differentiable at $x \in \Omega$, where $f=\exp \circ F$ and $F=F_{1}+\cdots+F_{v}$ with $F_{j}: \Omega \longrightarrow W_{j}$. Then every $F_{j}$ is differentiable at $x$ and we have the formulas

$$
\begin{gathered}
\pi_{1} \circ \exp ^{-1} \circ D f(x)=D F_{1}(x), \\
D F_{i}(x)(h)=\sum_{n=2}^{v} \frac{(-1)^{n}}{n !} \pi_{i}\left([F(x), D F(x)(h)]_{n-1}\right),
\end{gathered}
$$

for every $i=2, \ldots, v$ and every $h \in \mathbb{G}$.

Proof. We will use the notation $|h|=d(h)$. By definition of differentiability, $\delta_{1 /|h|}\left(f(x)^{-1} f(x h)\right)$ uniformly converges to $D f(x)\left(\delta_{1 /|h|} h\right)$ as $h \rightarrow e$, with respect to the parameter $\delta_{1 /|h|} h$ varying in a compact set. Then the difference quotients

$$
\pi_{i} \circ \exp ^{-1}\left[\delta_{1 /|h|}\left(f(x)^{-1} f(x h)\right)\right]
$$

uniformly converge for every $i=1, \ldots, v$. In other words, the difference quotient $\left(F_{1}(x h)-F_{1}(x)\right) /|h|$ along with

$$
\frac{F_{i}(x h)-F_{i}(x)+\sum_{n=2}^{v} \pi_{i}\left(c_{n}(-F(x), F(x h))\right)}{|h|^{i}}
$$


converge as $h \rightarrow 0$, whenever $i=2, \ldots, v$. In particular, $F_{1}$ is differentiable at $x$ and $\pi_{1} \circ \exp ^{-1}(D f(x)(h))=D F_{1}(x)(h)$, which implies (4.12). Writing (4.14) for $i=2$,

$$
\frac{F_{2}(x h)-F_{2}(x)-\frac{1}{2}\left[F_{1}(x), F_{1}(x h)\right]}{|h|^{2}} .
$$

The convergence of the previous quotient and differentiability of $F_{1}$ at $x$ imply that $F_{2}$ is also differentiable at $x$ and $D F_{2}(x)(h)=\left[F_{1}(x), D F_{1}(x)(h)\right] / 2$. By induction, we assume that for $j \geq 2$, the vector-valued mapping $F_{i}$ is differentiable at $x$ and that

$$
D F_{i}(x)(h)=\sum_{n=2}^{v} \frac{(-1)^{n}}{n !} \pi_{i}\left(\left[\bar{F}_{i-1}(x), D \bar{F}_{i-1}(x)(h)\right]_{n-1}\right)
$$

holds for every $i=1, \ldots, j$, where we have set $\bar{F}_{i}=F_{1}+F_{2}+\cdots+F_{i}$. By (4.14),

$$
\frac{F_{i+1}(x h)-F_{i+1}(x)+\sum_{n=2}^{v} \pi_{i+1}\left(c_{n}(-F(x), F(x h))\right)}{|h|} \longrightarrow 0 \quad \text { as } h \rightarrow 0 .
$$

Now we notice that

$$
\pi_{i+1}\left(c_{n}(-F(t), F(t+h))\right)=\pi_{i+1}\left(c_{n}\left(-\bar{F}_{i}(t), \bar{F}_{i}(t+h)\right)\right) .
$$

Furthermore, the inductive hypothesis and Lemma 3.4 ensure the existence of

$$
\lim _{|h| \rightarrow 0} \frac{c_{n}\left(-\bar{F}_{i}(x), \bar{F}_{i}(x+h)\right)}{|h|}=\frac{(-1)^{n-1}}{n !}\left[\bar{F}_{i}(x), D \bar{F}_{i}(x)(h)\right]_{n-1} .
$$

Thus, joining (4.15), (4.16) and (4.17), it follows that $D F_{i+1}(x)$ exists and

$$
D F_{i+1}(x)(h)=\sum_{n=2}^{i+1} \frac{(-1)^{n}}{n !} \pi_{i+1}\left(\left[\bar{F}_{i}(x), D \bar{F}_{i}(x)\right]_{n-1}\right) .
$$

This shows that $F: \Omega \longrightarrow \mathcal{M}$ is differentiable at $t$, and hence in the previous formula we can replace $\bar{F}_{i}$ by $F$, achieving (4.13). This ends the proof.

REMARK. Under the assumptions of the previous theorem, it is worth mentioning that the differentiability of $f$ at $x$ also implies differentiability of $F_{j}$ along the directions of $V_{i}$ with $1 \leq i \leq j$, as has been shown in [2, Lemma 3.4]. Due to Theorem 1.1, the formulas (4.13) suffice to prove differentiability, and hence derivatives along $V_{1}$ and the contact property imply the existence of higher layer derivatives.

Corollary 4.13. Let $\Gamma:[a, b] \longrightarrow \mathbb{M}$ be differentiable at $t \in[a, b]$. Then $\gamma$ is differentiable at $t$ and satisfies (4.1).

This corollary is an immediate consequence of Theorem 4.12. Next, we present the proof of Theorem 1.1. 
Proof. If $f: \Omega \longrightarrow \mathbb{M}$ is continuously differentiable, then Theorem 4.12 implies the differentiability of all the $F_{j}$ and the validity of both formulas (1.1) and (1.2). These equations give the continuity of the differential of every $F_{j}$. Then the first condition implies the second one.

Now, we assume that the second condition holds. By Proposition 2.8, differentiability of vector-valued mappings implies their $h$-differentiability, and hence all the $F_{j}$ are, in particular, $h$-differentiable. In addition, (2.4) yields the continuity of the horizontal differential. By our assumptions, formulas (1.2) hold, therefore combining these formulas with Proposition 4.2, it follows that $f$ has the contact property. Then $f$ is continuously $h$-differentiable contact, which is the third condition.

Let us assume that $f$ be continuously $h$-differentiable contact and consider

$$
\Gamma_{X}(t)=\exp \left(\sum_{j=1}^{\iota} \gamma_{X, j}(t)\right)=f\left(c_{X}(t)\right)=\exp \left(\sum_{j=1}^{\iota} F_{j}\left(c_{X}(t)\right)\right)
$$

where $c_{X}(t)=x \exp (t X)$ and $\exp ^{-1} \circ \Gamma_{X}=\gamma_{X}=\sum_{j=1}^{\iota} \gamma_{X, j} \quad$ with $t \longrightarrow \gamma_{X, j}(t) \in V_{j}$. Notice that our assumption implies that all $F_{j}$ are also continuously $h$-differentiable, then $\dot{\gamma}_{X, j}(t)=d_{H} F_{j}\left(c_{X}(t)\right)(X)=X F_{j}\left(c_{X}(t)\right)$. We know that $\Gamma_{X}$ is a horizontal curve, and we apply the key estimate (4.6) to $\Gamma_{X}$ at $t=0$, achieving

$$
\left\|\delta_{1 / h}\left(-h X F_{1}(x) \odot\left(-\gamma_{X}(0)\right) \odot \gamma_{X}(h)\right)\right\| \leq \Upsilon(L) \mathcal{A}_{0}^{h}\left(\left(X F_{1}\right) \circ c_{X}-X F_{1}(x)\right),
$$

where $\Upsilon:[0,+\infty) \longrightarrow[0,+\infty)$ is a nondecreasing function depending on $\mathbb{M}, \mathcal{A}_{0}^{h}$ is defined in (4.2) and $L=\max \left\{\mathcal{A}_{0}^{\varepsilon}\left(\dot{\gamma}_{X, 1}\right), \mathcal{A}_{0}^{-\varepsilon}\left(\dot{\gamma}_{X, 1}\right)\right\}$. Set $\sigma=\max _{\|X\| \leq 1} d(\exp X)$, and hence $c_{X}([-\varepsilon, \varepsilon]) \subset U$ for every $X \in V_{1}$ with $\|X\|=1$ and $\varepsilon<r / \sigma$, where $D_{x, r} \subset U \subset \Omega$ and $U$ is a fixed compact set. We can also assume that $U_{-r}=\left\{y \in U \mid \operatorname{dist}\left(y, U^{c}\right) \geq r\right\}$ is a neighborhood of $x$. For every $|h| \leq \varepsilon$,

$$
\mathcal{A}_{0}^{h}\left(\left(X F_{1}\right) \circ c_{X}-X F_{1}(x)\right) \leq \max _{\substack{y, z \in U \\ d(y, z) \leq \sigma|h|}}\left\|X F_{1}(z)-X F_{1}(y)\right\| .
$$

We notice that $\dot{\Gamma}_{X}(0)=X F_{1}(x)=d_{H} F_{1}(x)(X)$ and recall the notation

$$
\omega_{U, d_{H} F_{1}}(\sigma|h|)=\max _{\substack{y, z \in U \\ d(y, z) \leq \sigma|h|}}\left\|d_{H} F_{1}(y)-d_{H} F_{1}(z)\right\| .
$$

Observing that $L \leq \max _{y \in U}\left\|d_{H} F_{1}(y)\right\|$, we have that

$$
\left\|\delta_{1 / h}\left(-h X F_{1}(x) \odot\left(-\gamma_{X}(0)\right) \odot \gamma_{X}(h)\right)\right\|
$$

is less than or equal to $\Upsilon\left(\max _{y \in U}\left\|d_{H} F_{1}(y)\right\|\right) \omega_{U, d_{H} F_{1}}(\sigma|h|)$. This number is independent of $x$ and tends to zero as $h \rightarrow 0$, so taking the exponential of the elements on the lefthand side, we get the uniform convergence of

$$
\delta_{1 / h}\left(\exp \left(-h X F_{1}(y)\right)\left(f(y)^{-1} f(y \exp (h X))\right)\right.
$$


to the unit element as $h \rightarrow 0$, where $y$ varies in the smaller neighborhood $U_{-r}$ of $x$. Thus, arguing as in [24, Corollaire 3.3], we achieve the differentiability of $f$ at $x$. This shows the everywhere differentiability of $f$. In particular, the convergence of (4.18) to the unit element as $h \rightarrow 0$ gives

$$
\exp \circ D F_{1}(x)(\exp X)=\exp \circ d_{H} F_{1}(x)(X)=\exp X F_{1}(x)=D f(x)(\exp X)
$$

for every $x \in \Omega$ and $X \in V_{1}$, where the first equality follows from (2.4). Then $x \longrightarrow D f(x)_{\mid \exp V_{1}}$ is continuous. The fact that $D f(x)_{\mid \exp V_{j}}$ polynomially depends on $D f(x)_{\mid \exp V_{1}}$ concludes the proof.

\section{Absolutely continuous curves in graded groups}

This section is devoted to the characterization of absolutely continuous curves in graded groups. Let $\mathbb{M}$ and $\mathcal{M}$ denote a graded group along with its Lie algebra, equipped with a homogeneous distance $\rho$ and a norm $\|\cdot\|$, respectively.

Definition 5.1. Let $\gamma:[0, s] \longrightarrow \mathcal{M}$ be an absolutely continuous curve in $\mathcal{M}$ and let $\Sigma=\left(t_{0}, t_{1}, \ldots, t_{N}\right)$ be a partition of $[0, s]$, where $t_{0}=0, t_{i}<t_{i+1}$ and $t_{N}=s$ for some $N \in \mathbb{N}$. The associated 'sum' with respect to the group operation in $\mathcal{M}$ is defined by

$$
\sigma_{\Sigma}(\gamma)(s)=\sum_{k=0}^{N-1} \gamma\left(t_{k}\right)^{-1} \odot \gamma\left(t_{k+1}\right)
$$

We also set $\|\Sigma\|=\max _{1 \leq i \leq N}\left(t_{i}-t_{i-1}\right)$.

Lemma 5.2. Let $\gamma:[0, s] \longrightarrow \mathcal{M}$ be absolutely continuous. Then it follows that

$$
\lim _{\|\Sigma\| \rightarrow 0^{+}} \sigma_{\Sigma}(s)=\gamma(s)-\gamma(0)+\sum_{n=2}^{\iota} \frac{(-1)^{n-1}}{n !} \int_{0}^{s}[\gamma(l), \dot{\gamma}(l)]_{n-1} d l .
$$

Proof. Applying (2.2) and (5.1),

$$
\sigma_{\Sigma}(s)=\sum_{k=0}^{N-1} \sum_{n=1}^{\iota} c_{n}\left(-\gamma\left(t_{k}\right), \gamma\left(t_{k+1}\right)\right)
$$

(3.6) yields

$$
\begin{aligned}
\sigma_{\Sigma}(s)= & \gamma(s)-\gamma(0)+\sum_{k=0}^{N-1} \sum_{n=2}^{\iota} \frac{(-1)^{n-1}}{n !}\left[\mu_{k}, \gamma\left(t_{k+1}\right)-\gamma\left(t_{k}\right)\right]_{n-1} \\
& +\sum_{k=0}^{N-1} \sum_{n=2}^{\iota} R_{n}\left(-\gamma\left(t_{k}\right), \gamma\left(t_{k+1}\right)\right),
\end{aligned}
$$

where $\mu_{k}=\left(\gamma\left(t_{k}\right)+\gamma\left(t_{k+1}\right)\right) / 2$. Thus, the absolute continuity of $\gamma$ yields

$$
\sum_{k=0}^{N-1}\left[\mu_{k}, \gamma\left(t_{k+1}\right)-\gamma\left(t_{k}\right)\right]_{n-1}=\sum_{k=0}^{N-1} \int_{t_{k}}^{t_{k+1}}\left[\mu_{k}, \dot{\gamma}(l)\right]_{n-1} d l .
$$


Notice that

$$
\begin{aligned}
\sum_{k=0}^{N-1} \int_{t_{k}}^{t_{k+1}}\left[\mu_{k}, \dot{\gamma}(l)\right]_{n-1} d l= & \int_{0}^{s}[\gamma(l), \dot{\gamma}(l)]_{n-1} d l \\
& +\sum_{p=0}^{n-2} \sum_{k=0}^{N-1} \int_{t_{k}}^{t_{k+1}}\left[\mu_{k},\left[\mu_{k}-\gamma(l),[\gamma(l), \dot{\gamma}(l)]_{p}\right]\right]_{n-p-2} d l .
\end{aligned}
$$

We have the estimate

$$
\begin{aligned}
& \left\|\sum_{p=0}^{n-2} \sum_{k=0}^{N-1} \int_{t_{k}}^{t_{k+1}}\left[\mu_{k},\left[\mu_{k}-\gamma(l),[\gamma(l), \dot{\gamma}(l)]_{p}\right]\right]_{n-p-2} d l\right\| \\
& \quad \leq(p-1) \beta^{n-2} \eta(\|\Sigma\|) \max _{t \in[0, s]}\|\gamma(t)\|^{n-2} \int_{0}^{s}\|\dot{\gamma}(l)\| d l,
\end{aligned}
$$

where we have set $\eta(\tau)=\sup _{|\beta-\alpha| \leq \tau}\left|\int_{\alpha}^{\beta}\|\dot{\gamma}(l)\| d l\right|$. Since $\gamma$ is absolutely continuous, it follows that $\eta(\tau)$ goes to zero as $\tau \rightarrow 0^{+}$. This immediately proves the validity of the limit

$$
\lim _{\|\Sigma\| \rightarrow 0^{+}} \sum_{k=0}^{N-1}\left[\frac{\gamma\left(t_{k}\right)+\gamma\left(t_{k+1}\right)}{2}, \gamma\left(t_{k+1}\right)-\gamma\left(t_{k}\right)\right]_{n-1}=\int_{0}^{s}[\gamma(l), \dot{\gamma}(l)]_{n-1} d l .
$$

Taking into account (3.7), (5.3) and (5.4), the limit (5.2) follows.

Definition 5.3. Let $\Gamma:[a, b] \longrightarrow \mathbb{M}$ be continuous. Then we define

$$
\operatorname{Var}_{a}^{t} \Gamma=\sup _{\substack{t_{0}=a<t_{1}<\cdots<t_{N}=t \\ N \in \mathbb{N}}} \sum_{k=1}^{N} \rho\left(\Gamma\left(t_{k}\right), \Gamma\left(t_{k-1}\right)\right) .
$$

Theorem 5.4. Let $\Gamma:[a, b] \longrightarrow \mathbb{M}$ be a curve and define $\gamma=\exp ^{-1} \circ \Gamma=\sum_{i=1}^{v} \gamma_{i}$, where $\gamma_{i}$ takes values in $V_{i}$. Then the following statements are equivalent:

(1) $\Gamma$ is absolutely continuous;

(2) $\gamma$ is absolutely continuous and the differential equation

$$
\dot{\gamma}_{i}(t)=\sum_{n=2}^{v} \frac{(-1)^{n}}{n !} \pi_{i}\left([\gamma(t), \dot{\gamma}(t)]_{n-1}\right)
$$

is satisfied a.e. for every $i \geq 2$.

If one of the previous conditions holds, then there exists a constant $C>0$ only depending on $\rho$ and $\|\cdot\|$, such that for any $\tau_{1}<\tau_{2}$,

$$
\rho\left(\Gamma\left(\tau_{1}\right), \Gamma\left(\tau_{2}\right)\right) \leq C \int_{\tau_{1}}^{\tau_{2}}\left\|\dot{\gamma}_{1}(t)\right\| d t
$$


Proof. We first assume that $\Gamma$ is absolutely continuous with respect to a homogeneous distance $\rho$ fixed in $\mathbb{M}$. Observing that the image of $\Gamma$ is bounded and applying Lemma 3.5 with $i=1$, we immediately conclude that $\gamma$ is also absolutely continuous with respect to the norm $\|\cdot\|$ fixed in $\mathcal{M}$; then $\gamma$ is a.e. differentiable. Let $a<\tau<b$ be both an approximate continuity point of $\dot{\gamma}$ and a differentiability point of the total variation $[a, b] \ni l \longrightarrow \operatorname{Var}_{a}^{l} \Gamma$. We fix a suitably small $\varepsilon>0$. We also fix a bounded open neighborhood $U$ of $e$ and choose $\delta>0$ such that $\exp \left(\gamma\left(\tau+t_{i-1}\right)^{-1} \odot \gamma\left(\tau+t_{i}\right)\right) \in$ $U$ whenever $\Sigma=\left(t_{0}, t_{1}, \ldots, t_{N}\right)$ is an arbitrary partition of $[0, s]$ with $\|\Sigma\| \leq \delta$ and $0<s \leq \varepsilon$. For every $i=1, \ldots, v$, there exists a constant $k_{i}>0$ such that

$$
\begin{aligned}
& \left\|\pi_{i}\left(\sigma_{\Sigma}(\gamma(\tau+\cdot))(s)\right)\right\| \leq k_{i}\left\|\pi^{i}\left(\sigma_{\Sigma}(\gamma(\tau+\cdot))(s)\right)\right\| \\
& \quad \leq k_{i} K_{U} \sum_{j=1}^{N} \rho\left(\Gamma\left(\tau+t_{j}\right), \Gamma\left(\tau+t_{j-1}\right)\right)^{i} \leq k_{i} K_{U}\left(\operatorname{Var}_{\tau}^{\tau+s} \Gamma\right)^{i} .
\end{aligned}
$$

By Lemma 5.2, passing to the limit in (5.7) as $\|\Sigma\| \longrightarrow 0^{+}$,

$$
\begin{gathered}
\left\|\gamma_{i}(\tau+s)-\gamma_{i}(\tau)+\sum_{n=2}^{v} \frac{(-1)^{n-1}}{n !} \int_{0}^{s} \pi_{i}\left([\gamma(\tau+l), \dot{\gamma}(\tau+l)]_{n-1}\right) d l\right\| \\
\leq k_{i} K_{U}\left(\operatorname{Var}_{\tau}^{\tau+s} \Gamma\right)^{i-1}\left(\operatorname{Var}_{a}^{\tau+s} \Gamma-\operatorname{Var}_{a}^{\tau} \Gamma\right) .
\end{gathered}
$$

By our assumptions, $\tau$ is a differentiability point of both $\gamma$ and $l \longrightarrow \operatorname{Var}_{a}^{l} \Gamma$, and it is an approximate continuity point of $\dot{\gamma}$. Thus, dividing the last inequality by $s$ and taking the limit as $s \rightarrow 0^{+}$for every $i \geq 2$, (5.5) follows.

Now, we assume that $\gamma$ is absolutely continuous and that (5.5) holds a.e. for every $i \geq 2$. Let us fix $a<\tau_{1}<\tau_{2}<b$ and consider

$$
h \longrightarrow \Theta(h)=\Gamma\left(\tau_{1}\right)^{-1} \Gamma\left(\tau_{1}+h\right)=\exp \theta(h)=\exp \left(\theta_{1}(h)+\theta_{2}(h)+\cdots+\theta_{v}(h)\right),
$$

where $\theta_{i}(h) \in V_{i}$. Since $h \rightarrow \Theta(h)$ is a left translate of $h \rightarrow \Gamma\left(\tau_{1}+h\right)$, where the latter is horizontal a.e., it follows that $\Theta$ is horizontal a.e., and we can conclude that

$$
\dot{\theta}_{i}(h)=\sum_{n=2}^{v} \frac{(-1)^{n}}{n !} \pi_{i}\left([\theta(h), \dot{\theta}(h)]_{n-1}\right)
$$

and that $\theta$ is absolutely continuous. Now, we wish to prove by induction that there exists a constant $C_{i}>0$, only depending on the norm constant $\beta$, such that

$$
\int_{0}^{h_{0}}\left\|\dot{\theta}_{i}(t)\right\| d t \leq C_{i}\left(\int_{0}^{h_{0}}\left\|\dot{\theta}_{1}\right\|\right)^{i}
$$

for every $i \geq 1$, with $h_{0}=\tau_{2}-\tau_{1}$. To prove (5.9), we first write (5.8) as

$$
\dot{\theta}_{i}(h)=\sum_{n=2}^{v} \frac{(-1)^{n}}{n !} \sum_{\substack{1 \leq l_{1}, \ldots, l_{n} \leq v \\ l_{1}+\cdots+l_{n}=i}}\left[\theta_{l_{1}}(h),\left[\theta_{l_{2}}(h), \ldots,\left[\theta_{l_{n-1}}(h), \dot{\theta}_{l_{n}}(h)\right]\right] \ldots\right],
$$


since $\mathbb{M}$ is a graded group. For $i=1$, inequality (5.9) is trivial, choosing $C_{1} \geq 1$. Let us assume that (5.9) holds for every $i \leq j$. Due to (5.10) for $i=j+1$ and our induction hypothesis,

$$
\begin{aligned}
\int_{0}^{h_{0}} & \left\|\dot{\theta}_{j+1}(t)\right\| d t \leq \sum_{n=2}^{v} \frac{\beta^{n-1}}{n !} \sum_{\substack{1 \leq l_{1}, \ldots, l_{n} \leq v \\
l_{1}+\cdots+l_{n}=j+1}} \int_{0}^{h_{0}}\left\|\theta_{l_{1}}(t)\right\| \ldots\left\|\theta_{l_{n-1}}(t)\right\|\left\|\dot{\theta}_{l_{n}}(t)\right\| d t \\
& \leq \sum_{n=2}^{v} \frac{\beta^{n-1}}{n !} \sum_{\substack{1 \leq l_{1}, \ldots, l_{n} \leq v \\
l_{1}+\cdots+l_{n}=j+1}} C_{l_{1}} \cdots C_{l_{n}}\left(\int_{0}^{h_{0}}\left\|\dot{\theta}_{1}(t)\right\| d t\right)^{j+1} \\
& =\left(\int_{0}^{h_{0}}\left\|\dot{\theta}_{1}(t)\right\| d t\right)^{j+1}\left(\sum_{n=2}^{v} \frac{\beta^{n-1}}{n !} \sum_{\substack{1 \leq l_{1}, \ldots, l_{n} \leq v \\
l_{1}+\cdots+l_{n}=j+1}} C_{l_{1}} \cdots C_{l_{n}}\right) .
\end{aligned}
$$

In fact, in the previous formulas $l_{1}, \ldots, l_{n} \leq j$, and hence we can apply the induction hypothesis to $\int_{0}^{h_{0}}\left\|\dot{\theta}_{l_{j}}(t)\right\| d t$ for all $j=1, \ldots, n$. Thus, for all $i=1, \ldots, v$,

$$
\left\|\theta_{i}\left(h_{0}\right)\right\|=\left\|\pi_{i}\left(-\gamma\left(\tau_{1}\right) \odot \gamma\left(\tau_{2}\right)\right)\right\| \leq C_{i}\left(\int_{\tau_{1}}^{\tau_{2}}\left\|\dot{\gamma}_{1}\right\|\right)^{i} .
$$

These estimates establish (5.6), and hence $\Gamma$ is absolutely continuous.

Corollary 5.5. Let $\Gamma:[a, b] \longrightarrow \mathbb{M}$, let $\gamma=\exp ^{-1} \circ \Gamma$ and define $\gamma=\sum_{i=1}^{v} \gamma_{i}$, where $\gamma_{i}$ takes values in $V_{i}$. Then the following statements are equivalent:

(1) $\Gamma$ is Lipschitz;

(2) $\gamma$ is Lipschitz and the differential equation

$$
\dot{\gamma}_{i}(t)=\sum_{n=2}^{v} \frac{(-1)^{n}}{n !} \pi_{i}\left([\gamma(t), \dot{\gamma}(t)]_{n-1}\right)
$$

is satisfied a.e. for every $i \geq 2$.

If one of the previous conditions holds, then there exists a constant $C>0$ only depending on $\rho$ and $\|\cdot\|$, such that

$$
C^{-1} \operatorname{Lip}\left(\gamma_{1}\right) \leq \operatorname{Lip}(\Gamma) \leq C \operatorname{Lip}\left(\gamma_{1}\right)
$$

Remark. Notice that in Carnot-Carathéodory spaces 1-Lipschitz curves with respect to the Carnot-Carathéodory distance coincide with subunit curves, which are horizontal by definition [9]. On the other hand, the previous corollary treats the case where $\mathbb{M}$ is a graded group, that need not be a Carnot-Carathéodory space.

The metric derivative of absolutely continuous curves suffices to recover by integration their total variation. Since Theorem 4.6 and Theorem 5.4 show that absolutely continuous curves are a.e. differentiable, the following corollary follows. 
Corollary 5.6. Let $\Gamma:[a, b] \longrightarrow \mathbb{M}$ be absolutely continuous. Then

$$
\operatorname{Var}_{a}^{b} \Gamma=\int_{a}^{b} \rho\left(\exp \left(\dot{\gamma}_{1}(t)\right)\right) d t
$$

where $\operatorname{Var}_{a}^{b} \Gamma$ is computed with respect to the homogeneous distance $\rho$.

\section{Mean value estimate}

Throughout this section, $\mathbb{G}$ and $\mathcal{G}$ denote a stratified group and its Lie algebra, $\Omega \subset \mathbb{G}$ is a fixed open set, $d$ is a homogeneous distance on $\mathbb{G}$, and $\mathbb{M}$ is a graded group equipped with a homogeneous distance $\rho$. When the norm $\|\cdot\|$ is applied to a vector of either $T \mathbb{G}$ or $T \mathbb{M}$, it is understood that it represents the left invariant Finsler norm generated by the fixed norm in the Lie algebra of either $\mathbb{G}$ or $\mathbb{M}$. When we wish to stress the dependence on the point, we use the notation $\|v\|_{x}$, that is precisely $\left\|d l_{x}^{-1} v\right\|$, where $v \in T_{x} \mathbb{G}$ and $l_{x}(z)=x z$ is the left translation by $x$. The mapping $f: \Omega \longrightarrow \mathbb{M}$ will be represented in the algebra by $F=\exp ^{-1} \circ f: \Omega \longrightarrow \mathcal{M}$ and $F_{j}=\pi_{j} \circ F$, where $\pi_{j}: \mathcal{M} \longrightarrow W_{j}$ are the canonical projections onto the layers.

Lemma 6.1. Let $V$ be a finite dimensional vector space equipped with a norm $\|\cdot\|$, and let $F: \Omega \longrightarrow V$ be continuously differentiable. There exist constants $\mu_{0}>1$ and $C_{0}>0$, only depending on $(\mathbb{G}, d)$, such that

$$
\|F(x)-F(y)\| \leq C_{0} \max _{z \in D_{\xi, \mu_{0} r}}\left\|d_{H} F(z)\right\| d(x, y)
$$

for every $x, y \in D_{\xi, r}$, where $D_{\xi, \mu_{0} r} \subset \Omega$.

This lemma can be achieved by integration on a fixed family of piecewise horizontal lines, as in the proof of Theorem 1.2. Notice that the operator norm $\left\|d_{H} F(z)\right\|$ is defined as $\max \left\{\left\|d_{H} F(z)(v)\right\|: v \in H_{z} \mathbb{G},\|v\|_{z}=1\right\}$, so

$$
\left\|d_{H} F(z)(X)\right\| \leq\left\|d_{H} F(z)\right\|\|X(z)\|_{z}=\left\|d_{H} F(z)\right\|\|X\| .
$$

Combining Lemma 6.1, Theorem 4.12 and Proposition 2.10, we immediately arrive at the next proposition.

Proposition 6.2. Let $c:[a, b] \longrightarrow \Omega$ be a Lipschitz curve and let $f: \Omega \longrightarrow \mathbb{M}$ be a continuously differentiable mapping. Then there exists a constant $C>0$, only depending on $d, \rho$ and the norms on $\mathcal{G}$ and $\mathcal{M}$, such that $\Gamma=f \circ c$ is Lipschitz and

$$
\begin{gathered}
\rho(\Gamma(t), \Gamma(\tau)) \leq C \operatorname{Lip}(c) \max _{x \in c([a, b])}\left\|d_{H} F_{1}(x)\right\||t-\tau|, \\
\dot{\gamma}_{1}(t)=D F_{1}(c(t)) \circ D c(t)=D F_{1}(c(t))\left(\exp \dot{\alpha}_{1}(t)\right),
\end{gathered}
$$

where we have set $\gamma_{j}=\pi_{j} \circ \exp ^{-1} \circ \Gamma$ and $\alpha_{j}=\pi_{j} \circ \exp ^{-1} \circ c$, and hence

$$
\Gamma=\exp \left(\gamma_{1}+\cdots+\gamma_{v}\right) \text { and } c=\exp \left(\alpha_{1}+\cdots+\alpha_{\iota}\right) .
$$


COROLlary 6.3. Every continuously differentiable mapping is locally Lipschitz.

Theorem 6.4. Let $f: \Omega \longrightarrow \mathbb{M}$ be continuously differentiable, let $\Omega^{\prime}$ be an open subset compactly contained in $\Omega$ and let $c:[a, b] \longrightarrow \Omega^{\prime}$ be Lipschitz. Then there exists $C>0$, depending on both $\lambda=\operatorname{ess} \sup _{[a, b]}\|\dot{\alpha}\|$ and $L=\max _{x \in \overline{\Omega^{\prime}}}\left\|d_{H} F_{1}(x)\right\|$, such that at all approximate continuity points $t$ of $\dot{c}$,

$$
\begin{aligned}
& \rho\left(D f(c(t))(\exp \dot{c}(t) h), f(c(t))^{-1} f(c(t+h))\right) \\
& \quad \leq C \sup _{s \in I_{t, t+h}}\left|f_{t}^{s}\left\|\dot{\alpha}_{1}(l)-\dot{\alpha}_{1}(t)\right\| d l\right|^{1 / \iota}|h|+C \max _{s \in I_{t, t+h}}\left\|d_{H} F_{1}(c(s))-d_{H} F_{1}(c(t))\right\|^{1 / \iota}|h|,
\end{aligned}
$$

where $c=\exp \left(\alpha_{1}+\cdots+\alpha_{1}\right)$ and $I_{t, t+h}=[\min \{t, t+h\}, \max \{t, t+h\}] \subset[a, b]$.

Proof. By Proposition 6.2, it follows that $\Gamma=f \circ c=\exp \circ \gamma$ is Lipschitz and

$$
\dot{\gamma}_{1}(t)=D F_{1}(c(t))\left(\exp \dot{\alpha}_{1}(t)\right) .
$$

Thus, by Theorem 4.6 and taking into account (6.2), the estimate (4.6) applied to $\Gamma$ yields

$$
\begin{aligned}
\| \delta_{1 / h}[ & -h d F_{1}(c(t))\left(\dot{\alpha}_{1}(t)\right) \odot(-F(c(t)) \odot F(c(t+h))] \| \\
\leq & \Upsilon(\lambda L) \sup _{s \in I_{t, t+h}}\left|f_{t}^{s}\left\|D F_{1}(c(l))\left(\exp \left(\dot{\alpha}_{1}(l)\right)\right)-D F_{1}(c(t))\left(\exp \left(\dot{\alpha}_{1}(t)\right)\right)\right\| d l\right| \\
\leq & \Upsilon(\lambda L) L \sup _{s \in I_{t, t+h}}\left|f_{t}^{s}\left\|\dot{\alpha}_{1}(l)-\dot{\alpha}_{1}(t)\right\| d l\right| \\
& +\Upsilon(\lambda L) \lambda \max _{s \in I_{t, t+h}}\left\|d_{H} F_{1}(c(s))-d_{H} F_{1}(c(t))\right\|,
\end{aligned}
$$

where $\Upsilon$ is defined in Theorem 4.6. Now, applying (3.12) and (6.3), we obtain a constant $C>0$ such that (6.1) holds.

CoRollary 6.5. Let $f: \Omega \longrightarrow \mathbb{M}$ be a continuously differentiable mapping and let $\Omega_{1}$ and $\Omega_{2}$ be open subsets such that $\Omega_{2}$ is compactly contained in $\Omega$. Let $x, y \in \Omega_{1}$ be such that $x^{-1} y \in \exp V_{1}$ and $x \delta_{l}\left(x^{-1} y\right) \in \bar{\Omega}_{2}$, whenever $0 \leq l \leq 1$. Then there exists $a$ constant $C$, only depending on $\max _{x \in \bar{\Omega}_{2}}\left\|d_{H} F_{1}(x)\right\|$, such that

$$
\rho\left(D f(x)\left(x^{-1} y\right), f(x)^{-1} f(y)\right) \leq C \omega_{\bar{\Omega}_{2}, d_{H} F_{1}}(d(x, y))^{1 / \iota} d(x, y) .
$$

Next, we present the proof of Theorem 1.2.

Proof. Let $P^{s}$ be as in Definition 4.8, with $s=1, \ldots, N$, where $N$ is defined in Lemma 4.9. Let $x, y \in \Omega_{1}$ and let $a \in\left(P^{N}\right)^{-1}\left(D_{1}\right)$ be such that $P^{N}(a)=$ $\delta_{1 / d(x, y)}\left(x^{-1} y\right)$. We have $d\left(P^{s}(a)\right) \leq s c_{0}$, where $c_{0}=c(\mathbb{G}, d)$ is given in Definition 4.10. Then $d\left(\delta_{t} P^{s-1}(a) \delta_{l} h_{i_{s}}\right) \leq c_{0} N \operatorname{diam}\left(\Omega_{1}\right)$, when $l \in\left[0, t a_{s}\right]$ and $0 \leq t \leq \operatorname{diam}\left(\Omega_{1}\right)$. We fix $t_{0}=d(x, y) \leq \operatorname{diam}\left(\Omega_{1}\right)$, and hence $\left[0, t_{0} a_{s}\right] \ni l \longrightarrow x \delta_{t_{0}} P^{s-1}(a) \delta_{l} h_{i_{s}} \in \Omega_{2}$. 
Applying (6.4) to this curve, we get $C_{1}$, only depending on $\max _{x \in \bar{\Omega}_{2}}\left\|d_{H} F_{1}(x)\right\|$, such that

$$
\begin{aligned}
& \rho\left(f\left(x \delta_{t_{0}} P^{s-1}(a)\right)^{-1} f\left(x \delta_{t_{0}} P^{s}(a)\right), D f\left(x \delta_{t_{0}} P^{s-1}(a)\right)\left(\delta_{a_{s} t_{0}} h_{i_{s}}\right)\right) \\
& \quad \leq C_{1}\left|a_{s} t_{0}\right| \omega_{\bar{\Omega}_{2}, d_{H} F_{1}}\left(c_{0} t_{0}\right)^{1 / \iota} .
\end{aligned}
$$

Then we obtain the key uniform estimate

$$
\begin{aligned}
& \rho\left(\delta_{1 / t_{0}}\left(f\left(x \delta_{t_{0}} P^{s-1}(a)\right)^{-1} f\left(x \delta_{t_{0}} P^{s}(a)\right)\right), D f\left(x \delta_{t_{0}} P^{s-1}(a)\right)\left(\delta_{a_{s}} h_{i_{s}}\right)\right) \\
& \leq C_{1} c_{0} \omega_{\bar{\Omega}_{2}, d_{H} F_{1}}\left(c_{0} t_{0}\right)^{1 / \iota} .
\end{aligned}
$$

Let $v=\max _{x \in \bar{\Omega}_{2}}\left\|d_{H} F_{1}(x)\right\|$ and let $h_{i}=\exp X_{i}$ for $i=1, \ldots, m$. By (1.1), we have a geometric constant $c_{1}>0$ such that

$$
\rho\left(D f(x)\left(h_{i_{s}}\right)\right)=\rho\left(\exp X_{i_{s}} F_{1}(x)\right) \leq c_{1} v
$$

for all $i_{s}$. Then we have the estimate

$$
\rho\left(D f(x)\left(\delta_{a_{s}} h_{i_{s}} \cdots \delta_{a_{N}} h_{i_{N}}\right)\right) \leq c_{0} c_{1} N v .
$$

Taking into account (3.11), we have $\kappa_{0}>0$ depending on both $v$ and $c_{0}$ such that

$$
\rho\left(D f(x)\left(\delta_{a_{s}} h_{i_{s}}\right), D f\left(x \delta_{t_{0}} P^{s-1}(a)\right)\left(\delta_{a_{s}} h_{i_{s}}\right)\right) \leq \kappa_{0} c_{0}^{1 / \iota} \omega_{\bar{\Omega}_{2}, d_{H} F_{1}}\left(c_{0} N t_{0}\right)^{1 / \iota} .
$$

Joining (6.5) and (6.7), for all $s=1, \ldots, N$, we get

$$
\begin{gathered}
\rho\left(\delta_{1 / t_{0}}\left(f\left(x \delta_{t_{0}} P^{s-1}(a)\right)^{-1} f\left(x \delta_{t_{0}} P^{s}(a)\right)\right), D f(x)\left(\delta_{a_{s}} h_{i_{s}}\right)\right) \\
\leq\left(\kappa_{0} c_{0}^{1 / \iota}+C_{1} c_{0}\right) \omega_{\bar{\Omega}_{2}, d_{H} F_{1}}\left(N c_{0} t_{0}\right)^{1 / \iota} \leq C_{2},
\end{gathered}
$$

where $C_{2}$ depends on $C_{1}, c_{0}, \kappa_{0}, \iota$ and $\omega_{\Omega_{2}, d_{H} F_{1}}\left(\operatorname{diam}\left(\Omega_{2}\right)\right)$. By (6.6) and (6.8), in view of Lemma 3.7, we get a constant $\kappa_{1}$, possibly depending on both $v$ and $\omega_{\bar{\Omega}_{2}, d_{H} F_{1}}\left(\operatorname{diam}\left(\Omega_{2}\right)\right)$, such that

$$
\begin{aligned}
& \rho\left(\delta_{1 / t_{0}}\left(f(x)^{-1} f\left(x \delta_{t_{0}} P^{N}(a)\right)\right), D f(x)\left(P^{N}(a)\right)\right) \\
& \quad \leq \kappa_{1} \sum_{s=1}^{N} \rho\left(\delta_{1 / t_{0}}\left(f\left(x \delta_{t_{0}} P^{s-1}(a)\right)^{-1} f\left(x \delta_{t_{0}} P^{s}(a)\right)\right), D f(x)\left(\delta_{a_{s}} h_{i_{s}}\right)\right)^{1 / \iota} .
\end{aligned}
$$

Thus, due to (6.8), we achieve

$$
\frac{\rho\left(f(x)^{-1} f(y), D f(x)\left(x^{-1} y\right)\right)}{d(x, y)} \leq N \kappa_{1}\left(\kappa_{0} c_{0}^{1 / \iota}+C_{1} c_{0}\right)^{1 / \iota} \omega_{\bar{\Omega}_{2}, d_{H} F_{1}}\left(N c_{0} t_{0}\right)^{1 / \iota^{2}} .
$$

This estimate clearly extends to $\bar{\Omega}_{1}$ and concludes the proof. 
Next, we give the proof of Theorem 1.3.

Proof. By continuity of $x \longrightarrow D f(x)$, there exist a compact neighborhood $U^{\prime}$ of $\bar{x}$ and a number $\mu>0$ such that

$$
\min _{x \in U^{\prime}} \min _{d(u)=1} \rho(D f(x)(u))=\mu .
$$

Then the triangle inequality joined with (1.6) gives us $\beta>0$ and an open set $U$ such that $d(f(x), f(y)) \geq \beta d(x, y)$, whenever $x, y \in U$ and $\bar{x} \in U \subset U^{\prime}$. Then $f_{\mid U}$ has an inverse defined on $f(U)$ and by the domain invariance theorem, see, for instance, [14, Theorem 3.3.2], $f_{\mid U}$ is an open mapping. Then the inverse mapping $g: f(U) \longrightarrow U$ is continuous and Proposition 2.10 concludes the proof.

\section{Homogeneous subgroups}

Throughout this section, we assume that all subgroups are closed, connected and simply connected Lie subgroups, if not otherwise stated. Here $\mathbb{G}$ denotes an arbitrary graded group, that is not necessarily stratified. Its Lie algebra is denoted by $\mathcal{G}$. From [27, Section 5.2.4] we recall the notion of homogeneous subalgebra and the corresponding notion of homogeneous subgroup.

Definition 7.1 (Homogeneous subalgebra). Let $\mathfrak{p} \subset \mathcal{G}$ be a Lie subalgebra. We say that $\mathfrak{p}$ is a homogeneous subalgebra if $\delta_{r} \mathfrak{p} \subset \mathfrak{p}$ for every $r>0$.

REMARK. It is not difficult to find examples of subalgebras which are not homogeneous. It suffices to consider $\mathcal{L}=\operatorname{span}\{X+Z\}$, which is a subalgebra of the Heisenberg algebra $\mathfrak{h}^{1}$ of brackets $[X, Y]=Z$. However, $\delta_{2}(X+Z)=2 X+4 Z \notin \mathcal{L}$.

Proposition 7.2. Let a be a homogeneous subalgebra of $\mathcal{G}$, where $\mathcal{G}$ is decomposed into the direct sum $V_{1} \oplus \cdots \oplus V_{\iota}$. Then we have $\mathfrak{a}=\left(V_{1} \cap \mathfrak{a}\right) \oplus\left(V_{2} \cap \mathfrak{a}\right) \oplus \cdots \oplus$ $\left(V_{\iota} \cap \mathfrak{a}\right)$.

Proof. We have to prove that each $\xi \in \mathfrak{a}$ with the unique decomposition $\xi=\sum_{j=1}^{\iota} \xi_{j}$, with $\xi_{j} \in V_{j}$, satisfies $\xi_{j} \in \mathfrak{a}$. By hypothesis, $\delta_{r} \xi \in \mathfrak{a}$ whenever $r>0$, so the closedness of a implies that $\lim _{r \rightarrow 0^{+}} r^{-1} \delta_{r} \xi=\xi_{1} \in \mathfrak{a}$. This implies that $\xi-\xi_{1} \in \mathfrak{a}$, and so $\lim _{r \rightarrow 0^{+}} r^{-2} \delta_{r}\left(\xi-\xi_{1}\right)=\xi_{2} \in \mathfrak{a}$. Iterating this argument, our claim follows.

COROLlaRY 7.3. Every homogeneous subalgebra $\mathfrak{a} \subset \mathcal{G}$ is a graded algebra.

ReMARK. Let $\mathfrak{a}=\mathfrak{a}_{1} \oplus \cdots \oplus \mathfrak{a}_{\iota}$ be a homogeneous subalgebra. In general, some factor $\mathfrak{a}_{j}$ might be the null space. Consider, for instance, the homogeneous subalgebra $\mathfrak{a}=V_{2} \oplus V_{4} \oplus \cdots \oplus V_{2[\iota / 2]}$, where $\mathcal{G}=V_{1} \oplus V_{2} \oplus \cdots \oplus V_{\iota}$.

Definition 7.4 (Homogeneous subgroup). Let $P \subset \mathbb{G}$ be a Lie subgroup. We say that $P$ is a homogeneous subgroup if $\delta_{r} P \subset P$ for every $r>0$.

It is clear that all properties of homogeneous subalgebras are exactly translated to homogeneous subgroups. Thus, in the sequel we will equivalently work with either homogeneous subalgebras or homogeneous subgroups. 
7.1. Complementary subgroups. This subsection is devoted to the notion of complementary subgroup, which plays an important role in the algebraic side of this paper.

When $A$ and $B$ are subsets of an abstract group $G$ we will use the notation

$$
A B=\{a b \mid a \in A, b \in B\} .
$$

Definition 7.5 (Complementary subgroup). Let $P$ be a homogeneous subgroup of $\mathbb{G}$. If there exists a homogeneous subgroup $H$ of $\mathbb{G}$ satisfying the properties $P H=\mathbb{G}$ and $P \cap H=\{e\}$, then we say that $H$ is a complementary subgroup to $P$.

REMARK. Recall that for abstract subgroups $A, B$ of an abstract group $G$, the subset $A B$ is an abstract subgroup if and only if $A B=B A$, see, for instance, [11]. As a consequence, $H$ is complementary to $P$ if and only if $P$ is complementary to $H$.

Due to the previous remark, being complementary is a symmetric relation and we can say that two subgroups are complementary.

Remark. Let $H$ and $P$ be complementary subgroups of $\mathbb{G}$ and let $g \in \mathbb{G}$. Then it is immediate to check that there exist unique elements $h, h^{\prime} \in H$ and $p, p^{\prime} \in P$ such that $g=h p=p^{\prime} h^{\prime}$.

The following proposition characterizes complementary subgroups by their Lie subalgebras. Its proof is elementary and uses intrinsic dilations.

Proposition 7.6. Let $\mathfrak{p}$ and $\mathfrak{h}$ be homogeneous subalgebras of $\mathcal{G}$ and let $P$ and $H$ denote their corresponding homogeneous subgroups, respectively. Then the condition $\mathfrak{p} \oplus \mathfrak{h}=\mathcal{G}$ is equivalent to require that $P$ and $H$ are complementary subgroups. Furthermore, if one of these conditions hold, then the following mapping is a diffeomophism:

$$
\phi: \mathfrak{p} \times \mathfrak{h} \longrightarrow \mathbb{G}, \quad \phi(W, Y)=\exp W \exp Y .
$$

Remark. Joining Propositions 7.2 and 7.6, we have the following property. If $K$ and $P$ are complementary subgroups, then

$$
Q=\mathcal{H}-\operatorname{dim}(K)+\mathcal{H}-\operatorname{dim}(P),
$$

where $\mathcal{H}$-dim denotes the Hausdorff dimension with respect to a fixed homogeneous distance. One can interpret this fact as a 'proper splitting' of the ambient group even with respect to the metric point of view.

Through Proposition 7.6, it is easy to observe that not every subgroup admits a complementary subgroup.

EXAMPLE 7.7. Let us consider the second layer $n=\operatorname{span}\{Z\}$ of the Heisenberg algebra $\mathfrak{h}^{1}$, with bracket relations $[X, Y]=Z$. Then the normal subgroup $N=\exp (\mathfrak{r})$ does not possess any complementary subgroup, see also [7]. In fact, let $\mathfrak{a}$ be any 
two-dimensional homogeneous subalgebra of $\mathfrak{h}^{1}$. Then Proposition 7.2 gives the decomposition $\mathfrak{a}=\mathfrak{a}_{1} \oplus \mathfrak{a}_{2}$, where $\mathfrak{a}_{j}$ is contained in the $j$ th layer, $j=1,2$. If $\mathfrak{a}_{2}=\{0\}$, then $\mathfrak{a}=\mathfrak{a}_{1}=\mathfrak{v}$ is not a subalgebra of $\mathfrak{h}^{1}$. Thus, $\mathfrak{a}$ must contain $\mathfrak{n}$ and this conflicts with existence of a two-dimensional subalgebra complementary to $\mathrm{n}$.

REMARK. More generally, there do not exist two-dimensional subalgebras $\mathfrak{a}$ of $\mathfrak{h}^{1}$ such that $\mathfrak{a} \oplus \mathfrak{n}=\mathfrak{h}^{1}$, even if we do not require the homogeneity of $\mathfrak{a}$. In fact, let $\mathfrak{a}=\operatorname{span}\left\{U+\alpha Z, U^{\prime}+\alpha^{\prime} Z\right\}$ be a two-dimensional subalgebra of $\mathfrak{h}^{1}$, where $U, U^{\prime}$ belong to the first layer $\mathfrak{v}$. If $\left[U, U^{\prime}\right]=0$ then they are proportional and $\mathfrak{a} \oplus \mathfrak{n}$ is two-dimensional. It follows that $\left[U, U^{\prime}\right]=\gamma Z$, with $\gamma \neq 0$. On the other hand, $a$ is a subalgebra, and hence $Z \in \mathfrak{a}$ and this would imply $\mathfrak{a}=\mathfrak{h}^{1}$. This is also a contradiction, so such a complementary subalgebra cannot exist.

REMARK. All the homogeneous subgroups $\exp \mathfrak{a}_{\lambda}=\exp \operatorname{span}\{X+\lambda Y\}$ of $\mathbb{H}^{1}$ with $\lambda \in \mathbb{R}$ yield complementary subgroups $A_{\lambda}=\exp \mathfrak{a}_{\lambda}$ of $S=\exp (\operatorname{span}\{Y, Z\})$. Hence, complementary subgroups, when they exist, need not be unique.

Next, we wish to see how the notion of complementary subgroup is translated for the corresponding subalgebras, when $\mathbb{G}$ is a graded group. First of all, we notice that a decomposition of $\mathcal{G}$ as a direct sum of two subspaces does not ensure a corresponding decomposition of $\mathbb{G}$ as the product of their images through the exponential mapping. This simple fact is shown in the next examples.

EXAmple 7.8. We consider the Heisenberg algebra $\mathfrak{h}^{1}$ with basis $(X, Y, Z)$ and bracket relation $[X, Y]=Z$. We consider the following subspaces of $\mathfrak{h}^{1}$ :

$$
\left\{\begin{array}{l}
\mathfrak{u}=\operatorname{span}\{X, Y\}, \\
\mathfrak{w}=\operatorname{span}\left\{Y+\frac{1}{2} Z\right\} .
\end{array}\right.
$$

It is immediate to check that $\mathfrak{u} \oplus \mathfrak{w}=\mathfrak{h}^{1}$, but $\exp \mathfrak{u} \exp \mathfrak{w} \neq \mathbb{H}^{1}$, since for every $\lambda \neq 0$ we have $\exp (-X+\lambda Z) \notin \exp \mathfrak{u} \exp \mathfrak{w}$.

EXAMPLE 7.9. We consider the Heisenberg algebra $\mathfrak{h}^{2}$ equipped with the basis $\left(X_{1}, Y_{1}, X_{2}, Y_{2}, Z\right)$ and bracket relations $\left[X_{1}, Y_{1}\right]=\left[X_{2}, Y_{2}\right]=Z$. Then we define the linear subspaces

$$
\left\{\begin{array}{l}
\mathfrak{a}=\operatorname{span}\left\{X_{1}, X_{2}, Z+Y_{1}\right\}, \\
\mathfrak{b}=\operatorname{span}\left\{Y_{1}, Y_{2}\right\}
\end{array}\right.
$$

It is immediate to check that $\mathfrak{a} \oplus \mathfrak{b}=\mathfrak{h}^{2}$, but $\exp \left(2 X_{1}+Z\right) \notin A B$, where $A=\exp \mathfrak{a}$ and $B=\exp \mathfrak{b}$.

7.2. $\boldsymbol{h}$-epimorphisms and $\boldsymbol{h}$-monomorphisms. In this subsection we use complementary subgroups to characterize both $h$-epimorphisms and $h$-monomorphisms. These characterizations will be used in the proof of both the implicit function theorem and the rank theorem. 
Proposition 7.10 (Characterization of $h$-epimorphisms). We consider a surjective h-homomorphism $L: \mathbb{G} \longrightarrow \mathbb{M}$ and denote its kernel by $N$. Then the following conditions are equivalent:

(1) there exists a subgroup $H$ complementary to $N$;

(2) L is an h-epimorphism.

Moreover, if one of them holds, then the restriction $L_{\mid H}$ is an h-isomorphism.

Proof. We first assume the validity of the first condition. Then we consider the restriction $T=L_{\mid H}: H \longrightarrow \mathbb{M}$. Let $m \in \mathbb{M}$ and let $g \in \mathbb{G}$ such that $L(g)=m$. Then the property $N H=\mathbb{G}$ implies that $g$ is $n h$, where $(n, h) \in N \times H$. As a consequence, we have $L(n h)=L(h)=T(h)=m$, and hence $T$ is surjective. If we have $T(h)=e$, then $h \in H \cap N=\{e\}$. We have shown that $L_{\mid H}$ is an $h$-homomorphism. Clearly, $T^{-1}: \mathbb{M} \longrightarrow H$ is an $h$-homomorphism and satisfies $L \circ T^{-1}=\operatorname{Id}_{\mathbb{M}}$, so $L$ is an $h$-epimorphism. Conversely, if $L$ is an $h$-epimorphism, then there exists a right inverse $R: \mathbb{M} \longrightarrow \mathbb{G}$ that is also an $h$-homomorphism. We set $H=R(\mathbb{M})$ and easily observe that $H \cap N=\{e\}$. Let $g \in \mathbb{G}$ and consider $m=L(g)=L(R(m))$, so that $g^{-1} R(m) \in N$, and this implies that $g \in H N$. Then $\mathbb{G}=H N=N H$, and this concludes the proof.

Proposition 7.11 (Characterization of $h$-monomorphisms). Let us consider an injective h-homomorphism $T: \mathbb{G} \longrightarrow \mathbb{M}$, along with its image $H$. The following conditions are equivalent:

(1) there exists a normal subgroup $N$ complementary to $H$;

(2) there exists an h-epimorphism $p: \mathbb{M} \longrightarrow H$ such that $p_{\mid H}=\operatorname{Id}_{H}$;

(3) $T$ is an h-monomorphism.

Proof. We show that (1) implies (2). We define the projection $p: \mathbb{M} \longrightarrow H$ that associates to any element $m=n h \in \mathbb{M}$, with $(n, h) \in N \times H$, the element $h \in H$. This definition is well posed, since $N$ and $H$ are complementary. The fact that $N$ is normal and the uniqueness of representation of the product $n h$ give

$$
p\left(\left(n_{1} h_{1}\right)\left(n_{2} h_{2}\right)\right)=p\left(\left(n_{1} h_{1} n_{2} h_{1}^{-1}\right)\left(h_{1} h_{2}\right)\right)=h_{1} h_{2}=p\left(n_{1} h_{1}\right) p\left(n_{2} h_{2}\right) .
$$

It is trivial to observe that $p$ is homogeneous and that its restriction to $H$ is exactly $\operatorname{Id}_{H}$. Then $p$ is a surjective $h$-homomorphism. Furthermore, $H$ is a complementary subgroup of the kernel $N$, so Proposition 7.10 implies that $p$ is an $h$-epimorphism.

To prove that (2) implies (3), we define the mapping $L=J \circ p$, where the injectivity of $T$ allows us to define the $h$-isomorphism $J: H \longrightarrow \mathbb{G}$ such that $J(T(g))=g$ for every $g \in \mathbb{G}$. Then $L$ is an $h$-homomorphism as composition of $h$-homomorphisms. In addition, one can easily verify that $L \circ T=\operatorname{Id}_{\mathbb{G}}$.

We are left to show that (3) implies (1). By definition of $h$-monomorphism, we have an $h$-homomorphism $L: \mathbb{M} \longrightarrow \mathbb{G}$ that is a left inverse of $T$. Let $N$ be its kernel, that is a normal homogeneous subgroup of $\mathbb{M}$. We have to show that $N$ and $H$ are complementary subgroups. Let $m \in \mathbb{M}$ and consider $L(m)=g \in \mathbb{G}$. We have

$$
L\left(m T\left(g^{-1}\right)\right)=L(m) g^{-1}=e,
$$


and hence $m T\left(g^{-1}\right)=n \in N$, that is, $m=n T(g) \in N H$. Let $x \in N \cap H$ and let $g \in \mathbb{G}$ such that $x=T(g)$. Thus, we get $e_{\mathbb{G}}=L(x)=L \circ T(g)=g$, which implies $g=e_{\mathbb{G}}$ and $x=T\left(e_{\mathbb{G}}\right)=e_{\mathbb{M}}$, that is, $N \cap H=\left\{e_{\mathbb{M}}\right\}$.

\section{Quotients of graded groups}

In this section we show that the group quotient of graded groups is still graded with a natural left invariant and homogeneous distance. We first recall some elementary facts of Lie groups theory in order to study the relationship between quotients of Lie algebras and quotients of Lie groups.

Let $G$ be a real Lie group and let $H$ be a Lie subgroup of $G$. Recall that the quotient $G / H$ has a unique manifold structure that makes the projection $\pi: G \longrightarrow G / H$ a smooth mapping. $G / H$ is called a homogeneous manifold, see [30, Theorem 3.58].

If we consider a normal Lie subgroup $N$, then $G / N$ is, in addition, a Lie group, according to [30, Theorem 3.64], and in this case

$$
\pi: G \longrightarrow G / N
$$

is clearly is a Lie group homomorphism. As a result, by [30, Theorem 3.14],

$$
d \pi: \mathcal{G} \longrightarrow \overline{\mathcal{G}}
$$

is a Lie algebra homomorphism, where $\mathcal{G}$ and $\overline{\mathcal{G}}$ are the Lie algebras of $G$ and $G / N$, respectively.

Remark. The mapping $d \pi$ is also surjective. In fact, [30, Theorem 3.58] ensures the existence of a neighborhood $W \subset G / N$ of the unit element $\bar{e}$ of $G / N$ and a smooth mapping $\tau: W \longrightarrow G$ such that $\pi \circ \tau=\operatorname{Id}_{W}$. Let $\Gamma:(-\varepsilon, \varepsilon) \longrightarrow W$ be a smooth curve with $\dot{\Gamma}(0)=\bar{X}_{\bar{e}} \in T_{\bar{e}}(G / N)$. Then the curve $\gamma=\tau \circ \Gamma:(-\varepsilon, \varepsilon) \longrightarrow G$ satisfies $\pi \circ \gamma=\Gamma$ and $d \pi\left(X_{e}\right)=\bar{X}_{\bar{e}}$, where $X_{e}=\dot{\gamma}(0)$. This shows surjectivity of $d \pi$.

Proposition 8.1. The Lie algebra $\overline{\mathcal{G}}$ of $G / N$ is isomophic to the quotient algebra $\mathcal{G} / \mathcal{N}$, where $\mathcal{G}$ is the Lie algebra of $G$ and $\mathcal{N}$ is the ideal corresponding to the normal subgroup $N$. Furthermore, we can represent the exponential mapping of $G / N$ on $G / N$ by setting $\operatorname{Exp}: \mathcal{G} / \mathcal{N} \longrightarrow G / N$,

$$
\operatorname{Exp}(X+\mathcal{N})=\pi(\exp (X))
$$

where $\exp : \mathcal{G} \longrightarrow G$ is the exponential mapping of $G, \pi: G \longrightarrow G / N$ is the canonical projection and the diagram

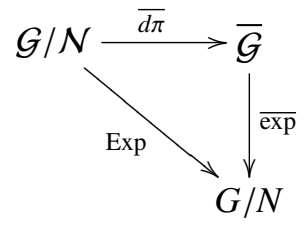

commutes, where $\overline{\exp }: \overline{\mathcal{G}} \longrightarrow G / N$ is the canonical exponential mapping of $G / N$. 
Proof. We have seen above that $d \pi: \mathcal{G} \longrightarrow \overline{\mathcal{G}}$ is surjective, hence

$$
\operatorname{dim}(\operatorname{ker}(d \pi))=\operatorname{dim}(\mathcal{N}) .
$$

For every $U \in \mathcal{N}$ the curve $\pi(\exp (t X))$ is constantly equal to the unit element $\bar{e}$ of $G / N$, then $d \pi(X)=0$ and clearly $\mathcal{N} \subset \operatorname{ker} d \pi$. It follows that $\mathcal{N}=\operatorname{ker} d \pi$ and the algebra isomorphism $\overline{d \pi}: \mathcal{G} / \mathcal{N} \longrightarrow \overline{\mathcal{G}}$ is well defined, where $p: \mathcal{G} \longrightarrow \mathcal{G} / \mathcal{N}$ is the canonical projection and

$$
[p(X), p(Y)]=p([X, Y])
$$

for every $X, Y \in \mathcal{G}$. If we consider the diagram

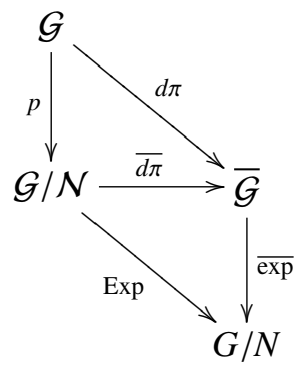

then we are left to show that its lower part commutes. In fact, by definition of $\overline{d \pi}$, for every $X \in \mathcal{G}$

$$
\overline{\exp } \circ \overline{d \pi}(p(X))=\overline{\exp } \circ d \pi(X) .
$$

On the other hand, due to [30, Theorem 3.32], the diagram

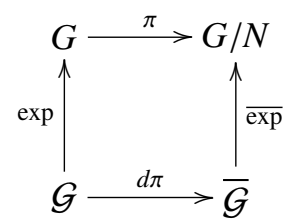

commutes, namely $\overline{\exp } \circ d \pi(X)=\pi \circ \exp (X)$ for every $X \in \mathcal{G}$. It follows that

$$
\overline{\exp } \circ \overline{d \pi}=\pi \circ \exp =\operatorname{Exp} .
$$

This concludes the proof.

Proposition 8.2. Let $\mathbb{M}$ be a graded group and let $\mathcal{M}=W_{1} \oplus \cdots \oplus W_{v}$ be its Lie algebra. Let $N$ be a normal homogeneous subgroup corresponding to the ideal $\mathcal{N}=\mathcal{N}_{1} \oplus \cdots \oplus \mathcal{N}_{v}$. Then the surjective Lie algebra homomorphism $d \pi: \mathcal{M} \longrightarrow \overline{\mathcal{M}}$ induces a grading

$$
\bar{W}_{1} \oplus \cdots \oplus \bar{W}_{v}
$$


on the Lie algebra $\overline{\mathcal{M}}$ of $\mathbb{M} / N$, which is h-isomorphic to

$$
W_{1} / \mathcal{N}_{1} \oplus \cdots \oplus W_{v} / \mathcal{N}_{v}
$$

where $d \pi\left(W_{i}\right)=\bar{W}_{i}$ is linearly isomorphic to $W_{i} / \mathcal{N}_{i}$. Moreover, a family of dilations $\bar{\delta}_{r}$ can be defined on $\mathbb{M} / N$ such that $\pi: \mathbb{M} \longrightarrow \mathbb{M} / N$ is an h-homomorphism. Finally, if $\mathbb{M}$ is stratified then so is $\mathbb{M} / N$.

Proof. The decomposition of $\mathcal{N}$ into factors $\mathcal{N}_{i}$ follows from Corollary 7.3, where some of the $\mathcal{N}_{i}$ are possibly vanishing. By Proposition 8.1 the Lie algebra $\overline{\mathcal{M}}$ of the quotient $\mathbb{M} / N$ is isomorphic to $\mathcal{M} / \mathcal{N}$ and

$$
p: \mathcal{M} \longrightarrow \mathcal{M} / \mathcal{N} \text { is equivalent to } d \pi: \mathcal{M} \longrightarrow \overline{\mathcal{M}},
$$

by commutativity of the diagram (8.1), and hence we consider the grading induced by $p$. Let us check that $p\left(W_{i}\right) \cap p\left(W_{j}\right)=\{0\}$ whenever $i \neq j$. By contradiction, let $\bar{X} \in$ $p\left(W_{i}\right) \cap p\left(W_{j}\right)$ be nonvanishing. Then there exist $X_{i} \in W_{i} \backslash \mathcal{N}$ and $X_{j} \in W_{j} \backslash \mathcal{N}$ such that $X_{i}=X_{j}+Z$, where $Z \in \mathcal{N}$. Since $\mathcal{N}$ is homogeneous, we must have $Z=Z_{i}+Z_{j}$, where $Z_{i} \in W_{i} \cap \mathcal{N}$ and $Z_{j} \in W_{j} \cap \mathcal{N}$, and hence $Z_{i}=X_{i}$ and $Z_{j}=-X_{j}$, which conflicts with our initial assumption on $X_{i}$ and $X_{j}$. This shows that $\overline{\mathcal{M}}=\bar{W}_{1} \oplus \cdots \oplus \bar{W}_{v}$ is a grading, where $\bar{W}_{i}=d \pi\left(W_{i}\right)$. This immediately implies that $\overline{\mathcal{M}}$ is stratified when $\mathcal{M}$ is stratified. If we set $\bar{\delta}_{r}\left(\bar{X}_{i}\right)=r^{i} \bar{X}_{i}$ for every $\bar{X}_{i} \in \bar{W}_{i}$, then one can easily check that $d \pi \circ \delta_{r}=\bar{\delta}_{r} \circ d \pi$ and $\pi$ is an $h$-homomorphism. Finally, we consider the commutative diagram

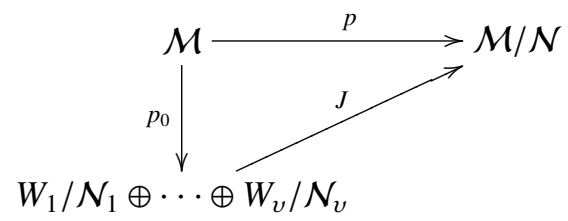

where we have set $p_{0}\left(w_{1}+\cdots+w_{v}\right)=\left(w_{1}+\mathcal{N}_{1}, \ldots, w_{v}+\mathcal{N}_{v}\right)$ and the definition $J\left(w_{1}+\mathcal{N}_{1}, \ldots, w_{v}+\mathcal{N}_{v}\right)=w_{1}+\cdots+w_{v}+\mathcal{N}$ is well posed. It is immediate to observe that ker $J=\{0\}$, so $J$ is a linear isomorphism. The commutativity of the diagram above implies that $p\left(W_{i}\right)=J\left(W_{i} / \mathcal{N}_{i}\right)$. This concludes the proof.

REMARK. The induced grading on $\mathcal{M} / \mathcal{N}$ considered in Proposition 8.2 is given by the subspaces $\bar{W}_{j}=d \pi\left(W_{j}\right)$, which satisfy $\mathcal{M} / \mathcal{N}=\bar{W}_{1} \oplus \cdots \oplus \bar{W}_{v}$, where some of these factors are possibly vanishing. On the other hand, when $\mathcal{N}$ is not homogeneous $\mathcal{M} / \mathcal{N}$ still can have a grading with dilations, but these ones do not commute with $\pi$, that is, $\pi$ is no longer an h-homomorphism.

ExAmple 8.3. Let $W_{1}=\operatorname{span}\left\{X_{1}, X_{2}, X_{3}\right\}$ and $W_{2}=\operatorname{span}\{Z\}$, where the only nontrivial bracket relation is $\left[X_{1}, X_{2}\right]=Z$. Then $\mathcal{M}=W_{1} \oplus W_{2}$ is a 2-step stratified algebra and $\mathcal{N}=\operatorname{span}\left\{X_{3}-Z\right\}$ is an ideal of $\mathcal{M}$ that is clearly not homogeneous. Elements of $\mathcal{M} / \mathcal{N}$ are $\bar{X}_{i}=X_{i}+\mathcal{N}$, where $i=1,2,3$ and $\bar{X}_{3}=Z+\mathcal{N}$. Clearly, the only nontrivial bracket relation is $\left[\bar{X}_{1}, \bar{X}_{2}\right]=\bar{X}_{3}$ and $\mathcal{M} / \mathcal{N}$ is isomorphic to the three-dimensional Heisenberg 
algebra. On the other hand, there are no dilations $\bar{\delta}_{r}$ on $\mathcal{M} / \mathcal{N}$ such that $d \pi \circ \delta_{r}=\bar{\delta}_{r}$ 。 $d \pi$, since $d \pi\left(\delta_{r} X_{3}\right)=r d \pi\left(X_{3}\right)=r \bar{X}_{3}$ and $d \pi\left(\delta_{r} Z\right)=r^{2} d \pi(Z)=r^{2} \bar{X}_{3}$. We also notice that in this case $d \pi$ cannot induce any grading on $\overline{\mathcal{M}}$, since $d \pi\left(W_{1}\right) \cap d \pi\left(W_{2}\right)=\left\{\bar{X}_{3}\right\}$.

\section{Implicit function theorem and rank theorem}

In this section we prove both the implicit function theorem and the rank theorem stated in the introduction. We will use the notation $D_{\bar{n}, r}^{N}=D_{\bar{n}, r} \cap N$ and $D_{\bar{h}, s}^{H}=$ $D_{\bar{h}, s} \cap H$.

Next, we give the proof of Theorem 1.4.

Proof. Our arguments are divided into two main steps.

Step 1. Existence. By Proposition 7.10, the restriction $D f(\bar{x})(h): H \longrightarrow \mathbb{G}$ is invertible, and

$$
\min _{\substack{d(h)=1 \\ h \in H}} \rho(D f(\bar{x})(h))>0 .
$$

By continuity of $\Omega \times H \ni(x, h) \longrightarrow \rho(D f(x)(h))$, there exists $R>0$ such that

$$
\mu=\min _{x \in D_{\bar{x}, R}} \min _{\substack{d(h)=1 \\ h \in H}} \rho(D f(x)(h))>0 \quad \text { and } \quad \bar{\mu}=\max _{x \in D_{\bar{x}, R}} \max _{\substack{d(h)=1 \\ h \in H}} \rho(D f(x)(h))>0
$$

with $D_{\bar{x}, R} \subset \Omega$. Now we set $R_{0}=R /(2+2 c(\mathbb{G}, d) N)$, where $c(\mathbb{G}, d)$ and $N$ are as in (4.10) and Lemma 4.9, respectively. Then condition (1.5) is satisfied and Theorem 1.2 yields the estimate

$$
\max _{x, y \in D_{\bar{x}, R_{0}}} \rho\left(f(x)^{-1} f(y), D f(x)\left(x^{-1} y\right)\right) \leq C\left(\omega_{D_{\bar{x}, R}, d_{H} F_{1}}(N c d(x, y))\right)^{1 / \iota^{2}} d(x, y) .
$$

Thus, by definition of $\bar{\mu}$ in (9.1),

$$
\rho(f(x), f(y)) \leq\left(C\left[\omega_{D_{\bar{x}, R}, d_{H} F_{1}}(N c d(x, y))\right]^{1 / \iota^{2}}+\bar{\mu}\right) d(x, y)
$$

for every $x, y \in D_{\bar{x}, R_{0}}$. Let $(\bar{n}, \bar{h})$ be the unique tuple in $N \times H$ such that $\bar{n} \bar{h}=\bar{x}$ and let $r, s>0$ be such that $D_{\bar{n}, r}^{N} D_{\bar{h}, s}^{H} \subset D_{\bar{x}, R_{0}}$. Then, using the definition of $\mu$ in (9.1), for every $n \in D_{\bar{n}, r}^{N}$ and every $h, h^{\prime} \in D_{\bar{h}, s}^{H}$, we have the inequality

$$
\rho\left(f(n h), f\left(n h^{\prime}\right)\right) \geq\left(\mu-C\left[\omega_{D_{\bar{x}, R}, d_{H} F_{1}}\left(N c d\left(h, h^{\prime}\right)\right)\right]^{1 / \iota^{2}}\right) d\left(h, h^{\prime}\right),
$$

since $d\left(n h, n h^{\prime}\right)=d\left(h, h^{\prime}\right)$. Using (9.3) and (9.4) and possibly taking a smaller $s>0$, depending on $C, N, c>0$, we get a constant $\beta>0$ such that

$$
\beta^{-1} d\left(h, h^{\prime}\right) \leq \rho\left(F_{n}(h), F_{n}\left(h^{\prime}\right)\right) \leq \beta d\left(h, h^{\prime}\right),
$$


where for each $n \in D_{\bar{n}, r}^{N}$, we have defined $F_{n}: D_{\bar{h}, s}^{H} \longrightarrow \mathbb{M}$, with $F_{n}(h)=f(n h)$. Observing that $F_{\bar{n}}(\bar{h})=f(\bar{x})$ and taking into account the bi-Lipschitz estimate (9.5), we have $F_{\bar{n}}(h) \neq f(\bar{x})$ for every $h \in \partial D_{\bar{h}, s}^{H}$. Injectivity of $F_{\bar{n}}$ implies that

$$
\operatorname{deg}\left(F_{\bar{n}}, B_{\bar{h}, s}^{H}, f(\bar{x})\right) \in\{-1,1\},
$$

see, for instance, [14, Theorem 3.3.3]. Since the function

$$
n \longrightarrow \max _{h \in \partial D_{\bar{h}, s}^{H}} \rho(f(n h), f(\bar{n} h))
$$

is continuous, up to choosing a smaller $r>0$, we can assume that

$$
\max _{h \in \partial D_{\bar{h}, s}^{H}} \rho(f(n h), f(\bar{n} h))<\frac{s}{2 \beta}
$$

for every $n \in D_{\bar{n}, r}^{N}$. As a consequence, applying (9.5), it follows that

$$
\rho\left(F_{n}(h), f(\bar{x})\right) \geq \rho\left(F_{\bar{n}}(h), f(\bar{x})\right)-\rho\left(F_{\bar{n}}(h), F_{n}(h)\right)>\frac{s}{2 \beta}
$$

for every $h \in \partial D_{\bar{h}, s}^{H}$ and every $n \in D_{\bar{n}, r}^{N}$. For an arbitrary $n \in D_{\bar{n}, r}^{N} \backslash\{\bar{n}\}$ one can consider the continuous curve $\gamma:[0,1] \longrightarrow D_{\bar{n}, r}^{N}$, defined by $\gamma(t)=\bar{n} \delta_{t}\left((\bar{n})^{-1} n\right)$. Notice that $\gamma$ has image in $D_{\bar{n}, r}^{N}$, since $N$ is a homogeneous subgroup of $\mathbb{G}$. By previous estimates, the mapping $\Phi:[0,1] \times D_{\bar{h}, s} \longrightarrow \mathbb{M}$ defined by $\Phi(t, h)=f(\gamma(t) h)$ is a homotopy between $F_{\bar{n}}$ and $F_{n}$ such that $\Phi(t, h) \neq f(\bar{x})$ for every $t \in[0,1]$ and every $h \in \partial D_{\bar{h}, s}^{H}$. Thus, homotopy invariance of topological degree [26], implies

$$
\operatorname{deg}\left(F_{n}, B_{\bar{h}, s}^{H}, f(\bar{x})\right)=\operatorname{deg}\left(F_{\bar{n}}, B_{\bar{h}, s}^{H}, f(\bar{x})\right) \neq 0,
$$

and hence there exists at least one element $h^{\prime} \in D_{\bar{h}, s}^{H}$, depending on $n$, such that $F_{n}\left(h^{\prime}\right)=f(\bar{x})$. Injectivity of $F_{n}$ gives uniqueness of $h^{\prime}$, so there exists $\varphi: D_{\bar{n}, r}^{N} \longrightarrow D_{\bar{h}, s}^{H}$, uniquely defined, such that $F_{n}(\varphi(n))=f(n \varphi(n))=f(\bar{x})$.

Step 2. Regularity. We keep the same notation of the previous step. By definition of $\mu>0$ in (9.1), the restriction $L(x)=(D f(x))_{\mid H}: H \longrightarrow \mathbb{M}$ is invertible for every $x \in D_{\bar{x}, R_{0}}$ and setting $T(x)=L(x)^{-1}$,

$$
d(T(x)(m)) \leq \mu^{-1} \rho(m)
$$

for every $m \in \mathbb{M}$. Due to (9.2), we can choose a possibly smaller $R>0$, and hence a smaller $R_{0}=R /(2+2 c N)$, such that

$$
\max _{x, y \in D_{\bar{x}, R_{0}}} \rho\left(f(x)^{-1} f(y), D f(x)\left(x^{-1} y\right)\right) \leq \frac{\mu d(x, y)}{2} .
$$


It follows that the remainder $E\left(n, n^{\prime}\right)$ in the expression

$$
f(n \varphi(n))^{-1} f\left(n \varphi\left(n^{\prime}\right)\right)=L(n \varphi(n))\left(\varphi(n)^{-1} \varphi\left(n^{\prime}\right)\right) E\left(n, n^{\prime}\right)
$$

satisfies the uniform estimate

$$
\rho\left(E\left(n, n^{\prime}\right)\right) \leq \frac{\mu}{2} d\left(\varphi(n), \varphi\left(n^{\prime}\right)\right)
$$

for every $n, n^{\prime} \in D_{\bar{n}, r}^{N}$. The implicit mapping $\varphi$ satisfies

$$
f(n \varphi(n))^{-1} f\left(n \varphi\left(n^{\prime}\right)\right)=\left(f\left(n \varphi\left(n^{\prime}\right)\right)^{-1} f\left(n^{\prime} \varphi\left(n^{\prime}\right)\right)\right)^{-1},
$$

and therefore one easily gets

$$
\varphi(n)^{-1} \varphi\left(n^{\prime}\right)=T(n \varphi(n))\left(f\left(n \varphi\left(n^{\prime}\right)\right)^{-1} f\left(n^{\prime} \varphi\left(n^{\prime}\right)\right)\right)^{-1} T(n \varphi(n))\left(E\left(n, n^{\prime}\right)\right)^{-1} .
$$

As a result, in view of (9.6) and (9.7),

$$
d\left(\varphi(n), \varphi\left(n^{\prime}\right)\right) \leq \frac{2}{\mu} \rho\left(f\left(n \varphi\left(n^{\prime}\right)\right), f\left(n^{\prime} \varphi\left(n^{\prime}\right)\right)\right) .
$$

By (9.3), up to choosing a possibly smaller $R>0$, we can suppose that

$$
\rho(f(x), f(y)) \leq 2 \bar{\mu} d(x, y)
$$

for every $x, y \in D_{\bar{x}, R_{0}}$, and hence

$$
d\left(\varphi(n), \varphi\left(n^{\prime}\right)\right) \leq \frac{4 \bar{\mu}}{\mu} d\left(\varphi\left(n^{\prime}\right)^{-1} n^{-1} n^{\prime} \varphi\left(n^{\prime}\right)\right) .
$$

Finally, formulas (3.13) and (3.10) lead us to the conclusion.

REMARK. Under the hypotheses of Theorem 1.4, one immediately gets

$$
d\left(n \varphi(n), n^{\prime} \varphi\left(n^{\prime}\right)\right) \leq(1+\kappa) d\left(\varphi\left(n^{\prime}\right)^{-1} n^{-1} n^{\prime} \varphi\left(n^{\prime}\right)\right),
$$

Proof of Theorem 1.5. By Proposition 7.11, we have a projection $p: \mathbb{M} \longrightarrow H$ that is an $h$-epimorphism and $p_{\mid H}=\mathrm{Id}_{H}$. We also know that the kernel $N$ of $p$ is complementary to $H$. As a first consequence, the mapping $p \circ f: \Omega \longrightarrow H$ is continuously differentiable and $D(p \circ f)(\bar{x}): \mathbb{G} \longrightarrow H$ is invertible. Due to Theorem 1.3, there exist open neighborhoods $V \Subset \Omega$ and $W \Subset H$ of $\bar{x}$ and $p(f(\bar{x}))$, respectively, such that the restriction $(p \circ f)_{\mid \bar{V}}: \bar{V} \longrightarrow \bar{W}$ is invertible. We denote by $\psi: \bar{W} \longrightarrow \bar{V}$ its inverse function. By the second remark of Section 7.1, there exists a unique function $f_{N}: \Omega \longrightarrow N$ such that $f(x)=p(f(x)) f_{N}(x)$ for every $x \in \Omega$.

Then we consider $g: \bar{W} \longrightarrow \mathbb{M}$ defined by $g(h)=f(\psi(h))=h f_{N}(\psi(h))$ for every $h \in \bar{W}$. The uniqueness of the factorization implies that $g\left(W^{\prime}\right)=g\left(W^{\prime}\right) \cap W^{\prime} N$, whenever $W^{\prime} \subset \bar{W}$. If $W^{\prime}$ is open, then so is $W^{\prime} N$, since the mapping (7.1) is open. 
As a consequence, $g$ is an open mapping on its image, and hence so is the restriction $f_{\mid V}$. This implies that $f_{\mid V}$ is a topological embedding. If we set

$$
\varphi=f_{N} \circ \psi \quad \text { and } \quad \Psi=I \circ J^{-1} \circ \psi \circ p_{\mid f(V)}
$$

then $\varphi(h)=h^{-1} g(h)$ and (1.9) holds, where $I$ and $J$ are defined in the statement of Theorem 1.5. We notice that $g$ is continuously differentiable on $\bar{W}$, and therefore, in particular, it is Lipschitz on $\bar{W}$ up to a suitable choice of $W$, for instance, a closed ball. Our next computations are performed in the Lie algebra $\mathcal{G}$ of $\mathbb{G}$. We set $\varphi=\exp \circ F$ and $g=\exp \circ G$, and therefore Lemma 3.5 gives a constant $C>0$, depending on both $\bar{W}$ and the Lipschitz constant of $g$, such that

$$
\left\|G(h)-G\left(h^{\prime}\right)\right\| \leq C d\left(h, h^{\prime}\right) \quad \text { and } \quad\left\|\xi-\xi^{\prime}\right\| \leq C d\left(h, h^{\prime}\right),
$$

where $h=\exp \xi$ and $h^{\prime}=\exp \xi^{\prime}$, with $h, h^{\prime} \in \bar{W}$. Then

$$
\begin{aligned}
F(h) & -F\left(h^{\prime}\right)=(-\xi) \odot G(h)-\left(\left(-\xi^{\prime}\right) \odot G\left(h^{\prime}\right)\right) \\
& =\xi^{\prime}-\xi+G(h)-G\left(h^{\prime}\right)+\sum_{n=2}^{\iota}\left[c_{n}(-\xi, G(h))-c_{n}\left(-\xi^{\prime}, G\left(h^{\prime}\right)\right)\right],
\end{aligned}
$$

due to the formula (2.2). From (3.20), it follows that

$$
\left\|F(h)-F\left(h^{\prime}\right)\right\| \leq\left\|\xi^{\prime}-\xi\right\|+\left\|G(h)-G\left(h^{\prime}\right)\right\|+\sum_{n=2}^{\iota} \gamma_{n} v^{n-1}\left(\left\|\xi-\xi^{\prime}\right\|+\left\|G(h)-G\left(h^{\prime}\right)\right\|\right),
$$

where we have set $v=2\left(\max _{\eta \in \bar{W}}\|G(\eta)\|+\sup _{\eta \in \bar{W}}\|\eta\|\right)$. By virtue of (9.9), we get a constant $C_{1}>0$, depending on $\bar{W}, v$ and the Lipschitz constant of $g$, such that $\left\|F(h)-F\left(h^{\prime}\right)\right\| \leq C_{1} d\left(h, h^{\prime}\right)$. This concludes the proof.

\section{Intrinsic graphs and $(\mathbb{G}, \mathbb{M})$-regular sets}

The notion of complementary subgroup allows us to set properly the wellestablished notion of intrinsic graph in those graded groups that admit a factorization into two complementary subgroups.

Definition 10.1. Let $P$ and $H$ be complementary subgroups of $\mathbb{G}$ and let $S \subset \mathbb{G}$. We say that $S$ is an intrinsic graph with respect to $(P, H)$ if there exists a subset $A \subset P$ and a mapping $\varphi: A \longrightarrow H$ such that $S=\{p \varphi(p) \mid p \in A\}$.

REMARK (Translations of intrinsic graphs). As already observed in [7], intrinsic graphs are preserved under left translations. If $P$ and $H$ are complementary subgroups of $\mathbb{G}$ and $S=\{p \varphi(p) \mid p \in A\}$ is an intrinsic graph with respect to $(P, H)$, then the left translated $x p \varphi(p)$, for some $x \in \mathbb{G}$, is of the form $\tilde{p} \psi(\tilde{p})$, where $\tilde{p} \in \tilde{A} \subset P$ and $\psi: \tilde{A} \longrightarrow H$. In fact, by unique decomposition of elements in $\mathbb{G}$ with respect to $P$ and $H$, there exist functions $\psi_{1}: A \longrightarrow P$ and $\psi_{2}: A \longrightarrow H$ such that

$$
x p \varphi(p)=\psi_{1}(p) \psi_{2}(p) .
$$


To conclude, it suffices to show that $\psi_{1}$ is injective. If $\psi_{1}(p)=\psi_{1}\left(p^{\prime}\right)$, then

$$
x p \varphi(p) \psi_{2}(p)^{-1}=x p^{\prime} \varphi\left(p^{\prime}\right) \psi_{2}\left(p^{\prime}\right)^{-1},
$$

where $\varphi(p) \psi_{2}(p)^{-1}, \varphi\left(p^{\prime}\right) \psi_{2}\left(p^{\prime}\right)^{-1} \in H$. Again, uniqueness yields $p=p^{\prime}$.

Definition $10.2((\mathbb{G}, \mathbb{M})$-regular set of $\mathbb{G})$. Let $\mathbb{G}$ be a stratified group and let $\mathbb{M}$ be a graded group such that $\mathbb{M}$ is an $h$-quotient of $\mathbb{G}$. We say that a subset $S \subset \mathbb{G}$ is $(\mathbb{G}, \mathbb{M})$-regular if for every point $\bar{x} \in S$, there exists an open neighborhood $U$ of $\bar{x}$ and a continuously differentiable mapping $f: U \longrightarrow \mathbb{M}$ such that $S \cap U=f^{-1}(e)$ and $D f(x): \mathbb{G} \longrightarrow \mathbb{M}$ is an $h$-epimorphism for every $x \in U$.

Definition $10.3((\mathbb{G}, \mathbb{M})$-regular set of $\mathbb{M})$. Let $\mathbb{G}$ be a stratified group and let $\mathbb{M}$ be a graded group such that $\mathbb{G} h$-embeds into $\mathbb{M}$. A subset $S \subset \mathbb{M}$ is $(\mathbb{G}, \mathbb{M})$-regular if for every point $\bar{x} \in S$, there exist open neighborhoods $U \subset \mathbb{M}$ of $\bar{x}$ and $V \subset \mathbb{G}$ of $e \in \mathbb{G}$, along with a continuously differentiable topological embedding $f: V \longrightarrow \mathbb{M}$, such that $S \cap U=f(V)$ and $D f(y): \mathbb{G} \longrightarrow \mathbb{M}$ is an $h$-monomorphism for every $y \in V$.

Definition $10.4((\mathbb{G}, \mathbb{M})$-regular set). When a subset $S$ is either $(\mathbb{G}, \mathbb{M})$-regular in $\mathbb{G}$ or $(\mathbb{G}, \mathbb{M})$-regular in $\mathbb{M}$, we simply say that it is $(\mathbb{G}, \mathbb{M})$-regular without further specification, or we can say that it is an intrinsically regular set.

10.1. Tangent cone to $(\mathbb{G}, \mathbb{M})$-regular sets. The notion of 'tangent cone' given in $[3,3.1 .21]$ can be easily extended to graded groups.

Definition 10.5. Let $\mathbb{G}$ be a graded group and let $S \subset \mathbb{G}$ with $a \in \mathbb{G}$. The homogeneous tangent cone of $S$ at $a$ is the homogeneous subset

$$
\operatorname{Tan}(S, a)=\left\{v \in \mathbb{G} \mid \delta_{r_{k}}\left(a^{-1} s_{k}\right) \rightarrow v,\left(r_{k}\right) \subset \mathbb{R}^{+},\left(s_{k}\right) \subset S \text { and } s_{k} \rightarrow a\right\} .
$$

REMARK. If $a \in \bar{S}$, then $\operatorname{Tan}(S, a) \neq \emptyset$.

Proof of Theorem 1.7. Replacing $f$ with $f \circ l_{x}$, where $l_{x}(y)=x y$, it is not restrictive to assume that $\bar{x}=e$, since

$$
(\bar{x})^{-1} S=\left(f \circ l_{\bar{x}}\right)^{-1}\left(f \circ l_{\bar{x}}\right)(e)
$$

and $D f(\bar{x})=D\left(f \circ l_{\bar{x}}\right)(e)$. Now we use the notation of Theorem 1.4, applying it in the case $\bar{x}=e$. From differentiability of $f$ at $e$ and the representation (1.7),

$$
f(e)=f(n \varphi(n))=f(e) D f(e)(\varphi(n)) o(n \varphi(n)) .
$$

Setting $L=D f(e)$,

$$
d(\varphi(n))=d\left(L_{\mid H}^{-1}(o(n \varphi(n)))\right) \leq \varepsilon(d(n)+d(\varphi(n))),
$$

for $n$ suitably close to $e$ and small $\varepsilon>0$, arbitrarily fixed. From this, we easily conclude that $\varphi$ is differentiable at $e_{N}$ and $D \varphi\left(e_{N}\right)$ is the null $h$-homomorphism. Now, we arbitrarily fix $R>0$ and consider sufficiently small $\lambda>0$, such that

$$
D_{R} \cap \delta_{1 / \lambda} S=D_{R} \cap\left\{\delta_{1 / \lambda} n \delta_{1 / \lambda} \varphi(n) \mid n \in D_{r}^{N}\right\} .
$$


Let $\left(\lambda_{k}\right)$ be a sequence of positive numbers converging to zero. Using [1, Proposition 4.5.5], we have to prove the following conditions:

(i) if $x=\lim _{k \rightarrow \infty} x_{k}$ for some sequence $\left(x_{k}\right)$ such that $x_{k} \in D_{R} \cap \delta_{1 / \lambda_{k}} S$, then we have $x \in D_{R} \cap N$;

(ii) if $x \in D_{R} \cap N$, then there exists a sequence ( $\left.x_{k}\right)$ such that $x_{k} \in D_{R} \cap \delta_{1 / \lambda_{k}} S$ and $x_{k} \rightarrow x$.

To prove (i), we write $x_{k}=\left(\delta_{1 / \lambda_{k}} n_{k}\right)\left(\delta_{1 / \lambda_{k}} \varphi\left(n_{k}\right)\right)$ and use Proposition 7.6 with respect to our complementary subgroups $N$ and $H$. Through the global diffeomorphism $\phi$ in (7.1), convergence of the sequence $\left(x_{k}\right)$ implies convergence of both $\left(\delta_{1 / \lambda_{k}} n_{k}\right)$ and $\left(\delta_{1 / \lambda_{k}} \varphi\left(n_{k}\right)\right)$. We notice that the limit $n_{0}$ of $\left(\delta_{1 / \lambda_{k}} n_{k}\right)$ belongs to $N$. Differentiability of $\varphi$ at $e_{N}$ gives

$$
\lim _{k \rightarrow \infty} \delta_{1 / \lambda_{k}} \varphi\left(\delta_{\lambda_{k}} n_{k}^{\prime}\right)=D \varphi\left(e_{N}\right)\left(n_{0}\right)=e_{H},
$$

where we have set $\delta_{1 / \lambda_{k}} n_{k}=n_{k}^{\prime}$. Then $x_{k} \rightarrow x=n_{0} \in D_{R} \cap N$ and our claim is achieved. Now we choose $x \in N \cap D_{R}$ and consider a sequence $\left(x_{l}\right)$ contained in $N \cap B_{R}$ converging to $x$. We observe that for every fixed $l$, there exists a sufficiently large $k_{l}$ such that $\delta_{\lambda_{k_{l}}} x_{l} \in D_{r}^{N}$ and

$$
\delta_{1 / \lambda_{k_{l}}}\left(\delta_{\lambda_{k_{l}}} x_{l} \varphi\left(\delta_{\lambda_{k_{l}}} x_{l}\right)\right) \in B_{R} \cap \delta_{1 / \lambda_{k}} S
$$

since differentiability of $\varphi$ at $e_{N}$ implies that

$$
\delta_{1 / \lambda_{k}}\left(\delta_{\lambda_{k}} x_{l} \varphi\left(\delta_{\lambda_{k}} x_{l}\right)\right) \longrightarrow x_{l} \quad \text { as } k \rightarrow \infty .
$$

The fact that $D \varphi\left(e_{N}\right)$ is the null $h$-homomorphism also implies that

$$
\delta_{1 / \lambda_{k_{l}}}\left(\delta_{\lambda_{k_{l}}} x_{l} \varphi\left(\delta_{\lambda_{k_{l}}} x_{l}\right)\right) \longrightarrow x D \varphi\left(e_{N}\right)(x)=x \quad \text { as } l \rightarrow \infty .
$$

Finally, we notice that (i) and (ii) exactly prove the two opposite inclusions between $N$ and $\operatorname{Tan}(S, e)$. This concludes the proof of the first part of Theorem 1.7.

Now, we keep the same notation used in the proof of Theorem 1.5 and observe that it is not restrictive to assume that $f(\bar{x})=e_{\mathbb{M}}$. In fact, in the general case it suffices to replace $f$ with the left translated $x \rightarrow f(\bar{x})^{-1} f(x)$, for which same assumptions hold. We know that the mapping $p \circ f_{\mid V}$ is invertible with differentiable inverse $\psi: W \longrightarrow V$ and $g(h)=f \circ \psi(h)=h \varphi(h)$ for every $h \in W$. By Proposition 2.10, we have that $D g\left(e_{H}\right): H \hookrightarrow \mathbb{M}$ identically embeds $H$ into $\mathbb{M}$. Then

$$
g(h)=h o(h)=h \varphi(h),
$$

so that $\varphi(h)=o(h)$. This implies that $D \varphi\left(e_{H}\right)$ exists and it is constantly equal to the unit element $e_{N} \in N$. To show the remaining claims, one can argue exactly as in the first part of this proof, using the fact that $\varphi$ is differentiable at $e_{H}$ with null differential at this point and replacing $N$ with $H$.

Corollary 10.6. Let $S$ be a $(\mathbb{G}, \mathbb{M})$-regular set of $\mathbb{G}$. Then for every $x \in S$,

$$
\mathcal{H}-\operatorname{dim}(\operatorname{Tan}(S, x))=\mathcal{H}-\operatorname{dim}(\mathbb{G})-\mathcal{H}-\operatorname{dim}(\mathbb{M}) .
$$


Proof. By Theorem 1.7, $\operatorname{Tan}(S, x)$ is $h$-isomorphic to $N=\operatorname{ker} D f(x)$, where $f$ is the continuously differentiable mapping defining $S$ around $x$ as a level set. Due to Proposition 7.10, the factorizing property of $h$-epimorphisms yields a complementary subgroup $H$ that is $h$-isomorphic to $\mathbb{M}$. By (7.2), our claim follows.

ExAmple 10.7. The fact that homogeneous tangent cones to a $(\mathbb{G}, \mathbb{M})$-regular set of $\mathbb{G}$ have a fixed Hausdorff dimension does not mean that they are all algebraically $h$ isomorphic. Let us consider the mapping

$$
f: \mathbb{H}^{2} \longrightarrow \mathbb{R}^{2}, \quad\left(x_{1}, x_{2}, x_{3}, x_{4}, x_{5}\right) \longrightarrow\left(\sqrt{x_{2}^{2}+x_{3}^{2}}, x_{4}\right),
$$

where $p=\exp \left(\sum_{j=1}^{5} x_{j} X_{j}\right) \in \mathbb{H}^{2}$ and $\left[X_{1}, X_{2}\right]=\left[X_{3}, X_{4}\right]=X_{5}$ are the nontrivial bracket relations of $\mathbb{H}^{2}$. The differential $D f(x)$ is represented by the matrix

$$
\left(\begin{array}{ccccc}
0 & \frac{x_{2}}{\sqrt{x_{2}^{2}+x_{3}^{2}}} & \frac{x_{3}}{\sqrt{x_{2}^{2}+x_{3}^{2}}} & 0 & 0 \\
0 & 0 & 0 & 1 & 0
\end{array}\right) .
$$

Then, defining the connected open set

$$
\Omega=\left\{\left(x_{1}, x_{2}, x_{3}, x_{4}, x_{5}\right) \in \mathbb{H}^{2} \mid x_{4}>0, x_{2}^{2}+x_{3}^{2}>0\right\},
$$

we notice that $S=\Omega \cap f^{-1}((1,1))$ is $\left(\mathbb{H}^{2}, \mathbb{R}^{2}\right)$-regular in $\mathbb{H}^{2}$. Now we fix $\xi=$ $(0,1,0,1,0)$ and $\eta=(0,0,1,1,0)$, observing that $\xi, \eta \in S$. Thus, it is easy to check that $\operatorname{Tan}(S, \xi)$ is a commutative graded group and $\operatorname{Tan}(S, \eta)$ is $h$-isomorphic to $\mathbb{H}^{1}$.

\section{Factorizing groups}

In this section we investigate the algebraic conditions under which either surjective or injective $h$-homomorphisms are $h$-monomorphisms or $h$-epimorphisms, respectively. Let $\mathbb{G}$ and $\mathbb{M}$ be arbitrary graded groups with algebras $\mathcal{G}$ and $\mathcal{M}$.

Definition 11.1 ( $h$-quotients and $h$-embeddings). We say that $\mathbb{M}$ is an $h$-quotient of $\mathbb{G}$ if there exists a normal homogeneous subgroup $N \subset \mathbb{G}$ such that $\mathbb{G} / N$ is $h$-isomorphic to $\mathbb{M}$. Analogously, $\mathbb{G} h$-embeds into $\mathbb{M}$ if and only if there exists a homogeneous subgroup $H$ of $\mathbb{M}$ which is $h$-isomorphic to $\mathbb{G}$.

Proposition 11.2. There is a normal homogeneous subgroup $N$ of $\mathbb{G}$ such that $\mathbb{G} / N$ is h-isomorphic to $\mathbb{M}$ if and only if there exists a surjective h-homomorphism $L: \mathbb{G} \longrightarrow \mathbb{M}$.

Proof. If we have a normal homogeneous subgroup $N$ such that there exists an $h$-isomorphism $T: \mathbb{G} / N \longrightarrow \mathbb{M}$, by Proposition 8.2 the canonical projection $\pi: \mathbb{G} \longrightarrow$ $\mathbb{G} / N$ is an $h$-homomorphism, and hence defining

$$
L=T \circ \pi: \mathbb{G} \longrightarrow \mathbb{M},
$$

we have obtained a surjective $h$-homomorphism. The converse is trivial. 
Remark. The previous proposition shows that $\mathbb{M}$ is an $h$-quotient of $\mathbb{G}$ if and only if there exists a surjective $h$-homomorphism $L: \mathbb{G} \longrightarrow \mathbb{M}$. In view of Proposition 8.2, $\mathbb{M}$ is stratified when $\mathbb{G}$ is stratified. It is also easy to check that $\mathbb{G} h$-embeds into $\mathbb{M}$ if and only if there exists an injective $h$-homomorphism $T: \mathbb{G} \longrightarrow \mathbb{M}$.

LEMMA 11.3. If $m$ is the dimension of the first layer of $\mathcal{G}$, then $\mathbb{R}^{k}$ is an h-quotient of $\mathbb{G}$ if and only if $k \leq m$.

Proof. Let $V_{1} \oplus V_{2} \oplus \cdots \oplus V_{\iota}$ be the direct decomposition of $\mathcal{G}$ into its layers. Let $\mathfrak{u}$ be an $(m-k)$-dimensional subspace of $V_{1}$. Then $\mathcal{N}=\mathfrak{u} \oplus V_{2} \oplus \cdots \oplus V_{\iota}$ is an ideal and clearly $\mathcal{G} / \mathcal{N}$ is $h$-isomorphic to $\mathbb{R}^{k}$. By Proposition $8.1, \mathbb{G} / N$ is also $h$-isomorphic to $\mathbb{R}^{k}$, where $N=\exp \mathcal{N}$. Conversely, assume that $\mathbb{R}^{k}$ is an $h$-quotient of some G. From Proposition 8.2, it follows that there exists $N$, corresponding to the ideal $\mathcal{N}=\mathcal{N}_{1} \oplus \cdots \oplus \mathcal{N}_{\iota}$ such that $\mathbb{R}^{k}$ is linearly isomorphic to $V_{1} / \mathcal{N}_{1}$, so $k \leq \operatorname{dim} V_{1}=m$.

EXAMPLE 11.4. The Heisenberg group $\mathbb{H}^{k}$ is not an $h$-quotient of $\mathbb{H}^{n}$, whenever $n>k$. By contradiction, assume that there exists a normal subgroup $N=\exp \pi$ such that $\mathbb{H}^{n} / N$ is $h$-isomorphic to $\mathbb{H}^{k}$. In terms of algebras, we have a $2(n-k)$-dimensional ideal $\pi$ of $\mathfrak{h}^{n}$ such that $\mathfrak{h}^{n} / \mathfrak{h}$ is $h$-isomorphic to $\mathfrak{h}^{k}$. This implies that there exists $\mathfrak{n}+Z^{\prime} \in \mathfrak{h}^{n} / \mathfrak{n}$ that corresponds to a nonvanishing element of the second layer of $\mathfrak{h}^{k}$. It follows that $\mathfrak{n} \subset \mathfrak{v}$ and $[\mathfrak{r}, \mathfrak{n}]=\{0\}$, since $\mathfrak{v}$ is the first layer of $\mathfrak{h}^{n}$. If we pick an element $X \in \mathfrak{v}$ such that $[X, \mathfrak{\pi}] \neq\{0\}$, then we meet a contradiction. In fact, $X \notin n$, since $\mathfrak{n}$ is commutative and this conflicts with the fact that $n$ is an ideal.

REMARK. In view of the previous remark, Example 11.4 shows the nonexistence of surjective $h$-homomorphisms from $\mathbb{H}^{n}$ to $\mathbb{H}^{k}$ whenever $n>k$, as it was proved in [17, Theorem 2.8], by explicit representation of $h$-homomorphisms from $\mathbb{H}^{n}$ to $\mathbb{H}^{k}$.

Definition 11.5 (Factorizing group as a quotient). We say that $\mathbb{M}$ factorizes $\mathbb{G}$ as a quotient if it is an $h$-quotient of $\mathbb{G}$ and every normal subgroup $N$ of $\mathbb{G}$ such that $\mathbb{G} / N$ is $h$-isomorphic to $\mathbb{M}$ has a complementary subgroup $H$.

Definition 11.6 (Factorizing group as a subgroup). We say that $\mathbb{G}$ factorizes $\mathbb{M}$ as $a$ subgroup if it $h$-embeds into $\mathbb{M}$ and every subgroup $H$ of $\mathbb{M}$ which is $h$-isomorphic to $\mathbb{G}$ has a complementary normal subgroup $N$.

As a corollary to Propositions 7.10 and 7.11, we have the following proposition.

Proposition 11.7. We have that $\mathbb{M}$ factorizes $\mathbb{G}$ as a quotient if and only if every surjective h-homomorphism is an h-epimorphism, and $\mathbb{G}$ factorizes $\mathbb{M}$ as a subgroup if and only if every injective h-homomorphism is an h-monomorphism.

Proposition 11.8. If $\mathbb{R}^{k}$ h-embeds into $\mathbb{G}$, then it factorizes $\mathbb{G}$ as a subgroup.

Proof. By hypothesis, the class of $k$-dimensional subgroups $H=\exp \mathfrak{h}$ of $\mathbb{G}$ which are $h$-isomorphic to $\mathbb{R}^{k}$ is nonempty and it corresponds to the $k$-dimensional commutative subgroups contained in $\exp V_{1}$. Let $\mathcal{N}_{1}$ be a subspace of $V_{1}$ such that $\mathfrak{h} \oplus \mathcal{N}_{1}=V_{1}$. Then $\mathcal{N}=\mathcal{N}_{1} \oplus V_{2} \oplus \cdots \oplus V_{\iota}$ is an ideal and $N=\exp \mathcal{N}$ is complementary to $H$. 
ReMark. If $\mathbb{R}^{k} h$-embeds into $\mathbb{M}$, then every injective $h$-homomorphism from $\mathbb{R}^{k}$ to $\mathbb{M}$ is an $h$-monomorphism. This immdiately follows joining Propositions 11.7 and 11.8.

Definition 11.9 (Factorizing group). We say that $\mathbb{G}$ factorizes $\mathbb{M}$ if it factorizes $\mathbb{M}$ both as a subgroup and as a quotient.

REMARK. It might be interesting from an algebraic viewpoint to investigate whether factorizing groups as a quotient are also factorizing as a subgroup and vice versa.

The following proposition is straightforward.

Proposition 11.10. $\mathbb{R}$ factorizes every stratified group.

Example 11.11 . One can easily find $\mathbb{G}$ and $\mathbb{M}$ such that there exist $h$-epimorphisms $L: \mathbb{G} \longrightarrow \mathbb{M}$, although $\mathbb{M}$ does not factorize $\mathbb{G}$ as a quotient. In fact, let us consider the 2-step stratified group $\mathbb{G}$ with Lie algebra $\mathcal{G}=V_{1} \oplus V_{2}$, where

$$
V_{1}=\operatorname{span}\left\{X_{1}, X_{2}, X_{3}, X_{4}\right\}, \quad V_{2}=\operatorname{span}\left\{Z_{23}, Z_{24}, Z_{34}\right\},
$$

and the only nontrivial bracket relations are

$$
\left[X_{2}, X_{3}\right]=Z_{23}, \quad\left[X_{2}, X_{4}\right]=Z_{24} \quad \text { and } \quad\left[X_{3}, X_{4}\right]=Z_{34} .
$$

Let $L_{1}: \mathbb{G} \longrightarrow \mathbb{R}^{2}$ be the $h$-epimorphism defined by

$$
L_{1}\left(\exp \left(\sum_{i=1}^{4} x_{i} X_{i}+z_{23} Z_{23}+z_{24} Z_{24}+z_{34} Z_{34}\right)\right)=\left(x_{1}, x_{2}\right)
$$

and let $\operatorname{ker} L_{1}=\exp n=\exp \left(n_{1} \oplus n_{2}\right)=N$. We have $n_{2}=V_{2}$ and $n_{1}=\operatorname{span}\left\{X_{3}, X_{4}\right\}$, so $H=\exp \left\{X_{1}, X_{2}\right\}$ is complementary to $N$, which easily follows from Proposition 7.6. However, if we consider the $h$-epimorphism $L_{2}: \mathbb{G} \longrightarrow \mathbb{R}^{2}$ defined by

$$
L_{2}\left(\exp \left(\sum_{i=1}^{4} x_{i} X_{i}+z_{23} Z_{23}+z_{24} Z_{24}+z_{34} Z_{34}\right)\right)=\left(x_{3}, x_{4}\right),
$$

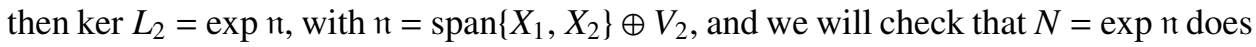
not admit any complementary subgroup. By contradiction, if $H$ is complementary to $N$, then Proposition 7.10 shows that the restriction $T: H \longrightarrow \mathbb{R}^{2}$ of $L_{2}$ is a group isomorphism. In particular, $H$ is commutative. In addition, Proposition 7.6 shows that $\mathfrak{h} \oplus \mathfrak{n}=\mathcal{G}$, where $\mathfrak{h}$ denotes the Lie algebra of $H$. Then $\mathfrak{h}$ is a two-dimensional commutative subalgebra of $\mathcal{G}$. We consider a basis $(v, w)$ of $\mathfrak{h}$, given by

$$
v=\sum_{j=1}^{4} \alpha_{j} X_{j}+Z \quad \text { and } \quad w=\sum_{j=1}^{4} \beta_{j} X_{j}+T,
$$

where $T, Z \in V_{2}$. The decomposition $\mathfrak{h} \oplus \mathfrak{n}=\mathcal{G}$ implies that the ordered set of vectors $\left(v, w, X_{1}, X_{2}, Z_{23}, Z_{24}, Z_{34}\right)$ is a basis of $\mathcal{G}$, and hence we must have $\alpha_{3} \beta_{4}-$ $\beta_{3} \alpha_{4} \neq 0$. As a consequence, $[v, w]=\left(\alpha_{2} \beta_{3}-\alpha_{3} \beta_{2}\right) Z_{23}+\left(\alpha_{2} \beta_{4}-\alpha_{4} \beta_{2}\right) Z_{24}+\left(\alpha_{3} \beta_{4}-\right.$ $\left.\alpha_{4} \beta_{3}\right) Z_{34} \neq 0$. This conflicts with the fact that $\mathfrak{h}$ is commutative. 
11.1. Factorizations in some H-type groups. Our aim here is to present some factorizing properties of $\mathbb{R}^{k}$ in some important H-type groups. These groups were introduced in [12].

Definition 11.12. Let $\mathfrak{g}$ be a Lie algebra equipped with an inner product $\langle\cdot, \cdot\rangle$ and let $z$ be a nontrivial subspace of $\mathfrak{g}$ such that $[\mathfrak{g}, \mathfrak{\jmath}]=0$ and $[\mathfrak{g}, \mathfrak{g}] \subset \mathfrak{z}$. Let $\mathfrak{v}$ be the orthogonal space of $z$ and define $J: z \longrightarrow \operatorname{End}(\mathfrak{y})$ by the formula

$$
\left\langle J_{Z} X, Y\right\rangle=\langle Z,[X, Y]\rangle
$$

for every $Z \in z$ and $X, Y \in \mathfrak{v}$. If $J$ satisfies the condition $\left|J_{Z} X\right|=|Z||X|$, then we say that $\mathfrak{g}$ is an H-type algebra.

From the previous definition, it follows that

$$
\left[X, J_{Z} X\right]=|X|^{2} Z \quad \text { for every }(X, Z) \in \mathfrak{v} \times \jmath .
$$

Then $\mathfrak{g}=\mathfrak{v} \oplus \mathfrak{z}$ is a 2-step stratified algebra of first layer $\mathfrak{v}$ and second layer $\mathfrak{z}$. In the sequel, we will utilize another well-known formula,

$$
J_{Z} J_{W}+J_{W} J_{Z}=-2\langle Z, W\rangle \mathrm{Id}_{\mathfrak{v}} .
$$

ExAmple 11.13. Let $(X, Y, Z)$ be an orthonormal basis of the Heisenberg algebra $\mathfrak{h}^{1}$, where $[X, Y]=Z$. Then, setting $z=\operatorname{span}\{Z\}, \mathfrak{v}=\operatorname{span}\{X, Y\}$ and $J_{Z} X=Y, J_{Z} Y=-X$, extended by linearity, it follows that $J$ makes $\mathfrak{h}^{1}$ an H-type algebra. The higher dimensional Heisenberg algebras $\mathfrak{h}^{n}$ can be seen as direct product of the irreducible Heisenberg algebras isomorphic to $\mathfrak{h}^{1}$ as

$$
\mathfrak{h}^{n}=\mathfrak{v}_{1} \oplus \cdots \oplus \mathfrak{v}_{n} \oplus \mathfrak{\jmath}
$$

where $\left(X_{i}, Y_{i}\right)$ is an orthonormal basis of $\mathfrak{p}_{i}$ and $J_{Z} X_{i}=Y_{i}, J_{Z} Y_{i}=-X_{i}$. Here we notice that setting $\omega\left(U_{1}, U_{2}\right)=\left\langle J_{Z} U_{1}, U_{2}\right\rangle$, we define a symplectic form on the $2 n$-dimensional first layer $\mathfrak{v}=\mathfrak{v}_{1} \oplus \cdots \oplus \mathfrak{v}_{n}$.

EXAMPLE 11.14. It is easy to check that $\mathbb{R}^{2}$ does not factorize $\mathfrak{h}^{1}$ as a quotient, although it is an $h$-quotient of $\mathfrak{h}^{1}$. In fact, the only one-dimensional ideal $\mathfrak{n}$ of $\mathfrak{h}^{1}$ is the second layer $z$ and we have already shown in Example 7.7 that there do not exist two-dimensional subalgebras complementary to $z$.

It is a standard exercise of symplectic geometry to notice that $\mathbb{R}^{k}$ factorizes the Heisenberg group $\mathbb{H}^{n}$ as a quotient, whenever $k \leq n$. In fact, one can consider the canonical symplectic structure induced on the first layer of the Heisenberg algebra, see, for instance, $[7,22]$.

Proposition 11.15. If $1 \leq k \leq n$, then $\mathbb{R}^{k}$ factorizes $\mathbb{H}^{n}$.

Remark. Joining Propositions 11.7 and 11.10, it follows that every surjective $h$-homomorphism $L: \mathbb{G} \longrightarrow \mathbb{R}$ and every injective $h$-homomorphism $L: \mathbb{R} \longrightarrow \mathbb{G}$ are 
an $h$-epimorphism and an $h$-monomoprhism, respectively. Joining Propositions 11.7 and 11.15 , for every $1 \leq k \leq n$, it follows that every surjective $h$-homomorphism $L$ : $\mathbb{H}^{n} \longrightarrow \mathbb{R}^{k}$ and every injective $h$-homomorphism $L: \mathbb{R}^{k} \longrightarrow \mathbb{H}^{n}$ are an $h$-epimorphism and an $h$-monomorphism, respectively. On the other hand, Example 11.14 shows that $\mathbb{R}^{k}$ is not an $h$-quotient of $\mathbb{H}^{n}$, whenever $k>n$.

Another example of an H-type group is the complexified Heisenberg group $\mathbb{H}_{2}^{1}$, where the center $\mathfrak{z}$ of its Lie algebra $\mathfrak{h}_{2}^{1}$ is two-dimensional and the first layer $\mathfrak{v}$ has dimension four. More information on this group can be found in [25].

Lemma 11.16. Let $\mathfrak{h}_{2}^{1}=\mathfrak{v} \oplus \mathfrak{z}$ be the six-dimensional real Lie algebra of the complexified Heisenberg group $\mathbb{H}_{2}^{1}$ and let $X \in \mathfrak{v}$ with $|X|=1$. Let $\left(Z_{1}, Z_{2}\right)$ be an orthonormal basis of 3 . Then the vectors

$$
R_{0}=X, \quad R_{1}=J_{Z_{1}} X, \quad R_{2}=J_{Z_{2}} X, \quad R_{3}=J_{Z_{1}} J_{Z_{2}} X
$$

form an orthonormal basis of $\mathfrak{v}$ and the only nontrivial bracket relations are given by

$$
\left[R_{0}, R_{1}\right]=\left[R_{2}, R_{3}\right]=Z_{1}, \quad\left[R_{0}, R_{2}\right]=-\left[R_{1}, R_{3}\right]=Z_{2} .
$$

Proof. Using only the properties of $J$ and the formula $\left[Y, J_{Z} Y\right]=|Y|^{2} Z$ for every $Y \in \mathfrak{v}$ and $Z \in \jmath$, it is easy to check that the $R_{i}$ form an orthonormal basis of $\mathfrak{v}$. The previous formula also yields $\left[R_{0}, R_{1}\right]=Z_{1}$ and $\left[R_{0}, R_{2}\right]=Z_{2}$. In addition,

$$
\begin{gathered}
\left\langle Z_{1},\left[R_{1}, R_{2}\right]\right\rangle=\left\langle J_{Z_{1}}^{2} X, J_{Z_{2}} X\right\rangle=-\left\langle X, J_{Z_{2}} X\right\rangle=0, \\
\left\langle Z_{2},\left[R_{1}, R_{2}\right]\right\rangle=\left\langle J_{Z_{2}} J_{Z_{1}} X, J_{Z_{2}} X\right\rangle=\left\langle J_{Z_{1}} X, X\right\rangle=0,
\end{gathered}
$$

so $\left[R_{1}, R_{2}\right]=0$. To prove that $\left[R_{0}, R_{3}\right]=0,\left[R_{1}, R_{3}\right]=-Z_{2}$ and $\left[R_{2}, R_{3}\right]=Z_{1}$, one argues in the same way, using additionally (11.2).

Proposition 11.17. $\mathbb{R}^{2}$ factorizes the complexified Heisenberg group $\mathbb{H}_{2}^{1}$ as a quotient.

Proof. Lemma 11.3 ensures that $\mathbb{R}^{2}$ is an $h$-quotient of $\mathbb{H}_{2}^{1}$. Let $N$ be the kernel of an $h$-epimorphism $L: \mathbb{H}_{2}^{1} \longrightarrow \mathbb{R}^{2}$ and set $\mathfrak{n}=\exp ^{-1}(N)=\mathfrak{n}_{1} \oplus \mathfrak{n}_{2}$, with $\mathfrak{n}_{1}=\mathfrak{n} \cap V_{1}$ and $\mathfrak{n}_{2}=\mathfrak{n} \cap V_{2}$, due to Proposition 7.2. Clearly, $\mathfrak{n}_{2}=\mathfrak{z}$ and $\operatorname{dim}\left(\mathfrak{n}_{1}\right)=2$. We have either $\operatorname{dim}\left(\left[\mathfrak{n}_{1}, \mathfrak{n}_{1}\right]\right)=0$ or $\operatorname{dim}\left(\left[\mathfrak{n}_{1}, \mathfrak{n}_{1}\right]\right)=1$. In the first case we choose an orthonormal basis $(X, Y)$ of $\mathfrak{n}_{1}$ and represent $Y$ as a linear combination of $R_{i}$, according to (11.3). Then the fact that $(X, Y)$ is an orthonormal basis and $[X, Y]=0$ imply that $Y=J_{Z_{1}} J_{Z_{2}} X$ for a fixed orthonormal basis $\left(Z_{1}, Z_{2}\right)$ of the center 3 . Now we simply notice that the commutative subalgebra $\mathfrak{h}=\operatorname{span}\left\{J_{Z_{1}} X, J_{Z_{2}} X\right\}$ satisfies $\mathfrak{h} \oplus \mathfrak{n}=\mathfrak{h}_{2}^{1}$. Let us consider the remaining case and take an orthonormal basis $(X, Y)$ of $n_{1}$. We have $[X, Y]=Z \neq 0$. Let $\left(T_{1}, T_{2}\right)$ be an orthonormal basis of $z$ such that $T_{1}=Z /|Z|$. Replacing $\left(Z_{1}, Z_{2}\right)$ in Lemma 11.16 with the orthonormal basis $\left(T_{1}, T_{2}\right)$, we get $Y=\alpha_{1} J_{T_{1}} X+\alpha_{3} J_{T_{1}} J_{T_{2}} X$, with $\left|\alpha_{1}\right|=|Z|>0$. By direct computation, one can check that the commutative subalgebra $\mathfrak{h}=\operatorname{span}\left\{X-\lambda J_{T_{2}} X, \lambda J_{T_{1}} X+J_{T_{1}} J_{T_{2}} X\right\}$ satisfies the condition $\mathfrak{h} \oplus \mathfrak{n}=\mathfrak{h}_{2}^{1}$ if we fix $\lambda \neq 0$ and $\lambda^{-1} \neq \alpha_{3} / \alpha_{1}$. This concludes the proof. 
Proposition 11.18. Let $\mathfrak{h}_{2}^{1}=\mathfrak{v} \oplus \mathfrak{z}$ be the complexified Heisenberg algebra. Then there do not exist commutative subalgebras of $\mathfrak{v}$ with dimension greater than two.

Proof. By the general formula (11.1), for every $X \in \mathfrak{v} \backslash\{0\}$, the mapping

$$
\operatorname{ad} \mathrm{X}: \mathfrak{v} \longrightarrow \mathfrak{z}, \quad Y \longrightarrow[X, Y]
$$

is surjective, so its kernel is two-dimensional. By contradiction, the existence of a commutative subalgebra of $\mathfrak{v}$ with dimension greater than two would conflict with the dimension of the kernel.

Corollary 11.19. For $k=3,4$ we have that $\mathbb{R}^{k}$ does not h-embed into $\mathbb{H}_{2}^{1}$ and does not factorize $\mathbb{H}_{2}^{1}$ as a quotient.

Proof. The first assertion immediately follows from both Definition 11.1 and Proposition 11.18. Concerning the proof of the second assertion, in view of Proposition 11.7 , we consider a surjective $h$-homomorphism $L: \mathfrak{h}_{2}^{1} \longrightarrow \mathbb{R}^{3}$. By contradiction, if $L$ is an $h$-epimorphism, then we get a three-dimensional subalgebra of $\mathfrak{v}$ that is $h$-isomorphic to $\mathbb{R}^{3}$. This conflicts with Proposition 11.18 and concludes the proof.

11.2. Factorizations in some free stratified groups. We denote by $\mathfrak{g}_{p, v}$ the free $v$-step stratified algebra on $p$ generators, and by $\mathbb{G}_{p, v}$ its simply connected Lie group.

REMARK. Let us show that $\mathfrak{g}_{p, v}$ is an $h$-quotient of $\mathfrak{g}_{r, v} \oplus \mathfrak{a}$, with $p \leq r$, and $\mathfrak{a}$ is a stratified algebra. Here $\oplus$ denotes the direct product of Lie algebras. Let $\mathcal{B}_{r, v}=\left\{\left[X_{j_{1}}, \ldots,\left[X_{j_{s-1}}, X_{j_{s}}\right] \ldots\right] \mid 1 \leq s \leq v,\left(j_{1}, \ldots, j_{s}\right) \in \mathcal{A}_{r, v}^{s} \subset\{1, \ldots, r\}^{s}\right\}$ and $\mathcal{B}_{p, v}^{\prime}=\left\{\left[X_{j_{1}}^{\prime}, \ldots,\left[X_{j_{s-1}}^{\prime}, X_{j_{s}}^{\prime}\right] \ldots\right] \mid 1 \leq s \leq v,\left(j_{1}, \ldots, j_{s}\right) \in \mathcal{A}_{p, v}^{\prime s} \subset\{1, \ldots, p\}^{s}\right\} \quad$ be bases of $\mathfrak{g}_{r, v}$ and $\mathfrak{g}_{p, v}$, respectively. Setting

$$
L\left(\left[X_{j_{1}},\left[X_{j_{2}}, \ldots,\left[X_{j_{s-1}}, X_{j_{s}}\right]\right] \ldots\right]\right):=\left[X_{j_{1}}^{\prime},\left[X_{j_{2}}^{\prime}, \ldots,\left[X_{j_{s-1}}^{\prime}, X_{j_{s}}^{\prime}\right]\right] \ldots\right],
$$

for every $1 \leq s \leq v$ and every $\left(j_{1}, \ldots, j_{s}\right) \in \mathcal{A}_{p, v}^{\prime s} \subset \mathcal{A}_{r, v}^{s}$,

$$
L\left(\left[X_{j_{1}},\left[X_{j_{2}}, \ldots,\left[X_{j_{s-1}}, X_{j_{s}}\right]\right] \ldots\right]\right):=0
$$

for every $1 \leq s \leq v$ and every $\left(j_{1}, \ldots, j_{s}\right) \in \mathcal{A}_{r, v}^{s} \backslash \mathcal{A}_{p, v}^{\prime s}$ and $L(\mathfrak{a})=\{0\}$. It is easy to check that $L: \mathfrak{g}_{r, v} \oplus \mathfrak{a} \longrightarrow \mathfrak{g}_{p, v}$ is a surjective $h$-homomorphism.

Proposition 11.20. Let $\mathbb{P}$ be a stratified group such that $\mathbb{G}_{p, v}$ is an h-quotient of $\mathbb{P}$. Then $\mathbb{G}_{p, v}$ factorizes $\mathbb{P}$ as a quotient.

Proof. We apply Proposition 11.7. By hypothesis, we have that $L: \mathcal{P} \longrightarrow \mathfrak{g}_{p, v}$ is a surjective $h$-homomorphism, where $\mathcal{P}=W_{1} \oplus \cdots \oplus W_{\iota}$ is the stratified algebra of $\mathbb{P}$. Let $X_{1}, \ldots, X_{p}$ be generators of $\mathfrak{g}_{p, v}$. Then there exist $U_{1}, \ldots, U_{p} \in W_{1}$ such that $L\left(U_{i}\right)=X_{i}$. By hypothesis, we consider the basis

$$
\mathcal{B}=\left\{\left[X_{j_{1}},\left[X_{j_{2}}, \ldots,\left[X_{j_{s-1}}, X_{j_{s}}\right]\right] \ldots\right]: 1 \leq s \leq v,\left(j_{1}, \ldots, j_{s}\right) \in \mathcal{A}^{s} \subset\{1, \ldots, p\}^{s}\right\}
$$


of $\mathfrak{g}_{p, v}$. The homomorphism property of $L$ implies that

$$
L\left(\left[U_{j_{1}},\left[U_{j_{2}}, \ldots,\left[U_{j_{s-1}}, U_{j_{s}}\right]\right] \ldots\right]\right)=\left[X_{j_{1}},\left[X_{j_{2}}, \ldots,\left[X_{j_{s-1}}, X_{j_{s}}\right]\right] \ldots\right],
$$

so the family

$$
\mathcal{B}^{\prime}=\left\{\left[U_{j_{1}},\left[U_{j_{2}}, \ldots,\left[U_{j_{s-1}}, U_{j_{s}}\right]\right] \ldots\right]: 1 \leq s \leq v,\left(j_{1}, \ldots, j_{s}\right) \in \mathcal{A}^{\prime s} \subset\{1, \ldots, p\}^{s}\right\}
$$

is a basis of $\mathfrak{h}=\operatorname{Lie}-\operatorname{span}\left\{U_{1}, \ldots, U_{p}\right\}$. As a consequence, we have that $L$ maps $\mathfrak{h}$ $h$-isomorphically onto $\mathfrak{g}_{p, v}$.

COROllary 11.21. $\mathbb{H}^{1}$ factorizes $\mathbb{G}_{r, 2} \times \mathbb{G}^{\prime}$ as a quotient for every $r \geq 2$.

Proof. Observe that $\mathfrak{h}^{1}=\mathfrak{g}_{2,2}$, take into account the first remark of Section 11.2 and apply Proposition 11.20.

The following corollaries are straightforward.

Corollary 11.22. $\mathbb{H}^{1}$ factorizes $\mathbb{G}_{r, 2} \times \mathbb{G}_{r, 2} \times \cdots \times \mathbb{G}_{r, 2}$ as a quotient for every $r \geq 2$.

Corollary 11.23. $\mathbb{H}^{1}$ factorizes $\mathbb{H}^{1} \times \mathbb{H}^{1} \times \cdots \times \mathbb{H}^{1}$ as a quotient.

REMARK. Although $\mathbb{H}^{1}$ factorizes $\mathbb{H}^{1} \times \mathbb{H}^{1} \times \cdots \times \mathbb{H}^{1}$ as a quotient, note that there are a few nontrivial $h$-epimorphisms between these groups. In fact, they are all of the form

$$
L: \overbrace{\mathbb{H}^{1} \times \mathbb{H}^{1} \times \cdots \times \mathbb{H}^{1}}^{n \text {-times }} \longrightarrow \mathbb{H}^{1}, \quad L\left(a_{1}, \ldots, a_{n}\right)=J\left(a_{k}\right),
$$

for some fixed $k \in\{1, \ldots, n\}$ and some $h$-isomorphism $J: \mathbb{H}^{1} \longrightarrow \mathbb{H}^{1}$.

\section{Examples of $(\mathbb{G}, \mathbb{M})$-regular sets}

Existence of different types of intrinsically regular sets in a given graded group $\mathbb{P}$ depends on the corresponding algebraic factorizations. In correspondence to the fact that $\mathbb{R}^{k}$ factorizes a graded group $\mathbb{M}$ as a subgroup, we have the following theorem.

Theorem 12.1. Let $\mathbb{M}$ be a graded group, let $n$ be the maximum over all dimensions of commutative subalgebras contained in the first layer and choose $1 \leq k \leq n$. Then every $k$-dimensional $C^{1}$ smooth submanifold is horizontal if and only if it is an $\left(\mathbb{R}^{k}, \mathbb{M}\right)$ regular set of $\mathbb{M}$.

PRoof. Every $k$-dimensional commutative subalgebra of the first layer also represents a trivial example of a $k$-dimensional horizontal submanifold. This shows, by definition of $n$, that this family is nonempty for every $1 \leq k \leq n$. Recall that a $k$-dimensional horizontal submanifold is locally parametrized by a $C^{1}$ contact mapping defined on an open subset of $\mathbb{R}^{k}$. In other words, it is locally parametrized by a continuously $h$-differentiable contact mapping with injective differential. Then Theorem 1.1 shows that this mapping is continuously differentiable. Furthermore, since in this case classical differentiability coincides with $h$-differentiability, then (1.1) 
shows that the classical differential corresponds to the differential. Then the differential of the local parametrization is an injective $h$-homomorphism. As a result, the third remark of Section 11 implies that the differential is an $h$-monomorphism. Taking into account Definition 10.3, we have shown that every $C^{1}$ smooth horizontal submanifold contained in $\mathbb{M}$ is an $\left(\mathbb{R}^{k}, \mathbb{M}\right)$ regular set of $\mathbb{M}$. Clearly, we have also proved that there do not exist $k$-dimensional horizontal submanifolds when $k>n$, since the injective differential of the local parametrization would imply the existence of a commutative subalgebra in the first layer of $\mathcal{M}$ with dimension greater than $k$, which is a contradiction. Now, let $S$ be an $\left(\mathbb{R}^{k}, \mathbb{M}\right)$-regular set, where clearly $1 \leq k \leq n$. Again Theorem 1.1 shows that continuously differentiable mappings on $\mathbb{R}^{k}$ exactly correspond to contact mappings of class $C^{1}$, so $S$ is a $C^{1}$ smooth horizontal submanifold.

Definition 12.2. We say that a homogeneous subgroup of $\mathbb{H}^{n}$ is horizontal if its Lie algebra is contained in the first layer $\mathfrak{v}$ and vertical if it contains the second layer $z$.

Remark. Notice that horizontal subgroups are always commutative, whereas homogeneous normal subgroups exactly characterize vertical subgroups.

Proposition 7.6 and elementary arguments give the following proposition.

Proposition 12.3. Every two complementary subgroups of $\mathbb{H}^{n}$ correspond to a horizontal subgroup and a vertical subgroup.

According to the next example, we may have H-type groups factorized by complementary subgroups, none of which is a normal subgroup.

ExAmple 12.4. Let us consider the complexified Heisenberg group $\mathbb{H}_{2}^{1}$ along with the bases $\left(R_{0}, R_{1}, R_{2}, R_{3}\right)$ and $\left(Z_{1}, Z_{2}\right)$, defined in Lemma 11.16. We define the homogeneous commutative subalgebras $\mathfrak{a}=\operatorname{span}\left\{R_{0}, R_{3}, Z_{1}\right\}$ and $\mathfrak{b}=\operatorname{span}\left\{R_{1}, R_{2}, Z_{2}\right\}$. Clearly, $\mathfrak{a} \oplus \mathfrak{b}=\mathfrak{h}_{2}^{1}$, and hence from Proposition 7.6 it follows that $A=\exp \mathfrak{a}$ and $B=\exp \mathfrak{b}$ are complementary subgroups of $\mathbb{H}_{2}^{1}$. On the other hand, $\left[R_{0}, R_{2}\right]=Z_{2} \notin \mathfrak{a}$ and $\left[R_{0}, R_{1}\right]=Z_{1} \notin \mathfrak{b}$ imply that both $\mathfrak{a}$ and $\mathfrak{b}$ are not ideals of $\mathfrak{h}_{2}^{1}$.

If we assume that one of the two complementary subgroups of $\mathbb{H}_{2}^{1}$ is normal, then the other has to be horizontal and commutative, as shown in the next proposition.

Proposition 12.5. Let $N$ and $H$ be complementary subgroups of $\mathbb{H}_{2}^{1}$, where $N$ is normal. Then $N$ contains the center of the group, top- $\operatorname{dim}(N)$ is either 4 or 5 , and $H$ is commutative and horizontal, that is, $H \subset \exp \mathfrak{v}$.

Proof. Let $N=\exp \mathfrak{n}$ and $H=\exp \mathfrak{h}$. If $\operatorname{dim}(\mathfrak{n}) \geq 3$, then $\mathfrak{n} \supset \mathfrak{z}$ by (11.1). Then in this case $\operatorname{dim}(\mathfrak{h}) \leq 3$ and $\mathfrak{h} \subset \mathfrak{v}$. By Proposition 11.18, it follows that $\operatorname{dim}(\mathfrak{h}) \leq 2$. Then the case $\operatorname{dim}(\mathfrak{n})=3$ cannot occur. If $\operatorname{dim}(\mathfrak{n}) \leq 2$, then $\mathfrak{n} \subset \mathfrak{z}$, and Proposition 11.18 prevents the existence of $\mathfrak{h} \subset \mathfrak{v}$, which should be commutative. As a result, the only allowed possibilities are $\operatorname{dim}(\mathfrak{})=4$ and $\operatorname{dim}(\mathfrak{n})=5$. 
Theorem 12.6. The families of $\left(\mathbb{H}_{2}^{1}, \mathbb{R}\right)$-regular sets, $\left(\mathbb{H}_{2}^{1}, \mathbb{R}^{2}\right)$-regular sets, $\left(\mathbb{R}^{2}, \mathbb{H}_{2}^{1}\right)$ regular sets and $\left(\mathbb{R}, \mathbb{H}_{2}^{1}\right)$-regular sets constitute all nontrivial intrinsically regular sets of $\mathbb{H}_{2}^{1}$.

Proof. By Propositions 7.10 and 12.5, we are allowed to consider only level sets defined through continuously differentiable mappings with values in $\mathbb{R}^{k}, k=1,2$, and defined on an open set of $\mathbb{H}_{2}^{1}$. Every $C^{1}$ mapping $f: \Omega \subset \mathbb{H}_{2}^{1} \longrightarrow \mathbb{R}^{2}$ with surjective differential defines an $\left(\mathbb{H}_{2}^{1}, \mathbb{R}^{2}\right)$-regular set, since it is continuously differentiable and its differential is an $h$-epimorphism due to Proposition 11.17. This allows us to apply Theorem 1.4. We argue in the same way for $C^{1}$ mappings $f: \Omega \subset \mathbb{H}_{2}^{1} \longrightarrow \mathbb{R}$, due to Proposition 11.10.

Analogously, Propositions 7.11 and 12.5 allow us to consider only image sets from open subsets of $\mathbb{R}^{k}, k=1,2$, with values in $\mathbb{H}_{2}^{1}$. By Theorem 12.1, $\left(\mathbb{R}, \mathbb{H}_{2}^{1}\right)$-regular sets and $\left(\mathbb{R}^{2}, \mathbb{H}_{2}^{1}\right)$-regular sets of $\mathbb{H}_{2}^{1}$ exist and correspond to one-dimensional and two-dimensional $C^{1}$ smooth horizontal submanifolds of $\mathbb{H}_{2}^{1}$.

\section{Acknowledgements}

I am grateful to Fulvio Ricci for his fruitful comments and pleasant discussions. I thank Yu. L. Sachkov for the interesting discussions we had on Lie groups theory when I was at ISAS in Trieste. I thank Raul Serapioni for his subtle observations on the issue of higher codimensional intrinsically regular level sets. I thank Alessandro Ottazzi for some inspiring discussions on possible developments for factorizations of stratified groups. I thank Francesco Serra Cassano for his comments on a preliminary version of this work.

\section{References}

[1] L. Ambrosio and P. Tilli, Selected Topics on Analysis in Metric Spaces (Oxford University Press, 2003).

[2] L. Capogna and M. Cowling, 'Conformality and Q-Harmonicity in Carnot groups', Duke Math. J. 135(3) (2006), 455-479.

[3] H. Federer, Geometric Measure Theory (Springer, 1969).

[4] G. B. Folland and E. M. Stein, Hardy Spaces on Homogeneous Groups (Princeton University Press, 1982).

[5] B. Franchi, R. Serapioni and F. Serra Cassano, 'Regular hypersurfaces, intrinsic perimeter and implicit function theorem in Carnot groups', Comm. Anal. Geom. 11(5) (2003), 909-944.

[6] B. Franchi, R. Serapioni and F. Serra Cassano, 'On the structure of finite perimeter sets in step 2 Carnot groups', J. Geom. Anal. 13(3) (2003), 421-466.

[7] B. Franchi, R. Serapioni and F. Serra Cassano, 'Regular submanifolds, graphs and area formula in Heisenberg groups', Adv. Math. 211(1) (2007), 152-203.

[8] M. Gromov, 'Carnot-Carathéodory spaces seen from within', in: Subriemannian Geometry, Progress in Mathematics, 144 (eds. A. Bellaiche and J. Risler) (Birkhäuser, Basel, 1996).

[9] P. Hajlasz and P. Koskela, 'Sobolev met Poincaré', Mem. Amer. Math. Soc. 145 (2000).

[10] W. Hebisch and A. Sikora, 'A smooth subadditive homogeneous norm on a homogeneous group', Studia Math. 96(3) (1990), 231-236.

[11] I. N. Herstein, Algebra, Editori riuniti (1994), translation from Topics in Algebra. 
[12] A. Kaplan, 'Fundamental solutions for a class of hypoelliptic PDE generated by composition of quadratic forms', Trans. Amer. Math. Soc. 258(1) (1980), 147-153.

[13] B. Kirchheim and F. Serra Cassano, 'Rectifiability and parametrization of intrinsic regular surfaces in the Heisenberg group', Ann. Sc. Norm. Super. Pisa Cl. Sci. (5) 3(4) (2004), 871-896.

[14] N. G. LLoyd, Degree Theory (Cambridge University Press, 1978).

[15] V. Magnani, 'Differentiability and area formula on stratified Lie groups', Houston J. Math. 27(2) (2001), 297-323.

[16] V. Magnani, 'Elements of geometric measure theory on sub-Riemannian groups', PhD Theses series of Scuola Normale Superiore, 2002.

[17] V. Magnani, 'On a general coarea inequality and applications', Ann. Acad. Sci. Fenn. Math. 27 (2002), 121-140.

[18] V. Magnani, 'The coarea formula for real-valued Lipschitz maps on stratified groups', Math. Nachr. 278(14) (2005), 1-17.

[19] V. Magnani, 'Characteristic points, rectifiability and perimeter measure on stratified groups', J. Eur. Math. Soc. 8(4) (2006), 585-609.

[20] V. Magnani, 'Pansu differentiability and intrinsic submanifolds in stratified groups', 2007, arXiv:math/0701322v1.

[21] V. Magnani, 'Contact equations, Lipschitz extensions and isoperimetric inequalities', Calc. Var. Partial Differential Equations 39(1-2) (2010), 233-271.

[22] D. McDuff and D. Salamon, Introduction to Symplectic Topology (Oxford University Press, 1995).

[23] P. Pansu, 'Croissance des boules et des géodésiques fermeés dans les nilvariété', Ergod. Theory Dynam. Sys. 3 (1983), 415-445.

[24] P. Pansu, 'Métriques de Carnot-Carathéodory quasiisométries des espaces symétriques de rang un', Ann. Math. 129 (1989), 1-60.

[25] H. M. Reimann and F. Ricci, 'The complexified Heisenberg group', in: Proceedings on Analysis and Geometry (in Russian) (Novosibirsk Akademgorodok, 1999) (Izdat. Ross. Akad. Nauk Sib. Otd. Inst. Mat, Novosibirsk, 2000), 465-480.

[26] J. T. Schwartz, 'Nonlinear functional analysis', in: Courant Institute of Mathematical Sciences (New York University, 1965).

[27] E. M. Stein, Harmonic Analysis (Princeton University Press, 1993).

[28] V. S. Varadarajan, Lie groups, Lie Algebras and their Representation (Springer, New York, 1984).

[29] B. Warhurst, 'Contact and Pansu differentiable maps on Carnot groups', Bull. Aust. Math. Soc. 77(3) (2008), 495-507.

[30] F. W. Warner, Foundations of Differentiable Manifolds and Lie Groups (Foresman and Company, London, 1971).

VALENTINO MAGNANI, Dipartimento di Matematica, Università di Pisa,

Largo Bruno Pontecorvo 5, I-56127, Pisa

e-mail:magnani@dm.unipi.it 